\title{
LOCAL NULL CONTROLLABILITY OF A TWO-DIMENSIONAL FLUID-STRUCTURE INTERACTION PROBLEM
}

\author{
Muriel Boulakia $^{1}$ And Axel Osses ${ }^{2}$
}

\begin{abstract}
In this paper, we prove a controllability result for a fluid-structure interaction problem. In dimension two, a rigid structure moves into an incompressible fluid governed by Navier-Stokes equations. The control acts on a fixed subset of the fluid domain. We prove that, for small initial data, this system is null controllable, that is, for a given $T>0$, the system can be driven at rest and the structure to its reference configuration at time $T$. To show this result, we first consider a linearized system. Thanks to an observability inequality obtained from a Carleman inequality, we prove an optimal controllability result with a regular control. Next, with the help of Kakutani's fixed point theorem and a regularity result, we pass to the nonlinear problem.
\end{abstract}

Mathematics Subject Classification. 35Q30, 93C20.

Received October 27, 2005. Revised April 5, 2006.

Published online July 20, 2007.

\section{INTRODUCTION AND MAIN RESULT}

\subsection{Introduction}

We consider a rigid structure immersed in a viscous incompressible fluid. At time $t$, the structure occupies the smooth connected domain $\Omega_{S}(t)$. The structure and the fluid are contained in a fixed bounded connected open set $\Omega \subset \mathbb{R}^{2}$ with a regular boundary. We suppose that $\Omega_{S}(0)$ and $\Omega$ have a smooth boundary (for instance $\mathcal{C}^{2}$ ). The time evolution of the fluid eulerian velocity $u$ is governed by the incompressible Navier-Stokes equations (for simplicity, we assume that the fluid density is constant and equal to 1):

$$
\left\{\begin{array}{l}
\left(\partial_{t} u+(u \cdot \nabla) u\right)(t, x)-\operatorname{div} \sigma(u, p)(t, x)=f(t, x) 1_{\omega}(x), \forall x \in \Omega_{F}(t), \forall t \in(0, T), \\
\operatorname{div} u(t, x)=0, \forall x \in \Omega_{F}(t), \forall t \in(0, T) .
\end{array}\right.
$$

For any $t \in(0, T)$, these equations are satisfied on $\Omega_{F}(t)=\Omega \backslash \overline{\Omega_{S}(t)}$, the fluid domain. The tensor $\sigma(u, p)$ is the Cauchy tensor given by

$$
\sigma(u, p)=2 \epsilon(u)-p \mathrm{Id},
$$

\footnotetext{
Keywords and phrases. Controllability, fluid-solid interaction, Navier-Stokes equations, Carleman estimates.

1 Laboratoire de Mathématiques Appliquées, Université de Versailles-St-Quentin, 45 avenue des États-Unis, 78035 Versailles Cedex, France; boulakia@math.uvsq.fr

2 Departamento de Ingenería Matemática and Centro de Modelamiento Matemático UMI 2807 CNRS, Facultad de Ciencias de Físicas y Matemáticas, Universidad de Chile, Casilla 170/3 - Correo 3, Santiago, Chile; axosses@dim.uchile.cl

(c) EDP Sciences, SMAI 2007
} 
where $\epsilon(u)=\frac{1}{2}\left(\nabla u+\nabla u^{t}\right)$ is the symmetric part of the gradient. Here, $p$ is the pressure of the fluid. Without lost of generality, we have supposed that the viscosity is equal to 1 . Finally $f$ is the control function which acts over a fixed small nonempty open subset $\omega$ of the fluid domain $\Omega_{F}(t)$ and $1_{\omega}$ is the characteristic function of the domain $\omega$.

The motion of the structure is given by the translation velocity which is the velocity of the center of mass of the structure $a(t) \in \mathbb{R}^{2}$ and by the instantaneous rotation velocity denoted $r(t) \in \mathbb{R}$. The equations of the structure motion are given by the balance of linear and angular momentum. So, without the action of external forces, we have, for all $t \in(0, T)$

$$
\begin{aligned}
& m \ddot{a}(t)=\int_{\partial \Omega_{S}(t)} \sigma(u, p) n \mathrm{~d} \sigma(x), \\
& J \dot{r}(t)=\int_{\partial \Omega_{S}(t)}(\sigma(u, p) n) \cdot(x-a(t))^{\perp} \mathrm{d} \sigma(x) .
\end{aligned}
$$

We have denoted by $m>0$ the mass of the rigid structure and $J>0$ its moment of inertia. Moreover, $x^{\perp}$ is defined by

$$
\forall x=\left(x_{1}, x_{2}\right) \in \mathbb{R}^{2}, x^{\perp}=\left(-x_{2}, x_{1}\right) .
$$

At last, $n$ is the outward unit normal to $\partial \Omega_{S}(t)$. On the interface, we consider a non-slip boundary condition. Therefore, we have, for all $t \in(0, T)$

$$
\begin{aligned}
& u(t, x)=0, \forall x \in \partial \Omega, \\
& u(t, x)=\dot{a}(t)+r(t)(x-a(t))^{\perp}, \forall x \in \partial \Omega_{S}(t) .
\end{aligned}
$$

We define up to a constant the angle $\theta$ associated to the rotation velocity

$$
r=\dot{\theta} .
$$

The system is completed by the following initial conditions:

$$
u(0, \cdot)=u_{0} \text { in } \Omega_{F}(0), a(0)=a_{0}, \dot{a}(0)=a_{1}, \theta(0)=\theta_{0}, r(0)=r_{0},
$$

where $a_{0} \in \mathbb{R}^{2}$ the center of mass at initial time, $\theta_{0} \in \mathbb{R}, u_{0} \in H^{3}\left(\Omega_{F}(0)\right)^{2}, a_{1} \in \mathbb{R}^{2}$ and $r_{0} \in \mathbb{R}$ satisfy

$$
\operatorname{div} u_{0}=0 \text { in } \Omega_{F}(0), u_{0}=a_{1}+r_{0}\left(x-a_{0}\right)^{\perp} \text { on } \partial \Omega_{S}(0) \text { and } u_{0}=0 \text { on } \partial \Omega .
$$

At time $t$, the domain occupied by the structure $\Omega_{S}(t)$ is defined by

$$
\Omega_{S}(t)=X\left(t, \Omega_{S}(0)\right),
$$

where $X$ denotes the flow associated to the motion of the structure:

$$
X(t, y)=a(t)+R_{\theta(t)-\theta_{0}}\left(y-a_{0}\right), \forall y \in \Omega_{S}(0), \forall t \in(0, T) .
$$

Here, $R_{\theta}$ is the rotation matrix of angle $\theta$. We have chosen to denote by $y$ the lagrangian coordinate and by $x$ the eulerian coordinate. We can also notice that equation (1.5) allows to extend $u$ on the whole domain $\Omega$. We still denote $u$ the global velocity defined on the solid domain by

$$
u(t, x)=\dot{a}(t)+r(t)(x-a(t))^{\perp}, \forall x \in \Omega_{S}(t), \forall t \in(0, T) .
$$

We also extend $u_{0}$ on $\Omega$ in the same way. Thus, if we define

$$
V=\left\{v \in H_{0}^{1}(\Omega) / \operatorname{div} v=0 \text { in } \Omega\right\},
$$


then, for a.e. $t$ in $(0, T), u(t)$ belongs to $V$.

This problem satisfies an a priori estimate. Indeed, if we denote $E$ the global energy:

$$
E(t)=\frac{1}{2} \int_{\Omega_{F}(t)}|u(t, x)|^{2} \mathrm{~d} x+\frac{m}{2}|\dot{a}(t)|^{2}+\frac{J}{2}|r(t)|^{2}+\int_{0}^{t} \int_{\Omega_{F}\left(t^{\prime}\right)}\left|\nabla u\left(t^{\prime}, x\right)\right|^{2} \mathrm{~d} x \mathrm{~d} t^{\prime},
$$

we have

$$
E(t) \leq E(0)+C(T) \int_{0}^{t} \int_{\omega}\left|f\left(t^{\prime}, x\right)\right|^{2} \mathrm{~d} x \mathrm{~d} t^{\prime}
$$

Let us mention that [3] and [5] prove the existence of local solutions for this model (see also the references therein). In [18], a global existence result is proven: in particular, weak solutions of the fluid-structure problem are defined beyond collisions. Moreover, [19] obtains a regularity result valid as long as no collisions occur. In our study, we will need to keep this non-collision condition. We also want to avoid contact between the structure and the control domain. We consider an initial position such that

$$
\Omega_{S}(0) \subset \Omega \backslash \omega, \mathrm{d}\left(\overline{\Omega_{S}(0)}, \partial(\Omega \backslash \omega)\right)>0, \int_{\partial \Omega_{S}(0)}\left(y-a_{0}\right) \mathrm{d} \sigma(y)=0 .
$$

The last hypothesis will be necessary to obtain the Carleman inequality given in subsection 1.5. Indeed, thanks to this hypothesis, we will be able to deduce estimates for the structure velocity from estimates on the interface of the fluid velocity. It will come from the fact that, if $u=\dot{a}+r(x-a)^{\perp}$ on $\partial \Omega_{S}(t)$, we have

$$
\int_{\partial \Omega_{S}(t)}|u|^{2}=|\dot{a}|^{2} \int_{\partial \Omega_{S}(t)} 1+|r|^{2} \int_{\partial \Omega_{S}(t)}|x-a|^{2}=|\dot{a}|^{2} \int_{\partial \Omega_{S}(0)} 1+|r|^{2} \int_{\partial \Omega_{S}(0)}\left|y-a_{0}\right|^{2},
$$

thanks to the last hypothesis of (1.9). This hypothesis will be satisfied for a ball, an ellipse and more generally for any structure symmetric with respect to the center of mass.

In this paper, we will be concerned with the null controllability of the system presented above. In [6], the local null controllability is proved in dimension one for a particle evolving in a fluid modeled by Burgers equation. This one-dimensional model has been analyzed in [21] and in [22]. Simplified problems for the interaction between an elastic structure and a fluid are studied in $[16,17,23]$. The controllability of Navier-Stokes equations is the subject of recent works. The methods used to deal with Navier-Stokes equations in our fluid-structure problem are essentially due to papers $[10,12]$.

Our article has been announced in a preprint [2]. Let us mention that a simultaneous and independent work has been achieved in [14]. Some differences can be emphasized. Indeed, in this paper, the geometry of the rigid solid is necessarily a ball while, in our paper, it only has to satisfy some symmetric hypothesis. The methods used in [14] and in our work are different even if, in the two works, the main tool is a Carleman inequality. In particular, in [14], the nonlinear problem is not proved with a compactness argument and thus initial conditions are not as regular as in our work.

Remark 1. In (1.9), we only assume that no contact occurs between the structure and the global boundary at initial time. As we will see, we keep this non-collision condition for all $t \in(0, T)$. Indeed, if initial data are small, then the control function is also small (see Prop. 6) and thus the displacement of the structure stays small. Thus, if initial data are small enough, we then get that

$$
\mathrm{d}\left(\overline{\Omega_{S}(t)}, \partial(\Omega \backslash \omega)\right)>0
$$


To conclude this subsection, we introduce function spaces on moving domains. In the following, for the sake of readability, we omit to indicate with respect to which variable we are integrating, except when this is not obvious.

Definition 1. We consider a domain $S \subset \Omega$ and, for each $t$, the domain $S(t)=\Psi(t, S) \subset \Omega$ where

$$
\Psi:(t, y) \in(0, T) \times \Omega \mapsto \Omega
$$

belongs to $H^{2}\left(0, T ; \mathcal{C}^{2}(\Omega)\right)$ and is such that, for all $t \in(0, T), \Psi(t, \cdot)$ is a $\mathcal{C}^{2}$-diffeomorphism from $\Omega$ on $\Omega$ and from $S$ on $S(t)$. For a function $u(t, \cdot): S(t) \mapsto \mathbb{R}$, we define

$$
U(t, y)=u(t, \Psi(t, y)), \forall t \in(0, T), \forall y \in S .
$$

Then, we define, for all $1 \leq p, q \leq+\infty$, for all $k \in \mathbb{N}$,

$$
L^{p}\left(0, T ; W^{k, q}(S(t))\right)=\left\{u / U \in L^{p}\left(0, T ; W^{k, q}(S)\right)\right\},
$$

and, for $l=1,2$,

$$
W^{l, p}\left(0, T ; W^{k, q}(S(t))\right)=\left\{u / U \in W^{l, p}\left(0, T ; W^{k, q}(S)\right)\right\} .
$$

In each space, we consider the associated norms

$$
\|u\|_{L^{p}\left(0, T ; W^{k, q}(S(t))\right)}=\|U\|_{L^{p}\left(0, T ; W^{k, q}(S)\right)},\|u\|_{W^{l, p}\left(0, T ; W^{k, q}(S(t))\right)}=\|U\|_{W^{l, p}\left(0, T ; W^{k, q}(S)\right)} .
$$

We give some useful properties satisfied by these spaces.

Proposition 1. We use the same notations and hypotheses as in Definition 1.

- A function u belongs to $L^{p}\left(0, T ; W^{k, q}(S(t))\right)$ if and only if, for a.e. $t \in(0, T)$,

$$
x \mapsto u(t, x) \text { belongs to } W^{k, q}(S(t)) \text { and } \int_{0}^{T}\|u(t)\|_{\left.W^{k, q} S(t)\right)}^{p}<\infty .
$$

Moreover, the norm $\left(\int_{0}^{T}\|\cdot\|_{W^{k, q}(S(t))}^{p}\right)^{1 / p}$ is equivalent to $\|\cdot\|_{L^{p}\left(0, T ; W^{k, q}(S(t))\right)}$ :

$$
C_{1}\|u\|_{L^{p}\left(0, T ; W^{k, q}(S(t))\right)} \leq\left(\int_{0}^{T}\|u(t)\|_{W^{k, q}(S(t))}^{p}\right)^{1 / p} \leq C_{2}\|u\|_{L^{p}\left(0, T ; W^{k, q}(S(t))\right)},
$$

where $C_{1}>0$ and $C_{2}>0$ depend on the norm of $\Psi$ and $\Psi^{-1}$ in $L^{\infty}\left(0, T ; \mathcal{C}^{2}(\Omega)\right)$.

- If $u$ belongs to $W^{1, p}\left(0, T ; W^{k, q}(S(t))\right) \cap L^{p}\left(0, T ; W^{k+1, q}(S(t))\right)$, $\partial_{t} u$ defined by

$$
\partial_{t} u(t, x)=\partial_{t} U\left(t, \Psi^{-1}(t, x)\right)-\partial_{t} \Psi\left(t, \Psi^{-1}(t, x)\right) \cdot \nabla u(t, x)
$$

belongs to $L^{p}\left(0, T ; W^{k, q}(S(t))\right)$.

\subsection{Compatibility conditions on the initial data}

With (1.7), we have already given compatibility conditions which have to be satisfied by our initial data. In particular, we want the velocity to be continuous on the interface at initial time. These compatibility conditions are necessary to obtain a first regularity result on the velocities of the fluid and the structure (the precise result is given below by Prop. 2). Our study will also require a second regularity result on the acceleration associated 
to the fluid and structure motions (this result is given by Prop. 3). To obtain this result, we will need an additional compatibility condition expressing that the acceleration is continuous on the interface and on the global boundary at initial time. This kind of compatibility conditions appears for general classes of problems (we refer to [20] for a general theory).

First, we have to define the acceleration of the fluid and of the structure at time $t=0$. They will be determined by the equations of the motion as explained in the following lemma. Since our control function $f$ will be null at initial time, the compatibility condition will not depend on $f$.

Lemma 1. Let $u_{0} \in H^{3}\left(\Omega_{F}(0)\right)^{2}, a_{0} \in \mathbb{R}^{2}, a_{1} \in \mathbb{R}^{2}$ and $r_{0} \in \mathbb{R}$ be given. We consider the following problem

$$
\left\{\begin{array}{l}
u_{1}+\left(u_{0} \cdot \nabla\right) u_{0}-\operatorname{div} \sigma\left(u_{0}, p_{0}\right)=0 \text { in } \Omega_{F}(0), \\
m a_{2}=\int_{\partial \Omega_{S}(0)} \sigma\left(u_{0}, p_{0}\right) n \\
J r_{1}=\int_{\partial \Omega_{S}(0)}\left(\sigma\left(u_{0}, p_{0}\right) n\right) \cdot\left(x-a_{0}\right)^{\perp}, \\
\operatorname{div} u_{1}=0 \text { in } \Omega_{F}(0) \\
u_{1} \cdot n=0 \text { on } \partial \Omega \\
u_{1} \cdot n=\left(a_{2}+r_{1}\left(x-a_{0}\right)^{\perp}-r_{0}^{2}\left(x-a_{0}\right)-\nabla u_{0}\left(a_{1}+r_{0}\left(x-a_{0}\right)^{\perp}\right)\right) \cdot n \text { on } \partial \Omega_{S}(0) .
\end{array}\right.
$$

Then this problem admits a solution $\left(u_{1}, p_{0}, a_{2}, r_{1}\right) \in H^{1}\left(\Omega_{F}(0)\right)^{2} \times H^{2}\left(\Omega_{F}(0)\right) \times \mathbb{R}^{2} \times \mathbb{R}$. Moreover, this solution is unique (up to a constant for $p_{0}$ ).

Proof of Lemma 1. We define the solution $\left(u_{1,0}, p_{0,0}\right) \in H^{1}\left(\Omega_{F}(0)\right)^{2} \times H^{2}\left(\Omega_{F}(0)\right)$ obtained by a Helmholtz decomposition

$$
\left\{\begin{array}{l}
u_{1,0}+\nabla p_{0,0}=-\left(u_{0} \cdot \nabla\right) u_{0}+\Delta u_{0} \text { in } \Omega_{F}(0) \\
\operatorname{div} u_{1,0}=0 \text { in } \Omega_{F}(0), \\
u_{1,0} \cdot n=0 \text { on } \partial \Omega \\
u_{1,0} \cdot n=\left(-r_{0}^{2}\left(x-a_{0}\right)-\nabla u_{0}\left(a_{1}+r_{0}\left(x-a_{0}\right)^{\perp}\right)\right) \cdot n \text { on } \partial \Omega_{S}(0) .
\end{array}\right.
$$

In the sequel of the proof, we will denote by $x^{1}$ and $x^{2}$ the coordinates of a vector $x \in \mathbb{R}^{2}$. We consider the following problems:

$$
\left\{\begin{array}{l}
u_{1,1}+\nabla p_{0,1}=0 \text { in } \Omega_{F}(0) \\
\operatorname{div} u_{1,1}=0 \text { in } \Omega_{F}(0) \\
u_{1,1} \cdot n=0 \text { on } \partial \Omega \\
u_{1,1} \cdot n=n^{1} \text { on } \partial \Omega_{S}(0)
\end{array} \quad, \quad\left\{\begin{array}{l}
u_{1,2}+\nabla p_{0,2}=0 \text { in } \Omega_{F}(0) \\
\operatorname{div} u_{1,2}=0 \text { in } \Omega_{F}(0) \\
u_{1,2} \cdot n=0 \text { on } \partial \Omega \\
u_{1,2} \cdot n=n^{2} \text { on } \partial \Omega_{S}(0)
\end{array}\right.\right.
$$

and

$$
\left\{\begin{array}{l}
u_{1,3}+\nabla p_{0,3}=0 \text { in } \Omega_{F}(0) \\
\operatorname{div} u_{1,3}=0 \text { in } \Omega_{F}(0) \\
u_{1,3} \cdot n=0 \text { on } \partial \Omega \\
u_{1,3} \cdot n=\left(x-a_{0}\right)^{\perp} \cdot n \text { on } \partial \Omega_{S}(0) .
\end{array}\right.
$$

These three problems admit solutions in $H^{1}\left(\Omega_{F}(0)\right)^{2} \times H^{2}\left(\Omega_{F}(0)\right)$. We are looking for $u_{1}, p_{0}, a_{2}$ and $r_{1}$ satisfying, up to a constant for $p_{0}$,

$$
u_{1}=u_{1,0}+a_{2}^{1} u_{1,1}+a_{2}^{2} u_{1,2}+r_{1} u_{1,3}, p_{0}=p_{0,0}+a_{2}^{1} p_{0,1}+a_{2}^{2} p_{0,2}+r_{1} p_{0,3} .
$$


Thus, the dependence of $u_{1}$ and $p_{0}$ with respect to $a_{2}^{1}, a_{2}^{2}$ and $r_{1}$ is affine. From this expression, we deduce the system which has to be satisfied by $a_{2}$ and $r_{1}$

$$
\left\{\begin{array}{l}
m a_{2}^{1}=-a_{2}^{1} \int_{\partial \Omega_{S}(0)} p_{0,1} n^{1}-a_{2}^{2} \int_{\partial \Omega_{S}(0)} p_{0,2} n^{1}-r_{1} \int_{\partial \Omega_{S}(0)} p_{0,3} n^{1}+F_{1}^{1} \\
m a_{2}^{2}=-a_{2}^{1} \int_{\partial \Omega_{S}(0)} p_{0,1} n^{2}-a_{2}^{2} \int_{\partial \Omega_{S}(0)} p_{0,2} n^{2}-r_{1} \int_{\partial \Omega_{S}(0)} p_{0,3} n^{2}+F_{1}^{2} \\
J r_{1}=-a_{2}^{1} \int_{\partial \Omega_{S}(0)} p_{0,1} n \cdot\left(x-a_{0}\right)^{\perp}-a_{2}^{2} \int_{\partial \Omega_{S}(0)} p_{0,2} n \cdot\left(x-a_{0}\right)^{\perp}-r_{1} \int_{\partial \Omega_{S}(0)} p_{0,3} n \cdot\left(x-a_{0}\right)^{\perp}+F_{2}
\end{array}\right.
$$

with

$$
F_{1}=2 \int_{\partial \Omega_{S}(0)} \epsilon\left(u_{0}\right) n-\int_{\partial \Omega_{S}(0)} p_{0,0} n, F_{2}=2 \int_{\partial \Omega_{S}(0)}\left(\epsilon\left(u_{0}\right) n\right) \cdot\left(x-a_{0}\right)^{\perp}-\int_{\partial \Omega_{S}(0)}\left(p_{0,0} n\right) \cdot\left(x-a_{0}\right)^{\perp} .
$$

By noticing that, for instance,

$$
\int_{\partial \Omega_{S}(0)} p_{0,1} n^{1}=\int_{\Omega_{F}(0)}\left|u_{1,1}\right|^{2}, \int_{\partial \Omega_{S}(0)} p_{0,2} n^{1}=\int_{\Omega_{F}(0)} u_{1,1} \cdot u_{1,2},
$$

we can easily prove that, since $m>0$ and $J>0$, the matrix associated to this system is symmetric and definite positive. Thus, our system admits a unique solution $a_{2}^{1}, a_{2}^{2}$ and $r_{1}$ and then we deduce $u_{1}$ and $p_{0}$ from (1.11). $\square$

This lemma allows to define the acceleration $u_{1}$ of the fluid at initial time and the acceleration of the center of mass $a_{2}$ and of the angle $r_{1}$ at initial time. It asserts the continuity of the normal trace of the acceleration. In order to get the continuity of the whole trace of the acceleration, we make the following assumption on $\left(u_{1}, a_{2}, r_{0}\right)$ :

$$
u_{1}=0 \text { on } \partial \Omega, u_{1}=a_{2}+r_{1}\left(x-a_{0}\right)^{\perp}-r_{0}^{2}\left(x-a_{0}\right)-\nabla u_{0}\left(a_{1}+r_{0}\left(x-a_{0}\right)^{\perp}\right) \text { on } \partial \Omega_{S}(0) .
$$

Indeed, if we consider the expression (1.5) and we derive it with respect to time, we obtain this expression at initial time. To derive this expression, we have to be careful since the domain $\partial \Omega_{S}(t)$ depends on time. Thus, we first have to express this equality on $\partial \Omega_{S}(0)$ thanks to the flow $X$ defined by (1.8). Condition (1.12) can be expressed in terms of initial data $u_{0}, a_{0}, a_{1}$ and $r_{0}$.

We make the following hypothesis for $u_{0}, a_{0}, a_{1}, \theta_{0}$ and $r_{0}$ :

$$
\begin{aligned}
& u_{0} \in H^{3}\left(\Omega_{F}(0)\right)^{2}, a_{0} \in \mathbb{R}^{2}, a_{1} \in \mathbb{R}^{2}, \theta_{0} \in \mathbb{R} \text { and } r_{0} \in \mathbb{R}, \\
& \operatorname{div} u_{0}=0 \text { in } \Omega_{F}(0), u_{0}=a_{1}+r_{0}\left(x-a_{0}\right)^{\perp} \text { on } \partial \Omega_{S}(0) \text { and } u_{0}=0 \text { on } \partial \Omega, \\
& \left(u_{1}, a_{2}, r_{1}\right) \text { defined by Lemma } 1 \text { satisfy }(1.12) .
\end{aligned}
$$

\subsection{Main result}

We introduce the notion of controllability:

Definition 2. We will say that our problem is null controllable at time $T$ if there exists a control function $f \in L^{2}((0, T) \times \omega)^{2}$ such that

$$
u(T, \cdot)=0 \text { in } \Omega_{F}(T), a(T)=0, \dot{a}(T)=0, \theta(T)=0, r(T)=0
$$

or, equivalently,

$$
u(T, \cdot)=0 \text { in } \Omega, a(T)=0, \theta(T)=0,
$$

where $(u, a, \theta)$ is the solution, together with a pressure $p$, of the problem defined by equations (1.1) to (1.6). 
Thus, we want to drive the fluid and the structure at rest and we also want the structure to be located in the reference configuration $R_{-\theta_{0}}\left(\Omega_{S}(0)-a_{0}\right)$. The main result of this article is:

Theorem 1. We suppose that $u_{0}, a_{0}, a_{1}, \theta_{0}$ and $r_{0}$ satisfy (1.13) and we consider an initial structure domain $\Omega_{S}(0)$ such that (1.9) is satisfied. Let $T>0$ be a fixed final time. Then, there exists $\varepsilon>0$ depending on $T$ and on the domains $\Omega, \omega$ and $\Omega_{S}(0)$ such that, if

$$
\left\|u_{0}\right\|_{H^{3}\left(\Omega_{F}(0)\right)^{2}}+\left|a_{0}\right|+\left|a_{1}\right|+\left|\theta_{0}\right|+\left|r_{0}\right| \leq \varepsilon,
$$

the problem defined by equations (1.1) to (1.6) is null controllable at time T.

Remark 2. We can also consider $N$ structures occupying the domains $\Omega_{S}^{i}(t), 1 \leq i \leq N$, immersed in the fluid. The two equations for the structure motion are replaced by $2 N$ equations for the translation $a_{i}$ and the rotation velocity $r_{i}$ associated to the $i$-th solid. Each structure has to satisfy (1.9) and we also have to avoid contact between two different structures i.e.

$$
\mathrm{d}\left(\overline{\Omega_{S}^{i}(0)}, \overline{\Omega_{S}^{j}(0)}\right)>0, \forall 1 \leq i, j \leq N .
$$

Then we can prove that the same Carleman inequality (1.30) holds for the structure domain $\Omega_{S}(t)$ defined by $\Omega_{S}(t)=\bigcup_{1 \leq i \leq N} \Omega_{S}^{i}(t)$ and we can obtain the same local null controllability result.

Remark 3. By standard arguments in controllability, we can prove that this result also holds for a control domain located on the boundary of the cavity $\Omega$.

To begin with, we will prove a controllability result on a linearized problem. Let $(\tilde{a}, \tilde{r})$ be given in $H^{2}(0, T)^{2} \times$ $H^{1}(0, T)$. We define $\tilde{\theta}$ the angle associated to the rotation velocity $\tilde{r}$ defined up to a constant. Thus, for any $t \in(0, T)$, the structure domain $\widetilde{\Omega}_{S}(t)$ is defined by

$$
\widetilde{\Omega}_{S}(t)=\widetilde{X}\left(t, \Omega_{S}(0)\right),
$$

where $\widetilde{X}$ denotes the flow associated to the structure velocity and is defined by

$$
\widetilde{X}(t, y)=\tilde{a}(t)+R_{\tilde{\theta}(t)-\theta_{0}}\left(y-a_{0}\right), \forall t \in(0, T), \forall y \in \Omega_{S}(0) .
$$

We assume that $\tilde{a}$ and $\tilde{\theta}$ satisfy

$$
\tilde{a}(0)=a_{0}, \dot{\tilde{a}}(0)=a_{1}, \tilde{\theta}(0)=\theta_{0}, \tilde{r}(0)=r_{0}, \widetilde{\Omega}_{S}(t) \subset \Omega \backslash \omega, \mathrm{d}\left(\overline{\widetilde{\Omega}_{S}(t)}, \partial(\Omega \backslash \omega)\right) \geq \alpha, \forall t \in[0, T]
$$

where $\alpha>0$ is a fixed real number small enough. The last two properties are satisfied at time $t=0$ because $\widetilde{X}(0, \cdot)=\operatorname{Id}$ in $\Omega_{S}(0)$ and we have supposed that $\Omega_{S}(0)$ satisfies (1.9). We can also define the corresponding fluid domain by

$$
\widetilde{\Omega}_{F}(t)=\Omega \backslash \widetilde{\widetilde{\Omega}_{S}(t)}
$$

Next, let $\tilde{u}$ be given such that

$$
\begin{gathered}
\tilde{u} \in L^{\infty}\left(0, T ; L^{\infty}\left(\widetilde{\Omega}_{F}(t)\right)\right)^{2} \cap W^{1,4}\left(0, T ; L^{4}\left(\widetilde{\Omega}_{F}(t)\right)\right)^{2} \cap L^{\infty}\left(0, T ; H^{1}\left(\widetilde{\Omega}_{F}(t)\right)\right)^{2}, \\
\operatorname{div} \tilde{u}=0 \text { in } \widetilde{\Omega}_{F}(t), \tilde{u}=\dot{\tilde{a}}+\tilde{r}(x-\tilde{a})^{\perp} \text { on } \partial \widetilde{\Omega}_{S}(t), \tilde{u}=0 \text { on } \partial \Omega, \\
\tilde{u}(t=0)=u_{0} \text { in } \Omega_{F}(0) .
\end{gathered}
$$


As for the velocity $u$, we can extend $\tilde{u}$ on $\widetilde{\Omega}_{S}(t)$ by the velocity of the structure.

We will say that $(u, p, a, r)$ is a solution of the linearized problem around $(\tilde{u}, \tilde{a}, \tilde{r})$ if and only if, for all $t \in(0, T)$,

$$
\left\{\begin{array}{l}
\left(\partial_{t} u+(\tilde{u} \cdot \nabla) u\right)(t, x)-\operatorname{div} \sigma(u, p)(t, x)=f(t, x) 1_{\omega}(x), \forall x \in \widetilde{\Omega}_{F}(t), \\
m \ddot{a}(t)=\int_{\partial \tilde{\Omega}_{S}(t)} \sigma(u, p) n, \\
J \dot{r}(t)=\int_{\partial \tilde{\Omega}_{S}(t)}(\sigma(u, p) n) \cdot(x-\tilde{a}(t))^{\perp}, \\
\operatorname{div} u(t, x)=0, \forall x \in \widetilde{\Omega}_{F}(t), \\
u(t, x)=0, \forall x \in \partial \Omega, \\
u(t, x)=\dot{a}(t)+r(t)(x-\tilde{a}(t))^{\perp}, \forall x \in \partial \widetilde{\Omega}_{S}(t), \\
u(0, \cdot)=u_{0} \text { in } \Omega_{F}(0), a(0)=a_{0}, \dot{a}(0)=a_{1}, \theta(0)=\theta_{0}, r(0)=r_{0} .
\end{array}\right.
$$

We easily obtain an a priori energy estimate for this problem. Indeed denoting $\widetilde{E}(t)$ the global energy:

$$
\widetilde{E}(t)=\frac{1}{2} \int_{\widetilde{\Omega}_{F}(t)}|u(t, x)|^{2} \mathrm{~d} x+\frac{m}{2}|\dot{a}(t)|^{2}+\frac{J}{2}|r(t)|^{2}+\int_{0}^{t} \int_{\widetilde{\Omega}_{F}\left(t^{\prime}\right)}\left|\nabla u\left(t^{\prime}, x\right)\right|^{2} \mathrm{~d} x \mathrm{~d} t^{\prime},
$$

we have

$$
\widetilde{E}(t) \leq E(0)+C(T) \int_{0}^{t} \int_{\omega}\left|f\left(t^{\prime}, x\right)\right|^{2} \mathrm{~d} x \mathrm{~d} t^{\prime} .
$$

It seems worth noting that, in order to have an energy estimate for the linearized problem, the given velocities $\tilde{u}$, $\dot{\tilde{a}}$ and $\tilde{r}$ have to satisfy continuity and divergence-free conditions (1.19). Since the trace of $\tilde{u}$ has to be defined, we have taken $\tilde{u}$ in $L^{\infty}\left(0, T ; H^{1}\left(\widetilde{\Omega}_{F}(t)\right)\right)^{2}$.

First of all, we will prove a controllability result for this linearized problem. The result is formulated as follows:

Theorem 2. We consider initial data $u_{0} \in H^{1}\left(\Omega_{F}(0)\right)^{2}, a_{0} \in \mathbb{R}^{2}, a_{1} \in \mathbb{R}^{2}, \theta_{0} \in \mathbb{R}$ and $r_{0} \in \mathbb{R}$ satisfying (1.7) and an initial structure domain $\Omega_{S}(0)$ such that $(1.9)$ is satisfied.

Let $T>0$ be a fixed final time. We suppose that $(\tilde{a}, \tilde{r}) \in H^{2}(0, T)^{2} \times H^{1}(0, T)$ are such that (1.17) holds for some $\alpha>0$ and that $\tilde{u}$ satisfies conditions (1.18) to (1.20). Then, problem (1.21) is null controllable at time T.

To prove the controllability result for the linearized problem, we need to introduce the homogeneous adjoint problem. It is defined by the following system, for all $t \in(0, T)$

$$
\left\{\begin{array}{l}
\left(-\partial_{t} v-(\tilde{u} \cdot \nabla) v\right)(t, x)-\operatorname{div} \sigma(v, q)(t, x)=0, \forall x \in \widetilde{\Omega}_{F}(t), \\
m \ddot{b}(t)=-\int_{\partial \widetilde{\Omega}_{S}(t)} \sigma(v, q) n \\
J \dot{\gamma}(t)=-\int_{\partial \widetilde{\Omega}_{S}(t)}(\sigma(v, q) n) \cdot(x-\tilde{a}(t))^{\perp} \\
\operatorname{div} v(t, x)=0, \forall x \in \widetilde{\Omega}_{F}(t) \\
v(t, x)=0, \forall x \in \partial \Omega \\
v(t, x)=\dot{b}(t)+\gamma(t)(x-\tilde{a}(t))^{\perp}, \forall x \in \partial \widetilde{\Omega}_{S}(t), \\
v(T, \cdot)=v_{0}^{T} \text { in } \widetilde{\Omega}_{F}(T), b(T)=0, \dot{b}(T)=b_{1}^{T}, \gamma(T)=\gamma_{0}^{T}
\end{array}\right.
$$


The initial data $v_{0}^{T} \in H^{1}\left(\widetilde{\Omega}_{F}(T)\right)^{2}, b_{1}^{T}$ and $\gamma_{0}^{T}$ satisfy

$$
v_{0}^{T}=b_{1}^{T}+\gamma_{0}^{T}(x-\tilde{a}(T))^{\perp} \text { on } \partial \widetilde{\Omega}_{S}(T), v_{0}^{T}=0 \text { on } \partial \Omega \text { and } \operatorname{div} v_{0}^{T}=0 \text { in } \widetilde{\Omega}_{F}(T) .
$$

\subsection{Extension of the structure flow}

We have already introduced the definition of the structure flow by (1.16). In the following, we will need to extend this flow up to the global boundary $\partial \Omega$ by a regular and incompressible flow. To construct this extension, conditions of non-collision between the structure and the boundary of $\Omega$ have to be satisfied. According to condition (1.17), we have $\widetilde{\Omega}_{S}(t) \subset(\Omega \backslash \omega)_{\alpha}$, for each $t \in[0, T]$ where we have denoted, for a subset $A$ of $\mathbb{R}^{2}$, $A_{\epsilon}=\{x \in A / \mathrm{d}(x, \partial A) \geq \epsilon\}$. We have the following result:

Lemma 2. Let $(\tilde{a}, \tilde{r}) \in H^{2}(0, T)^{2} \times H^{1}(0, T)$ be given. We define $\widetilde{\Omega}_{S}(t)$ by (1.15) and we suppose that (1.17) is satisfied for some $\alpha>0$. We can extend the velocity

$$
\dot{\tilde{a}}+\tilde{r}(x-\tilde{a})^{\perp}
$$

defined on $\widetilde{\Omega}_{S}(t)$ by a velocity $\tilde{u}_{S} \in H^{1}\left(0, T ; \mathcal{C}^{2}(\Omega)\right)^{2}$ satisfying, for all $t \in(0, T)$

$$
\begin{aligned}
& \operatorname{div} \tilde{u}_{S}=0 \text { in } \Omega, \\
& \tilde{u}_{S}=0 \text { in } \Omega \backslash(\Omega \backslash \omega)_{\alpha / 4}, \tilde{u}_{S}=\dot{\tilde{a}}+\tilde{r}(x-\tilde{a})^{\perp} \text { in }(\Omega \backslash \omega)_{\alpha / 2},
\end{aligned}
$$

and such that

$$
\left\|\tilde{u}_{S}\right\|_{H^{1}\left(0, T ; \mathcal{C}^{2}(\Omega)\right)^{2}} \leq C\left(\|\dot{\tilde{a}}\|_{H^{1}(0, T)^{2}}+\|\tilde{r}\|_{H^{1}(0, T)}\right)
$$

where $C$ depends on $T$ and $\alpha$.

We do not detail how we obtain this incompressible velocity which extends the velocity defined on the structure: we refer to [19] for the proof of this result. We define the flow associated to $\tilde{u}_{S}$. We still denote it $\widetilde{X}$ since it extends the flow defined on the structure by (1.16).

Lemma 3. Under the same hypotheses as in Lemma 2 , the flow $\widetilde{X}$ associated to $\tilde{u}_{S}$ defined in Lemma 2 satisfies:

- for each $t \in[0, T], \widetilde{X}(t, \cdot)$ is a $\mathcal{C}^{2}$-diffeomorphism from $\Omega$ on $\Omega$ and from $\Omega_{F}(0)$ on $\widetilde{\Omega}_{F}(t)$. We denote by $\widetilde{Y}(t, \cdot)$ the inverse of $\widetilde{X}(t, \cdot)$ defined on $\Omega$;

- $\widetilde{X}$ and $\widetilde{Y}$ belong to $H^{2}\left(0, T ; \mathcal{C}^{2}(\Omega)\right)^{2}$;

- $\forall(t, y) \in(0, T) \times \Omega, \operatorname{det} \nabla \tilde{X}(t, y)=1$;

- $\forall t \in(0, T), \forall y \in \Omega \backslash(\Omega \backslash \omega)_{\alpha / 4}, \tilde{X}(t, y)=y$;

- $\forall t \in(0, T), \forall y \in \Omega_{S}(0)+B(0, \alpha / 2), \widetilde{X}(t, y)=\tilde{a}(t)+R_{\tilde{\theta}(t)-\theta_{0}}\left(y-a_{0}\right)$,

where $B(0, \alpha / 2)$ denotes the ball of center 0 and of radius $\alpha / 2$. Moreover, we have

$$
\|\tilde{X}\|_{H^{2}\left(0, T ; \mathcal{C}^{2}(\Omega)\right)^{2}}+\|\tilde{Y}\|_{H^{2}\left(0, T ; \mathcal{C}^{2}(\Omega)\right)^{2}} \leq C\left(\|\tilde{a}\|_{H^{2}(0, T)^{2}}+\|\tilde{r}\|_{H^{1}(0, T)}\right),
$$

where the constant $C$ depends on $T$ and $\alpha$.

Proof of Lemma 3 . Thanks to the regularity of $\tilde{u}_{S}$ obtained in Lemma 2 and the properties of the flow associated to a velocity, we easily obtain the first three points of the lemma.

Now, on $\Omega \backslash(\Omega \backslash \omega)_{\alpha / 4}$, since $\tilde{u}_{S}=0$, we have that $\widetilde{X}(t, \cdot)=$ Id. Moreover, for each $t \in(0, T)$, for each $y \in \Omega_{S}(0)+B(0, \alpha / 2)$, we have

$$
\tilde{a}(t)+R_{\tilde{\theta}(t)-\theta_{0}}\left(y-a_{0}\right) \in \widetilde{\Omega}_{S}(t)+B(0, \alpha / 2) \subset(\Omega \backslash \omega)_{\alpha / 2} .
$$

Consequently, by uniqueness of the flow, the last point of the lemma is satisfied. 
Remark 4. If $\tilde{a}$ belongs to $W^{1, \infty}(0, T)^{2}$ and $\tilde{r}$ belongs to $L^{\infty}(0, T)$, Lemmas 2 and 3 still hold with the appropriate changes (the flows belong to $W^{1, \infty}\left(0, T ; \mathcal{C}^{2}(\Omega)\right)^{2}$.

\subsection{A Carleman inequality}

To obtain our controllability result, we prove a Carleman inequality result for the adjoint system (1.22). We consider a nonempty open set $\omega_{0}$ such that $\omega_{0} \subset \subset \omega$ (i.e. $\overline{\omega_{0}} \subset \omega$ ). We will introduce time-dependent weight functions defined on the moving domain $\widetilde{\Omega}_{F}(t)$. First of all, we consider a steady weight function $\beta_{0}$ in $\mathcal{C}^{2}\left(\overline{\Omega_{F}(0)}\right)$ depending on $\Omega, \omega_{0}$ and $\Omega_{S}(0)$ such that

$$
\begin{aligned}
& \beta_{0}=0 \text { on } \partial \Omega \cup \partial \Omega_{S}(0), \beta_{0}>0 \text { in } \Omega_{F}(0), \\
& \nabla \beta_{0} \cdot n \leq c_{1}<0 \text { on } \partial \Omega, \nabla \beta_{0} \cdot n \geq c_{2}>0 \text { on } \partial \Omega_{S}(0),\left|\nabla \beta_{0}\right|>0 \text { in } \Omega_{F}(0) \backslash \overline{\omega_{0}} .
\end{aligned}
$$

On the boundary of $\Omega$, the vector $n$ is the outward unit normal to $\Omega$ and on the boundary of the structure domain, $n$ is the outward unit normal to the structure domain (and thus the inward normal to the fluid domain). For the proof of this result, we refer to [11]. We suppose that (1.17) holds for some $\alpha>0$. Then, thanks to $\beta_{0}$, we define the time-dependent weight function $\beta$ which follows the displacement of the structure by

$$
\beta(t, x)=\beta_{0}(\tilde{Y}(t, x)), \forall x \in \widetilde{\Omega}_{F}(t), \forall t \in(0, T),
$$

where $\tilde{Y}$ is defined by Lemma 3 .

Lemma 4. The function $\beta$ belongs to $L^{\infty}\left(0, T ; W^{2, \infty}\left(\widetilde{\Omega}_{F}(t)\right)\right) \cap W^{1, \infty}\left(0, T ; W^{1, \infty}\left(\widetilde{\Omega}_{F}(t)\right)\right)$ and is such that:

$$
\begin{aligned}
& \beta=0 \text { on } \partial \Omega \cup \partial \widetilde{\Omega}_{S}(t), \beta>0 \text { in } \widetilde{\Omega}_{F}(t) \\
& \nabla \beta \cdot n \leq c_{1}<0 \text { on } \partial \Omega, \nabla \beta \cdot n \geq c_{2}>0 \text { on } \partial \widetilde{\Omega}_{S}(t),|\nabla \beta|>0 \text { in } \widetilde{\Omega}_{F}(t) \backslash \overline{\omega_{0}}
\end{aligned}
$$

Moreover, we have the following estimate:

$$
\|\beta\|_{L^{\infty}\left(0, T ; W^{2, \infty}\left(\widetilde{\Omega}_{F}(t)\right)\right)}+\|\beta\|_{W^{1, \infty}\left(0, T ; W^{1, \infty}\left(\widetilde{\Omega}_{F}(t)\right)\right)} \leq C,
$$

where $C$ depends on $T$ and $\alpha$.

To introduce the Carleman inequality satisfied by a solution of the adjoint linearized problem (1.22), we define, for $\lambda \geq 1$, the functions $\mathcal{V}$ and $\varphi$ by: $\forall t \in(0, T), \forall x \in \widetilde{\Omega}_{F}(t)$,

$$
\mathcal{V}(t, x)=\frac{\mathrm{e}^{10 \lambda M}-\mathrm{e}^{\lambda(8 M+\beta(t, x))}}{t^{4}(T-t)^{4}}, \quad \varphi(t, x)=\frac{\mathrm{e}^{\lambda(8 M+\beta(t, x))}}{t^{4}(T-t)^{4}},
$$

where $M=\left\|\beta_{0}\right\|_{L^{\infty}\left(\Omega_{F}(0)\right)}$. For this choice of $M$, we can already notice that $\mathcal{V}$ is a positive function since $\|\beta\|_{L^{\infty}\left(0, T ; L^{\infty}\left(\widetilde{\Omega}_{F}(t)\right)\right)}=\left\|\beta_{0}\right\|_{L^{\infty}\left(\Omega_{F}(0)\right)}$. Moreover, $\mathcal{V}$ and $\varphi$ have the following properties:

$$
\nabla \mathcal{V}=-\lambda \varphi \nabla \beta, \quad \nabla \varphi=\lambda \varphi \nabla \beta
$$

We also define, $\forall t \in(0, T)$,

$$
\begin{aligned}
& \hat{\mathcal{V}}(t)=\inf _{x \in \widetilde{\Omega}_{F}(t)} \mathcal{V}(t, x)=\frac{\mathrm{e}^{10 \lambda M}-\mathrm{e}^{9 \lambda M}}{t^{4}(T-t)^{4}}, \mathcal{V}^{*}(t)=\sup _{x \in \widetilde{\Omega}_{F}(t)} \mathcal{V}(t, x)=\frac{\mathrm{e}^{10 \lambda M}-\mathrm{e}^{8 \lambda M}}{t^{4}(T-t)^{4}}, \\
& \hat{\varphi}(t)=\sup _{x \in \widetilde{\Omega}_{F}(t)} \varphi(t, x)=\frac{\mathrm{e}^{9 \lambda M}}{t^{4}(T-t)^{4}}, \varphi^{*}(t)=\inf _{x \in \widetilde{\Omega}_{F}(t)} \varphi(t, x)=\frac{\mathrm{e}^{8 \lambda M}}{t^{4}(T-t)^{4}} .
\end{aligned}
$$


Then, the following global Carleman estimate for problem (1.22) holds:

Theorem 3. Let $\tilde{a} \in H^{2}(0, T)^{2}, \tilde{r} \in H^{1}(0, T)$ and $\tilde{u}$ be given such that (1.17) holds for some $\alpha>0$ and such that conditions (1.18) to (1.20) are satisfied.

Then, there exists a constant $C$ and two constants $\hat{s}$ and $\hat{\lambda}$ such that, for every $v_{0}^{T} \in L^{2}\left(\widetilde{\Omega}_{F}(T)\right)^{2}, b_{1}^{T} \in \mathbb{R}^{2}$, $s_{0}^{T} \in \mathbb{R}$, the corresponding solution $(v, q, b, \gamma)$ of (1.22) satisfies, for any $s \geq \hat{s}$ and $\lambda \geq \hat{\lambda}$,

$$
\begin{gathered}
\int_{0}^{T} \int_{\widetilde{\Omega}_{F}(t)} \mathrm{e}^{-2 s \mathcal{V}}\left(\frac{1}{s \varphi}\left(|\Delta v|^{2}+\left|\partial_{t} v\right|^{2}\right)+s \lambda^{2} \varphi|\nabla v|^{2}+s^{3} \lambda^{4} \varphi^{3}|v|^{2}\right)+s \lambda \int_{0}^{T} \mathrm{e}^{-2 s \mathcal{V}^{*}} \varphi^{*}\left(|\ddot{b}|^{2}+|\dot{\gamma}|^{2}\right) \\
+s^{3} \lambda^{3} \int_{0}^{T} \int_{\partial \widetilde{\Omega}_{S}(t)} \mathrm{e}^{-2 s \mathcal{V}^{*}}\left(\varphi^{*}\right)^{3}|v|^{2}+s \lambda \int_{0}^{T} \int_{\partial \widetilde{\Omega}_{S}(t)} \mathrm{e}^{-2 s \mathcal{V}^{*}} \varphi^{*}|\nabla v n|^{2} \\
\leq C s^{19 / 2} \lambda^{13} \int_{0}^{T} \int_{\omega} \mathrm{e}^{2 s \mathcal{V}^{*}-4 s \hat{\mathcal{V}}} \hat{\varphi}^{10}|v|^{2}
\end{gathered}
$$

The constant $C$ only depends on $T, \alpha$ and $\beta_{0}$, and $\hat{s}$ and $\hat{\lambda}$ depend on $T, \alpha, \beta_{0}$ and the norm of $\tilde{a}$ in $H^{2}(0, T)^{2}$, $\tilde{r}$ in $H^{1}(0, T)$ and $\tilde{u}$ in $L^{\infty}((0, T) \times \Omega)^{2} \cap W^{1,4}\left(0, T ; L^{4}\left(\widetilde{\Omega}_{F}(t)\right)\right)^{2}$.

Remark 5. In this work, we suppose that the viscosity $\mu$ is equal to 1 . It can be interesting to wonder how the constant in our Carleman inequality depends on $\mu$ if $\mu$ is not fixed. It is known that the constant in global Carleman inequalities for parabolic equations behaves as $\exp (C / T)$, where $C>0$ is a constant depending on the domain and $T>0$ is the length of the time interval (see for instance [9]). Let us consider the heat equation

$$
u_{t}-\mu \Delta u=f \text { in }(0, T),
$$

where $\mu>0$ and make the change of variables $\tau=\mu t$ then we retrieve a heat equation with $\mu=1$

$$
\widetilde{u}_{\tau}-\Delta \widetilde{u}=\tilde{f} \text { in }(0, \mu T),
$$

where $\widetilde{u}(\tau)=u(\tau / \mu), \widetilde{f}(\tau)=f(\tau / \mu) / \mu$, and therefore, with the classical computations, we find that the constant in the global Carleman inequality is of order $\exp (C /(\mu T))$. It has been also shown that this constant is optimal for the observability inequality at least in one dimension (see [4]). In our case, the situation is essentially the same.

The proof of this theorem will be given in Section 2, but before, we will study some regularity properties which will be useful in the sequel.

\subsection{Regularity results on the linearized problem}

We give regularity results for the following non-homogeneous linearized system associated to (1.21): for all $t \in(0, T)$,

$$
\left\{\begin{array}{l}
\left(\partial_{t} u+(\tilde{u} \cdot \nabla) u\right)(t, x)-\operatorname{div} \sigma(u, p)(t, x)=g_{F}(t, x), \forall x \in \widetilde{\Omega}_{F}(t), \\
m \ddot{a}(t)=\int_{\partial \widetilde{\Omega}_{S}(t)} \sigma(u, p) n+g_{T}(t), \\
J \dot{r}(t)=\int_{\partial \widetilde{\Omega}_{S}(t)}(\sigma(u, p) n) \cdot(x-\tilde{a}(t))^{\perp}+g_{R}(t), \\
\operatorname{div} u(t, x)=0, \forall x \in \widetilde{\Omega}_{F}(t), \\
u(t, x)=0, \forall x \in \partial \Omega, \\
u(t, x)=\dot{a}(t)+r(t)(x-\tilde{a}(t))^{\perp}, \forall x \in \partial \widetilde{\Omega}_{S}(t), \\
u(0, \cdot)=u_{0} \text { in } \Omega_{F}(0), a(0)=a_{0}, \dot{a}(0)=a_{1}, \theta(0)=\theta_{0}, r(0)=r_{0},
\end{array}\right.
$$


where $g_{F}$ is the force acting on the fluid and $g_{T}$ and $g_{R}$ are the force and the torque acting on the structure. Of course, these results are also true for the linear adjoint system and can be shown in the same way. In [19], the first regularity result is proved for the nonlinear fluid-structure direct problem. Thus, the proposition which follows is a result contained in [19]. We only give a sketch of the proof and we refer to [19] and the references therein for complementary explanations. Let us define

$$
\mathcal{U}(0, T ; \Omega)=L^{2}\left(0, T ; H^{2}(\Omega)\right) \cap H^{1}\left(0, T ; L^{2}(\Omega)\right) \cap L^{\infty}\left(0, T ; H^{1}(\Omega)\right) .
$$

Proposition 2. Let initial data $u_{0} \in H^{1}\left(\Omega_{F}(0)\right)^{2}, a_{0} \in \mathbb{R}^{2}, a_{1} \in \mathbb{R}^{2}, \theta_{0} \in \mathbb{R}, r_{0} \in \mathbb{R}$ and forces $g_{F} \in$ $L^{2}\left(0, T ; L^{2}\left(\widetilde{\Omega}_{F}(t)\right)\right)^{2}, g_{T} \in L^{2}(0, T)^{2}, g_{R} \in L^{2}(0, T)$ be given. We suppose that initial data satisfy (1.7), that $\tilde{u} \in L^{\infty}\left(0, T ; L^{\infty}\left(\widetilde{\Omega}_{F}(t)\right)\right)^{2} \cap L^{\infty}\left(0, T ; H^{1}\left(\widetilde{\Omega}_{F}(t)\right)\right)^{2},(\tilde{a}, \tilde{r}) \in W^{1, \infty}(0, T)^{2} \times L^{\infty}(0, T)$ satisfy (1.19) and (1.17) for some $\alpha>0$. Then, the system (1.31) admits a unique solution

$$
u \in \mathcal{U}\left(0, T ; \widetilde{\Omega}_{F}(t)\right)^{2}, p \in L^{2}\left(0, T ; H^{1}\left(\widetilde{\Omega}_{F}(t)\right)\right), a \in H^{2}(0, T)^{2}, r \in H^{1}(0, T) .
$$

Moreover, we have the estimate

$$
\begin{aligned}
& \|u\|_{\mathcal{U}\left(0, T ; \tilde{\Omega}_{F}(t)\right)^{2}}+\|p\|_{L^{2}\left(0, T ; H^{1}\left(\tilde{\Omega}_{F}(t)\right)\right)}+\|a\|_{H^{2}(0, T)^{2}}+\|r\|_{H^{1}(0, T)} \\
& \leq C\left(\left\|\left(u_{0}, a_{1}, r_{0}\right)\right\|_{H^{1}\left(\Omega_{F}(0)\right)^{2} \times \mathbb{R}^{2} \times \mathbb{R}}+\left\|g_{F}\right\|_{L^{2}\left(0, T ; L^{2}\left(\widetilde{\Omega}_{F}(t)\right)\right)^{2}}+\left\|g_{T}\right\|_{L^{2}(0, T)^{2}}+\left\|g_{R}\right\|_{L^{2}(0, T)}\right),
\end{aligned}
$$

where the constant $C$ depends on $T, \alpha$, the norm of $\tilde{u}$ in $L^{\infty}((0, T) \times \Omega)^{2}$ (and thus on the norm of $(\tilde{a}, \tilde{r})$ in $\left.W^{1, \infty}(0, T)^{2} \times L^{\infty}(0, T)\right)$.

Proof of Proposition 2. This result is obtained by doing a change of variables to come back to initial configurations $\Omega_{F}(0)$ and $\Omega_{S}(0)$. Thanks to Lemma 3 (see Rem. 4), we define the flows $\widetilde{X}$ and $\widetilde{Y}$. Let us define the new variables

$$
U(t, y)=\nabla \tilde{Y}(t, \tilde{X}(t, y)) u(t, \widetilde{X}(t, y)), P(t, y)=p(t, \widetilde{X}(t, y)), A(t)=\int_{0}^{t} R_{\theta_{0}-\tilde{\theta}\left(t^{\prime}\right)} \dot{a}\left(t^{\prime}\right) \mathrm{d} t^{\prime} .
$$

It can be proved (see [19]) that $(u, p, a, r)$ is a solution of (1.31) if and only if $(U, P, A, r)$ satisfies

$$
\left\{\begin{array}{l}
\partial_{t} U-[L U]+[M U]+[N U, \widetilde{U}]+[G P]=G_{F} \text { in }(0, T) \times \Omega_{F}(0), \\
m \ddot{A}=\int_{\partial \Omega_{S}(0)} \sigma(U, P) n+G_{T}+m \tilde{r} \dot{A}^{\perp} \text { in }(0, T), \\
J \dot{r}=\int_{\partial \Omega_{S}(0)}(\sigma(U, P) n) \cdot\left(y-a_{0}\right)^{\perp}+G_{R} \text { in }(0, T), \\
\operatorname{div} U=0 \text { in }(0, T) \times \Omega_{F}(0), \\
U=0 \text { on }(0, T) \times \partial \Omega \\
U=\dot{A}+r\left(y-a_{0}\right)^{\perp} \text { on }(0, T) \times \partial \Omega_{S}(0), \\
U(0, \cdot)=u_{0} \text { in } \Omega_{F}(0), A(0)=0, \dot{A}(0)=a_{1}, r(0)=r_{0}
\end{array}\right.
$$

where we have defined

$$
\begin{aligned}
& G_{F}(t, y)=\nabla \tilde{Y}(t, \tilde{X}(t, y)) g_{F}(t, \tilde{X}(t, y)), G_{T}(t)=R_{\theta_{0}-\tilde{\theta}(t)} g_{T}(t), \\
& G_{R}(t)=g_{R}(t), \widetilde{U}(t, y)=\nabla \widetilde{Y}(t, \widetilde{X}(t, y)) \tilde{u}(t, \widetilde{X}(t, y)) .
\end{aligned}
$$


The operators $L, M, N$ and $G$ are given by (we implicitly sum over repeated indexes)

$$
\begin{aligned}
& {[L U]_{i}=\partial_{j}\left(g^{j k} \partial_{k} U_{i}\right)+2 g^{k l} \Gamma_{j k}^{i} \partial_{l} U_{j}+\left(\partial_{k}\left(g^{k l} \Gamma_{j l}^{i}\right)+g^{k l} \Gamma_{j l}^{m} \Gamma_{k m}^{i}\right) U_{j},} \\
& {[M U]_{i}=\left(\partial_{t} \widetilde{Y}_{j} \circ \widetilde{X}\right) \partial_{j} U_{i}+\left(\Gamma_{j k}^{i}\left(\partial_{t} \widetilde{Y}_{k} \circ \widetilde{X}\right)+\left(\partial_{k} \widetilde{Y}_{i} \circ \widetilde{X}\right) \partial_{t} \partial_{j} \widetilde{X}_{k}\right) U_{j}} \\
& {[N U, \widetilde{U}]_{i}=\widetilde{U}_{j} \partial_{j} U_{i}+\Gamma_{j k}^{i} \widetilde{U}_{j} U_{k}} \\
& {[G P]_{i}=g^{i j} \partial_{j} P}
\end{aligned}
$$

where we defined

$$
g^{i j}=\partial_{k} \tilde{Y}_{i} \circ \tilde{X} \partial_{k} \tilde{Y}_{j} \circ \tilde{X}, g_{i j}=\partial_{k} \widetilde{X}_{i} \partial_{k} \widetilde{X}_{j}, \Gamma_{i j}^{k}=\frac{1}{2} g^{k l}\left(\partial_{j} g_{i l}+\partial_{i} g_{j l}+\partial_{l} g_{i j}\right) .
$$

According to Lemma 5 which is given below and to definition 1 , we deduce the regularity result on the interval $\left(0, T_{0}\right)$. Since this time $T_{0}$ only depends on $\alpha$ and the norm of $\tilde{u}$ in $L^{\infty}((0, T) \times \Omega)^{2}$ and thanks to the estimate (1.34), we can extend our solution until time $T$.

Lemma 5. Let $G_{F} \in L^{2}\left(0, T ; L^{2}\left(\Omega_{F}(0)\right)\right)^{2}, G_{T} \in L^{2}(0, T)^{2}$ and $G_{R} \in L^{2}(0, T)$ be given. We consider initial data $u_{0} \in H^{1}\left(\Omega_{F}(0)\right)^{2}, a_{0} \in \mathbb{R}^{2}, a_{1} \in \mathbb{R}^{2}, \theta_{0} \in \mathbb{R}, r_{0} \in \mathbb{R}$ which satisfy (1.7) and we suppose that $\widetilde{U}$ belongs to $L^{\infty}\left((0, T) \times \Omega_{F}(0)\right)^{2}$, $\tilde{a}$ belongs to $W^{1, \infty}(0, T)^{2}$ and $\tilde{r}$ belongs to $L^{\infty}(0, T)$. We consider the system (1.32) where $L, M, N$ and $G$ are defined by (1.33).

Then there exists a time $0<T_{0}<T$ depending on $\alpha$, the norm of $\widetilde{U}$ in $L^{\infty}\left((0, T) \times \Omega_{F}(0)\right)^{2}, \tilde{a}$ in $W^{1, \infty}(0, T)^{2}$ and $\tilde{r}$ in $L^{\infty}(0, T)$ such that this system admits a unique solution

$$
U \in \mathcal{U}\left(0, T_{0} ; \Omega_{F}(0)\right)^{2}, P \in L^{2}\left(0, T_{0} ; H^{1}\left(\Omega_{F}(0)\right)\right), A \in H^{2}\left(0, T_{0}\right)^{2}, r \in H^{1}\left(0, T_{0}\right) .
$$

Moreover, we have the estimate

$$
\begin{aligned}
& \|U\|_{\mathcal{U}\left(0, T_{0} ; \Omega_{F}(0)\right)^{2}}+\|P\|_{L^{2}\left(0, T_{0} ; H^{1}\left(\Omega_{F}(0)\right)\right)}+\|A\|_{H^{2}\left(0, T_{0}\right)^{2}}+\|r\|_{H^{1}\left(0, T_{0}\right)} \\
& \leq C\left(\left\|\left(u_{0}, a_{1}, r_{0}\right)\right\|_{H^{1}\left(\Omega_{F}(0)\right)^{2} \times \mathbb{R}^{2} \times \mathbb{R}}+\left\|G_{F}\right\|_{L^{2}\left(0, T_{0} ; L^{2}\left(\Omega_{F}(0)\right)\right)^{2}}+\left\|G_{T}\right\|_{L^{2}\left(0, T_{0}\right)^{2}}+\left\|G_{R}\right\|_{L^{2}\left(0, T_{0}\right)}\right)
\end{aligned}
$$

where the constant $C$ depends on $T$, $\alpha$, the norms of $\widetilde{U}$ in $L^{\infty}\left((0, T) \times \Omega_{F}(0)\right)^{2}$, $\tilde{a}$ in $W^{1, \infty}(0, T)^{2}$ and $\tilde{r}$ in $L^{\infty}(0, T)$.

Proof of Lemma 5. First of all, we consider the linear problem

$$
\left\{\begin{array}{l}
\partial_{t} U-\Delta U+\nabla P=F_{F} \text { in }(0, T) \times \Omega_{F}(0), \\
m \ddot{A}=\int_{\partial \Omega_{S}(0)} \sigma(U, P) n+F_{T} \text { in }(0, T), \\
J \dot{r}=\int_{\partial \Omega_{S}(0)}(\sigma(U, P) n) \cdot\left(y-a_{0}\right)^{\perp}+F_{R} \text { in }(0, T), \\
\operatorname{div} U=0 \text { in }(0, T) \times \Omega_{F}(0), \\
U=0 \text { on }(0, T) \times \partial \Omega, \\
U=\dot{A}+r\left(y-a_{0}\right)^{\perp} \text { on }(0, T) \times \partial \Omega_{S}(0), \\
U(0, \cdot)=u_{0} \text { in } \Omega_{F}(0), A(0)=0, \dot{A}(0)=a_{1}, r(0)=r_{0} .
\end{array}\right.
$$

A regularity result for this problem is proved in [19]: this system admits a unique solution $(U, P, A, r)$ in $\mathcal{U}\left(0, T ; \Omega_{F}(0)\right)^{2} \times L^{2}\left(0, T ; H^{1}\left(\Omega_{F}(0)\right)\right) \times H^{2}(0, T)^{2} \times H^{1}(0, T)$ and (1.34) is satisfied with $G_{F}, G_{T}$ and $G_{R}$ 
respectively replaced by $F_{F}, F_{T}$ and $F_{R}$. $(U, P, A, r)$ is solution of the system (1.32) if and only if it is solution of (1.35) with

$$
\begin{aligned}
& F_{F}=G_{F}+[(L-\Delta) U]-[M U]-[N U, \widetilde{U}]+[(\nabla-G) P], \\
& F_{T}=G_{T}+m \tilde{r} \dot{A}^{\perp}, F_{R}=G_{R} .
\end{aligned}
$$

By proving estimates on the coefficients as in [19] thanks to Lemma 3 and Remark 4, we obtain that, near 0, the flows $\widetilde{X}$ and $\widetilde{Y}$ stay close to $I d$ and thus, the operators $L-\Delta, M, N, \nabla-G$ stay small. Therefore, we obtain the regularity result.

We will also need estimates on the second derivative of the velocity for the adjoint linear problem and the direct linear problem. The result which follows is given for the direct linear problem but it can be word for word adapted to the adjoint linear problem. This result plays a key role to prove Carleman estimate and to pass to the nonlinear problem in the last section. Hypotheses of regularity (1.18) we have to do come directly from this proposition. We suppose that the given forces satisfy

$$
g_{F}(t=0)=0 \text { in } \Omega_{F}(0), g_{T}(t=0)=0, g_{R}(t=0)=0 .
$$

Proposition 3. Let $g_{F} \in H^{1}\left(0, T ; L^{2}\left(\widetilde{\Omega}_{F}(t)\right)\right)^{2}, g_{T} \in H^{1}(0, T)^{2}, g_{R} \in H^{1}(0, T)$ be given functions satisfying (1.36). We suppose that initial data $u_{0}, a_{0}, a_{1}, \theta_{0}$ and $r_{0}$ satisfy $(1.13)$ and we consider $\tilde{u}$ and $(\tilde{a}, \tilde{r}) \in$ $H^{2}(0, T)^{2} \times H^{1}(0, T)$ satisfying conditions (1.17) to (1.20). Then, the system (1.31) admits a unique solution

$$
\begin{aligned}
& u \in H^{1}\left(0, T ; H^{2}\left(\widetilde{\Omega}_{F}(t)\right)\right)^{2} \cap H^{2}\left(0, T ; L^{2}\left(\widetilde{\Omega}_{F}(t)\right)\right)^{2} \cap W^{1, \infty}\left(0, T ; H^{1}\left(\widetilde{\Omega}_{F}(t)\right)\right)^{2}, \\
& p \in H^{1}\left(0, T ; H^{1}\left(\widetilde{\Omega}_{F}(t)\right)\right), a \in H^{3}(0, T)^{2}, r \in H^{2}(0, T) .
\end{aligned}
$$

Moreover, we have the estimate

$$
\begin{aligned}
& \|u\|_{H^{1}\left(0, T ; H^{2}\left(\widetilde{\Omega}_{F}(t)\right)\right)^{2}}+\|u\|_{H^{2}\left(0, T ; L^{2}\left(\widetilde{\Omega}_{F}(t)\right)\right)^{2}}+\|u\|_{W^{1, \infty}\left(0, T ; H^{1}\left(\widetilde{\Omega}_{F}(t)\right)\right)^{2}}+\|p\|_{H^{1}\left(0, T ; H^{1}\left(\widetilde{\Omega}_{F}(t)\right)\right)} \\
& +\|a\|_{H^{3}(0, T)^{2}}+\|r\|_{H^{2}(0, T)} \leq C\left(\left\|\left(u_{0}, a_{1}, r_{0}\right)\right\|_{H^{3}\left(\Omega_{F}(0)\right)^{2} \times \mathbb{R}^{2} \times \mathbb{R}}+\left\|g_{F}\right\|_{H^{1}\left(0, T ; L^{2}\left(\widetilde{\Omega}_{F}(t)\right)\right)^{2}}\right. \\
& \left.+\left\|g_{T}\right\|_{H^{1}(0, T)^{2}}+\left\|g_{R}\right\|_{H^{1}(0, T)}\right)
\end{aligned}
$$

where the constant $C$ depends on $T$, $\alpha$, the norm of $\tilde{u}$ in $L^{\infty}\left(0, T ; L^{\infty}\left(\widetilde{\Omega}_{F}(t)\right)\right)^{2}$ and in $W^{1,4}\left(0, T ; L^{4}\left(\widetilde{\Omega}_{F}(t)\right)\right)^{2}$ and the norm of $(\tilde{a}, \tilde{r})$ in $H^{2}(0, T)^{2} \times H^{1}(0, T)$.

Proof of Proposition 3. As in Proposition 2, we consider the equivalent transported system (1.32) on $\Omega_{F}(0)$. We derive this system with respect to time. We see that $\left(\partial_{t} U, \partial_{t} P, \dot{A}, \dot{r}\right)$ is formally solution of

$$
\left\{\begin{array}{l}
\partial_{t} W-[L W]+[M W]+[N W, \widetilde{U}]+[G \Pi]=\mathcal{G}_{F} \text { in }(0, T) \times \Omega_{F}(0), \\
m \ddot{D}=\int_{\partial \Omega_{S}(0)} \sigma(W, \Pi) n+\mathcal{G}_{T}-m \tilde{r} \dot{D}^{\perp} \text { in }(0, T), \\
J \dot{\tau}=\int_{\partial \Omega_{S}(0)}(\sigma(W, \Pi) n) \cdot\left(y-a_{0}\right)^{\perp}+\mathcal{G}_{R} \text { in }(0, T), \\
\operatorname{div} W=0 \text { in }(0, T) \times \Omega_{F}(0), \\
W=0 \text { on }(0, T) \times \partial \Omega \\
W=\dot{D}+\tau\left(y-a_{0}\right)^{\perp} \text { on }(0, T) \times \partial \Omega_{S}(0), \\
W(0, \cdot)=\partial_{t} U(0, \cdot) \text { in } \Omega_{F}(0), D(0)=\dot{A}(0), \dot{D}(0)=\ddot{A}(0), \tau(0)=\dot{r}(0),
\end{array}\right.
$$


where the forces are given by

$$
\begin{aligned}
\mathcal{G}_{F} & =\partial_{t} G_{F}+\left[\partial_{t} L U\right]-\left[\partial_{t} M U\right]-\left[\partial_{t} N U, \widetilde{U}\right]-\left[N U, \partial_{t} \widetilde{U}\right]-\left[\partial_{t} G P\right] \\
\mathcal{G}_{T} & =\partial_{t} G_{T}+m \dot{\tilde{r}} \dot{A}^{\perp} \\
\mathcal{G}_{R} & =\partial_{t} G_{R} .
\end{aligned}
$$

We have defined $\partial_{t} L, \partial_{t} M, \partial_{t} N$ and $\partial_{t} G$ by

$$
\begin{aligned}
& {\left[\partial_{t} L U\right]_{i}=\partial_{j}\left(\partial_{t} g^{j k} \partial_{k} U_{i}\right)+2 \partial_{t}\left(g^{k l} \Gamma_{j k}^{i}\right) \partial_{l} U_{j}+\left(\partial_{t} \partial_{k}\left(g^{k l} \Gamma_{j l}^{i}\right)+\partial_{t}\left(g^{k l} \Gamma_{j l}^{m} \Gamma_{k m}^{i}\right)\right) U_{j},} \\
& {\left[\partial_{t} M U\right]_{i}=\partial_{t}\left(\partial_{t} \widetilde{Y}_{j} \circ \widetilde{X}\right) \partial_{j} U_{i}+\partial_{t}\left(\Gamma_{j k}^{i}\left(\partial_{t} \widetilde{Y}_{k} \circ \widetilde{X}\right)+\left(\partial_{k} \widetilde{Y}_{i} \circ \widetilde{X}\right) \partial_{t} \partial_{j} \widetilde{X}_{k}\right) U_{j},} \\
& {\left[\partial_{t} N U, \widetilde{U}\right]_{i}=\partial_{t} \Gamma_{j k}^{i} \widetilde{U}_{j} U_{k},} \\
& {\left[\partial_{t} G P\right]_{i}=\partial_{t} g^{i j} \partial_{j} P .}
\end{aligned}
$$

These operators correspond to $L, M, N$ and $G$ where we have derived the coefficients with respect to time. Lemma 1 allows to define the initial data $\partial_{t} U(0, \cdot), \ddot{A}(0)$ and $\dot{r}(0)$.

We do not prove here rigorously that $\left(\partial_{t} U, \partial_{t} P, \dot{A}, \dot{r}\right)$ satisfies this system. This can be done by considering the solution $(W, \Pi, D, \tau)$ of the problem (1.38). According to what follows, this solution is well defined. Then, it can be proved that the primitives in time of this solution with the good initial data satisfy the same problem as $(U, P, A, r)$ and thus, by uniqueness, we can identify $(W, \Pi, D, \tau)$ and $\left(\partial_{t} U, \partial_{t} P, \dot{A}, \dot{r}\right)$.

We want to apply the regularity result given by Lemma 5 to system (1.38). First, according to Lemma 1 , $\partial_{t} U(0, \cdot)$ belongs to $H^{1}\left(\Omega_{F}(0)\right)^{2}$ and, since the acceleration of the fluid at initial time $u_{1}$ and the terms of acceleration of the structure at initial time $a_{2}$ and $r_{1}$ satisfy (1.12), the compatibility conditions

$$
\operatorname{div} \partial_{t} U(0, \cdot)=0 \text { in } \Omega_{F}(0), \partial_{t} U(0, y)=0 \text { on } \partial \Omega, \partial_{t} U(0, y)=\ddot{A}(0)+\dot{r}(0)\left(y-a_{0}\right) \text { on } \partial \Omega_{S}(0)
$$

are satisfied. Next, we have to prove estimates on $\mathcal{G}_{F}, \mathcal{G}_{T}$ and $\mathcal{G}_{R}$. According to Lemma 5, $(U, P, A, r)$ satisfies (1.34) on $\left(0, T_{0}\right)$ where $T_{0}$ depends on $\alpha$ and the norm of $\tilde{u}$ in $L^{\infty}((0, T) \times \Omega)^{2}$. Next, we notice that the coefficients $g_{i j}, g^{i j}$ and $\Gamma_{i j}^{k}$ belong to $H^{2}\left(0, T ; \mathcal{C}^{2}\left(\Omega_{F}(0)\right)\right)$ since $\widetilde{X}$ and $\widetilde{Y}$ belong to $H^{2}\left(0, T ; \mathcal{C}^{2}(\Omega)\right)$. Thus, we have

$$
\begin{aligned}
& \left\|\left[\partial_{t} L U\right]-\left[\partial_{t} M U\right]-\left[\partial_{t} N U, \widetilde{U}\right]\right\|_{L^{2}\left(\left(0, T_{0}\right) \times \Omega_{F}(0)\right)^{2}} \leq C\|U\|_{\mathcal{U}\left(0, T_{0} ; \Omega_{F}(0)\right)^{2}}, \\
& \left\|\left[\partial_{t} G P\right]\right\|_{L^{2}\left(\left(0, T_{0}\right) \times \Omega_{F}(0)\right)^{2}} \leq C\|P\|_{L^{2}\left(0, T_{0} ; H^{1}\left(\Omega_{F}(0)\right)\right)},
\end{aligned}
$$

where $C$ depends on $T$, $\alpha$, the norm of $\widetilde{U}$ in $L^{\infty}\left((0, T) \times \Omega_{F}(0)\right)^{2}$ and the norm of $(\tilde{a}, \tilde{r})$ in $H^{2}(0, T)^{2} \times H^{1}(0, T)$.

Moreover, since $U$ belongs to $\mathcal{U}\left(0, T_{0} ; \Omega_{F}(0)\right)^{2} \hookrightarrow L^{4-\alpha}\left(0, T_{0} ; W^{1,4-\alpha}\left(\Omega_{F}(0)\right)\right)^{2}$ for every $\alpha>0$ and $\partial_{t} \widetilde{U}$ belongs $L^{4}\left((0, T) \times \Omega_{F}(0)\right)^{2}$, we have

$$
\left\|\left[N U, \partial_{t} \widetilde{U}\right]\right\|_{L^{2}\left(\left(0, T_{0}\right) \times \Omega_{F}(0)\right)^{2}} \leq C\|U\|_{\mathcal{U}\left(0, T_{0} ; \Omega_{F}(0)\right)^{2}} .
$$

Thus, thanks to (1.34), we obtain an estimate on $\mathcal{G}_{F}$ in $L^{2}\left(0, T_{0} ; L^{2}\left(\Omega_{F}(0)\right)\right)^{2}$ :

$$
\begin{aligned}
\left\|\mathcal{G}_{F}\right\|_{L^{2}\left(\left(0, T_{0}\right) \times \Omega_{F}(0)\right)^{2}} \leq & C\left(\left\|\left(u_{0}, a_{1}, r_{0}\right)\right\|_{H^{1}\left(\Omega_{F}(0)\right)^{2} \times \mathbb{R}^{2} \times \mathbb{R}}+\left\|G_{F}\right\|_{H^{1}\left(0, T_{0} ; L^{2}\left(\Omega_{F}(0)\right)\right)^{2}}\right. \\
& \left.+\left\|G_{T}\right\|_{L^{2}\left(0, T_{0}\right)^{2}}+\left\|G_{R}\right\|_{L^{2}\left(0, T_{0}\right)}\right),
\end{aligned}
$$

where the constant $C$ depends on $\alpha$, the norm of $\tilde{u}$ in $L^{\infty}\left(0, T ; L^{\infty}\left(\widetilde{\Omega}_{F}(t)\right)\right)^{2}$ and in $W^{1,4}\left(0, T ; L^{4}\left(\widetilde{\Omega}_{F}(t)\right)\right)^{2}$ and on the norm of $(\tilde{a}, \tilde{r})$ in $H^{2}(0, T)^{2} \times H^{1}(0, T)$. 
For $\mathcal{G}_{T}$ and $\mathcal{G}_{R}$, we also have estimates in $L^{2}(0, T)$ :

$$
\begin{aligned}
\left\|\mathcal{G}_{T}\right\|_{L^{2}\left(0, T_{0}\right)^{2} \leq} \leq & C\left(\left\|\left(u_{0}, a_{1}, r_{0}\right)\right\|_{H^{1}\left(\Omega_{F}(0)\right)^{2} \times \mathbb{R}^{2} \times \mathbb{R}}+\left\|G_{F}\right\|_{L^{2}\left(0, T_{0} ; L^{2}\left(\Omega_{F}(0)\right)\right)^{2}}\right. \\
& \left.+\left\|G_{T}\right\|_{H^{1}\left(0, T_{0}\right)^{2}}+\left\|G_{R}\right\|_{L^{2}\left(0, T_{0}\right)}\right), \\
\left\|\mathcal{G}_{R}\right\|_{L^{2}\left(0, T_{0}\right)} \leq & \left\|G_{R}\right\|_{H^{1}\left(0, T_{0}\right)} .
\end{aligned}
$$

We deduce from Lemma 5 that

$$
\partial_{t} U \in \mathcal{U}\left(0, T_{0} ; \Omega_{F}(0)\right)^{2}, \partial_{t} P \in L^{2}\left(0, T_{0} ; H^{1}\left(\Omega_{F}(0)\right)\right), A \in H^{3}\left(0, T_{0}\right)^{2}, r \in H^{2}\left(0, T_{0}\right),
$$

and

$$
\begin{aligned}
& \left\|\partial_{t} U\right\|_{\mathcal{U}\left(0, T_{0} ; \Omega_{F}(0)\right)^{2}}+\left\|\partial_{t} P\right\|_{L^{2}\left(0, T_{0} ; H^{1}\left(\Omega_{F}(0)\right)\right)}+\|A\|_{H^{3}\left(0, T_{0}\right)^{2}}+\|r\|_{H^{2}\left(0, T_{0}\right)} \\
& \leq C\left(\left\|\left(u_{0}, a_{1}, r_{0}\right)\right\|_{H^{3}\left(\Omega_{F}(0)\right)^{2} \times \mathbb{R}^{2} \times \mathbb{R}}+\left\|G_{F}\right\|_{H^{1}\left(0, T_{0} ; L^{2}\left(\Omega_{F}(0)\right)\right)^{2}}+\left\|G_{T}\right\|_{H^{1}\left(0, T_{0}\right)^{2}}+\left\|G_{R}\right\|_{H^{1}\left(0, T_{0}\right)}\right) .
\end{aligned}
$$

Thus, we obtain our proposition and estimate (1.37) on $\left(0, T_{0}\right)$. Next, we can extend this solution until time $T$ since this time $T_{0}$ only depends on $\alpha$ and the norm of $\tilde{u}$ in $L^{\infty}((0, T) \times \Omega)^{2}$.

The first step of our work is devoted to the proof of the Carleman inequality.

\section{Proof of Theorem 3}

We divide the proof of Theorem 3 in several subsections: in the first subsection, the Navier-Stokes equation is treated as a heat equation with a right-hand side depending on the pressure. We have to be careful since the fluid domain (and consequently the associated weight functions) depends on time. Thanks to the equations satisfied by the structure, we obtain estimates on the acceleration of the rigid motion which enable us to bound the terms on the interface appearing in the Carleman estimate. We obtain an inequality with global integrals in the pressure in the right-hand side. In the second subsection, we prove that we can replace this global integral in the pressure by a local integral: to do this, we follow the method introduced by [10] using an auxiliary Carleman inequality given in [13]. And finally, in the last subsection, we estimate this local integral in the pressure, following the arguments in [10]. The hypotheses of Theorem 3 on the acceleration of the given motion $(\tilde{u}, \tilde{a}, \tilde{r})$ are only used in this last section.

\subsection{A first estimate with global integrals in the pressure in the right-hand side}

We set:

$$
w(t, x)=\mathrm{e}^{-s \mathcal{V}(t, x)} v(t, x), \forall x \in \widetilde{\Omega}_{F}(t), \forall t \in(0, T),
$$

where $v$ together with some $q, b$ and $\gamma$ is the solution of the homogeneous adjoint problem (1.22). Notice that $w$ satisfies

$$
\begin{aligned}
& w(0, x)=0, \forall x \in \Omega_{F}(0), w(T, x)=0, \forall x \in \widetilde{\Omega}_{F}(T), \\
& w(t, x)=0, \forall t \in(0, T), \forall x \in \partial \Omega, \\
& w(t, x)=\mathrm{e}^{-s \mathcal{V}^{*}(t)}\left(\dot{b}(t)+\gamma(t)(x-\tilde{a}(t))^{\perp}\right), \forall t \in(0, T), \forall x \in \partial \widetilde{\Omega}_{S}(t) .
\end{aligned}
$$


We have

$$
\begin{aligned}
& \partial_{t} v=\mathrm{e}^{s \mathcal{V}}\left(\partial_{t} w+s \partial_{t} \mathcal{V} w\right), \nabla v=\mathrm{e}^{s \mathcal{V}}\left(\nabla w+s w \nabla \mathcal{V}^{t}\right) \\
& \Delta v=\mathrm{e}^{s \mathcal{V}}\left(\Delta w+2 s \nabla w \nabla \mathcal{V}+s \Delta \mathcal{V} w+s^{2}|\nabla \mathcal{V}|^{2} w\right)
\end{aligned}
$$

Replacing $v$ by $w$ in the first equation of the adjoint problem (1.22), we obtain that

$$
-\partial_{t} w-s \partial_{t} \mathcal{V} w-(\tilde{u} \cdot \nabla) w-\Delta w-2 s \nabla w \nabla \mathcal{V}-s^{2}|\nabla \mathcal{V}|^{2} w-s \Delta \mathcal{V} w=s(\tilde{u} \cdot \nabla \mathcal{V}) w-\mathrm{e}^{-s \mathcal{V}} \nabla q,
$$

so equivalently

$$
L_{1}(w)+L_{2}(w)=g_{s}
$$

where $L_{1}(w), L_{2}(w)$ and $g_{s}$ are defined by

$$
\begin{aligned}
L_{1}(w) & =-\Delta w-s^{2} \lambda^{2} \varphi^{2}|\nabla \beta|^{2} w \\
L_{2}(w) & =-\left(\partial_{t} w+\left(\tilde{u}_{S} \cdot \nabla\right) w\right)+2 s \lambda \varphi \nabla w \nabla \beta+2 s \lambda^{2} \varphi|\nabla \beta|^{2} w, \\
g_{s} & =s \Delta \mathcal{V} w+2 s \lambda^{2} \varphi|\nabla \beta|^{2} w+s\left(\partial_{t} \mathcal{V}+\tilde{u} \cdot \nabla \mathcal{V}\right) w-\left(\left(\tilde{u}_{S}-\tilde{u}\right) \cdot \nabla\right) w-\mathrm{e}^{-s \mathcal{V}} \nabla q .
\end{aligned}
$$

Let us notice that we have added the term $2 s \lambda^{2} \varphi|\nabla \beta|^{2} w$ in $L_{2}$ and in $g_{s}$ in order to obtain additional estimates on $\nabla w$. We recall that the velocity $\tilde{u}_{S}$ is defined by Lemma 2 . Then, we have

$$
\left\|L_{1}(w)\right\|_{2}^{2}+\left\|L_{2}(w)\right\|_{2}^{2}+2\left(L_{1}(w), L_{2}(w)\right)_{2}=\left\|g_{s}\right\|_{2}^{2}
$$

where $(\cdot, \cdot)_{2}$ and $\|\cdot\|_{2}$ denote the following scalar product and the associated norm:

$$
(U, V)_{2}=\int_{0}^{T} \int_{\widetilde{\Omega}_{F}(t)} U(t, x) \cdot V(t, x), \quad\|U\|_{2}^{2}=(U, U)_{2} .
$$

We shall now compute the scalar product in the left hand side of (2.8). We can write that

$$
\left(L_{1}(w), L_{2}(w)\right)_{2}=\sum_{i=1}^{2} \sum_{j=1}^{3} I_{i j}
$$

where $I_{i j}$ represents the scalar product between the $i$-th term of $L_{1}(w)$ and the $j$-th term of $L_{2}(w)$. In the sequel, these two integrals will play a key role:

$$
K_{1}=s^{3} \lambda^{4} \int_{0}^{T} \int_{\widetilde{\Omega}_{F}(t)} \varphi^{3}|w|^{2}, \quad K_{2}=s \lambda^{2} \int_{0}^{T} \int_{\widetilde{\Omega}_{F}(t)} \varphi|\nabla w|^{2} \text {, for } s>1 \text { and } \lambda>1 .
$$

We have the following successive results:

$$
\begin{aligned}
I_{11} & =\int_{0}^{T} \int_{\widetilde{\Omega}_{F}(t)} \Delta w \cdot\left(\partial_{t} w+\left(\tilde{u}_{S} \cdot \nabla\right) w\right) \\
& =-\int_{0}^{T} \int_{\widetilde{\Omega}_{F}(t)} \nabla w: \nabla\left(\partial_{t} w+\left(\tilde{u}_{S} \cdot \nabla\right) w\right)-\int_{0}^{T} \int_{\partial \widetilde{\Omega}_{S}(t)}(\nabla w n) \cdot\left(\partial_{t} w+\left(\tilde{u}_{S} \cdot \nabla\right) w\right) .
\end{aligned}
$$

This is obtained by integrating by parts in space. We recall that $n$ denotes the outward unit normal to $\partial \widetilde{\Omega}_{S}(t)$. Since we are working on moving domains, we have to be careful on the way we treat the integral. We recall a 
differentiation formula: for a scalar function $f$ regular enough, we have

$$
\frac{\mathrm{d}}{\mathrm{d} t} \int_{\tilde{\Omega}_{F}(t)} f=\int_{\tilde{\Omega}_{F}(t)} \partial_{t} f+\operatorname{div}\left(f \tilde{u}_{S}\right)=\int_{\tilde{\Omega}_{F}(t)} \partial_{t} f+\tilde{u}_{S} \cdot \nabla f
$$

since $\tilde{u}_{S}$ is divergence free. Thus,

$$
\frac{1}{2} \frac{\mathrm{d}}{\mathrm{d} t} \int_{\tilde{\Omega}_{F}(t)}|\nabla w|^{2}=\int_{\tilde{\Omega}_{F}(t)} \nabla w: \nabla \partial_{t} w+\frac{1}{2} \int_{\tilde{\Omega}_{F}(t)} \tilde{u}_{S} \cdot \nabla|\nabla w|^{2}
$$

Consequently,

$$
\begin{aligned}
I_{11}= & -\frac{1}{2} \int_{0}^{T} \frac{\mathrm{d}}{\mathrm{d} t} \int_{\tilde{\Omega}_{F}(t)}|\nabla w|^{2}-\int_{0}^{T} \int_{\tilde{\Omega}_{F}(t)} \nabla w:\left(\nabla w \nabla \tilde{u}_{S}\right) \\
& -\int_{0}^{T} \int_{\partial \tilde{\Omega}_{S}(t)}(\nabla w n) \cdot\left(\partial_{t} w+\left(\tilde{u}_{S} \cdot \nabla\right) w\right) \\
= & -\int_{0}^{T} \int_{\tilde{\Omega}_{F}(t)} \nabla w:\left(\nabla w \nabla \tilde{u}_{S}\right)-\int_{0}^{T} \int_{\partial \tilde{\Omega}_{S}(t)}(\nabla w n) \cdot\left(\partial_{t} w+\left(\tilde{u}_{S} \cdot \nabla\right) w\right),
\end{aligned}
$$

since $w$ satisfies (2.1). Moreover, according to Lemma 2 and Remark $4, \tilde{u}_{S}$ belongs to $L^{\infty}\left(0, T ; W^{1, \infty}\left(\widetilde{\Omega}_{F}(t)\right)\right)^{2}$ and satisfies

$$
\left\|\tilde{u}_{S}\right\|_{L^{\infty}\left(0, T ; W^{1, \infty}\left(\widetilde{\Omega}_{F}(t)\right)\right)^{2}} \leq C\left(\|\dot{\tilde{a}}\|_{L^{\infty}(0, T)^{2}}+\|\tilde{r}\|_{L^{\infty}(0, T)}\right) \leq C\|\tilde{u}\|_{L^{\infty}((0, T) \times \Omega)} .
$$

The last inequality comes from the fact that $\tilde{u}=\dot{\tilde{a}}+\tilde{r}(x-\tilde{a})^{\perp}$ on $\widetilde{\Omega}_{S}(t)$. Thus, we have

$$
I_{11} \geq-\frac{\widetilde{C}}{s \lambda^{2}} K_{2}-\int_{0}^{T} \int_{\partial \tilde{\Omega}_{S}(t)}(\nabla w n) \cdot\left(\partial_{t} w+\left(\tilde{u}_{S} \cdot \nabla\right) w\right)
$$

where $\widetilde{C}$ depends on $T, \alpha, \beta_{0}$ and $\|\tilde{u}\|_{L^{\infty}((0, T) \times \Omega)}$. We have used that the weight function $\varphi$ is greater than a strictly positive constant which only depends on $T$.

In the sequel of this subsection, we will denote by $\widetilde{C}$ various constants depending on $T, \alpha, \beta_{0}$ and $\|\tilde{u}\|_{L^{\infty}((0, T) \times \Omega)}$ (thus depending on $\|\tilde{a}\|_{W^{1, \infty}(0, T)^{2}}$ and $\left.\|\tilde{r}\|_{L^{\infty}(0, T)}\right)$, and we will denote by $C$ various constants only depending on $T$ and $\beta_{0}$. Integrating by parts, we have, for $I_{12}$,

$$
\begin{aligned}
I_{12}= & -2 s \lambda \int_{0}^{T} \int_{\tilde{\Omega}_{F}(t)} \varphi \Delta w \cdot(\nabla w \nabla \beta) \\
= & s \lambda \int_{0}^{T} \int_{\tilde{\Omega}_{F}(t)} \varphi \nabla|\nabla w|^{2} \cdot \nabla \beta+2 s \lambda \int_{0}^{T} \int_{\tilde{\Omega}_{F}(t)} \nabla w:(\nabla w \nabla(\varphi \nabla \beta)) \\
& +2 s \lambda \int_{0}^{T} \int_{\partial \tilde{\Omega}_{S}(t)} \varphi(\nabla w n) \cdot(\nabla w \nabla \beta)-2 s \lambda \int_{0}^{T} \int_{\partial \Omega} \varphi(\nabla w n) \cdot(\nabla w \nabla \beta) \\
= & -s \lambda \int_{0}^{T} \int_{\tilde{\Omega}_{F}(t)} \varphi|\nabla w|^{2}\left(\Delta \beta+\lambda|\nabla \beta|^{2}\right)+2 s \lambda^{2} \int_{0}^{T} \int_{\tilde{\Omega}_{F}(t)} \varphi|\nabla w \nabla \beta|^{2} \\
& +2 s \lambda \int_{0}^{T} \int_{\tilde{\Omega}_{F}(t)} \varphi \operatorname{tr}\left(\nabla w D^{2} \beta \nabla w^{t}\right)-s \lambda \int_{0}^{T} \int_{\partial \tilde{\Omega}_{S}(t)} \varphi(\nabla \beta \cdot n)|\nabla w|^{2}+s \lambda \int_{0}^{T} \int_{\partial \Omega} \varphi(\nabla \beta \cdot n)|\nabla w|^{2} \\
& +2 s \lambda \int_{0}^{T} \int_{\partial \tilde{\Omega}_{S}(t)} \varphi(\nabla w n) \cdot(\nabla w \nabla \beta)-2 s \lambda \int_{0}^{T} \int_{\partial \Omega} \varphi(\nabla w n) \cdot(\nabla w \nabla \beta),
\end{aligned}
$$


where $D^{2} \beta$ is the matrix $\left(\partial_{i, j}^{2} \beta\right)_{i, j}$. For the boundary terms, we notice that

$$
\begin{aligned}
2(\nabla w n) \cdot(\nabla w \nabla \beta)-(\nabla \beta \cdot n)|\nabla w|^{2}= & 2(\nabla \beta \cdot n)|\nabla w n|^{2}+2(\nabla \beta \cdot \tau)(\nabla w \tau) \cdot(\nabla w n) \\
& -(\nabla \beta \cdot n)|\nabla w n|^{2}-(\nabla \beta \cdot n)|\nabla w \tau|^{2},
\end{aligned}
$$

where $\tau$ is the tangent vector to the boundaries $\partial \widetilde{\Omega}_{S}(t)$ and $\partial \Omega$. Thus, since $\beta$ is zero on the two boundaries, we obtain that

$$
2(\nabla w n) \cdot(\nabla w \nabla \beta)-(\nabla \beta \cdot n)|\nabla w|^{2}=(\nabla \beta \cdot n)\left(|\nabla w n|^{2}-|\nabla w \tau|^{2}\right)
$$

Moreover, on $\partial \Omega, w$ is zero and consequently $\nabla w \tau=0$. On $\partial \widetilde{\Omega}_{S}(t), w$ satisfies $(2.3)$ and $\nabla w \tau=\mathrm{e}^{-s \mathcal{V}^{*}} \gamma n$. Therefore, we have, for $I_{12}$,

$$
\begin{aligned}
I_{12}= & -s \lambda \int_{0}^{T} \int_{\widetilde{\Omega}_{F}(t)} \varphi|\nabla w|^{2}\left(\Delta \beta+\lambda|\nabla \beta|^{2}\right)+2 s \lambda^{2} \int_{0}^{T} \int_{\widetilde{\Omega}_{F}(t)} \varphi|\nabla w \nabla \beta|^{2} \\
& +2 s \lambda \int_{0}^{T} \int_{\widetilde{\Omega}_{F}(t)} \varphi \operatorname{tr}\left(\nabla w D^{2} \beta \nabla w^{t}\right)+s \lambda \int_{0}^{T} \int_{\partial \widetilde{\Omega}_{S}(t)} \varphi(\nabla \beta \cdot n)\left(|\nabla w n|^{2}-\mathrm{e}^{\left.-2 s \mathcal{V}^{*} \gamma^{2}\right)}\right. \\
& -s \lambda \int_{0}^{T} \int_{\partial \Omega} \varphi(\nabla \beta \cdot n)|\nabla w n|^{2} .
\end{aligned}
$$

At last, we obtain:

$$
\begin{aligned}
I_{12} \geq & -s \lambda^{2} \int_{0}^{T} \int_{\widetilde{\Omega}_{F}(t)} \varphi|\nabla \beta|^{2}|\nabla w|^{2}-\frac{\widetilde{C}}{\lambda} K_{2}+s \lambda \int_{0}^{T} \int_{\partial \widetilde{\Omega}_{S}(t)} \varphi(\nabla \beta \cdot n)\left(|\nabla w n|^{2}-\mathrm{e}^{-2 s \mathcal{V}^{*}} \gamma^{2}\right) \\
& -s \lambda \int_{0}^{T} \int_{\partial \Omega} \varphi(\nabla \beta \cdot n)|\nabla w n|^{2}, \\
\geq & -s \lambda^{2} \int_{0}^{T} \int_{\widetilde{\Omega}_{F}(t)} \varphi|\nabla \beta|^{2}|\nabla w|^{2}-\frac{\widetilde{C}}{\lambda} K_{2}+s \lambda \int_{0}^{T} \int_{\partial \widetilde{\Omega}_{S}(t)} \varphi(\nabla \beta \cdot n)\left(|\nabla w n|^{2}-\mathrm{e}^{-2 s \mathcal{V}^{*}} \gamma^{2}\right),
\end{aligned}
$$

according to properties (1.26) and (1.25) satisfied by $\beta$. We consider

$$
\begin{aligned}
I_{13}= & -2 s \lambda^{2} \int_{0}^{T} \int_{\widetilde{\Omega}_{F}(t)} \varphi|\nabla \beta|^{2} \Delta w \cdot w \\
= & 2 s \lambda^{2} \int_{0}^{T} \int_{\widetilde{\Omega}_{F}(t)} \varphi|\nabla \beta|^{2}|\nabla w|^{2}+2 s \lambda^{2} \int_{0}^{T} \int_{\widetilde{\Omega}_{F}(t)} \varphi\left(\nabla w \nabla\left(|\nabla \beta|^{2}\right)\right) \cdot w \\
& +2 s \lambda^{3} \int_{0}^{T} \int_{\widetilde{\Omega}_{F}(t)} \varphi|\nabla \beta|^{2}(\nabla w \nabla \beta) \cdot w+2 s \lambda^{2} \int_{0}^{T} \int_{\partial \widetilde{\Omega}_{S}(t)} \varphi|\nabla \beta|^{2}(\nabla w n) \cdot w .
\end{aligned}
$$

Therefore,

$$
I_{13} \geq 2 s \lambda^{2} \int_{0}^{T} \int_{\widetilde{\Omega}_{F}(t)} \varphi|\nabla \beta|^{2}|\nabla w|^{2}-\frac{\widetilde{C}}{s} K_{1}-\frac{\widetilde{C}}{s} K_{2}+2 s \lambda^{2} \int_{0}^{T} \int_{\partial \widetilde{\Omega}_{S}(t)} \varphi|\nabla \beta|^{2}(\nabla w n) \cdot w
$$


This inequality is obtained thanks to Cauchy-Schwarz inequality. We also define

$$
\begin{aligned}
I_{21}= & s^{2} \lambda^{2} \int_{0}^{T} \int_{\tilde{\Omega}_{F}(t)} \varphi^{2}|\nabla \beta|^{2} w \cdot\left(\partial_{t} w+\left(\tilde{u}_{S} \cdot \nabla\right) w\right) \\
= & \frac{1}{2} s^{2} \lambda^{2} \int_{0}^{T} \int_{\tilde{\Omega}_{F}(t)} \varphi^{2}|\nabla \beta|^{2}\left(\partial_{t}|w|^{2}+\left(\tilde{u}_{S} \cdot \nabla\right)|w|^{2}\right) \\
= & -\frac{1}{2} s^{2} \lambda^{2} \int_{0}^{T} \int_{\tilde{\Omega}_{F}(t)}|w|^{2}\left(\partial_{t}\left(\varphi^{2}|\nabla \beta|^{2}\right)+\tilde{u}_{S} \cdot \nabla\left(\varphi^{2}|\nabla \beta|^{2}\right)\right) \\
= & -s^{2} \lambda^{2} \int_{0}^{T} \int_{\tilde{\Omega}_{F}(t)} \varphi|\nabla \beta|^{2}|w|^{2}\left(\partial_{t} \varphi+\tilde{u}_{S} \cdot \nabla \varphi\right) \\
& -s^{2} \lambda^{2} \int_{0}^{T} \int_{\tilde{\Omega}_{F}(t)} \varphi^{2}|w|^{2} \nabla \beta \cdot\left(\partial_{t} \nabla \beta+\left(\tilde{u}_{S} \cdot \nabla\right) \nabla \beta\right),
\end{aligned}
$$

thanks to formula (2.9) and property (2.1). We obtain:

$$
I_{21} \geq-\frac{\widetilde{C}}{s \lambda} K_{1}
$$

Next, we have

$$
\begin{aligned}
I_{22}= & -2 s^{3} \lambda^{3} \int_{0}^{T} \int_{\tilde{\Omega}_{F}(t)} \varphi^{3}|\nabla \beta|^{2} w \cdot(\nabla w \nabla \beta)=-s^{3} \lambda^{3} \int_{0}^{T} \int_{\tilde{\Omega}_{F}(t)} \varphi^{3}|\nabla \beta|^{2} \nabla|w|^{2} \cdot \nabla \beta \\
= & s^{3} \lambda^{3} \int_{0}^{T} \int_{\tilde{\Omega}_{F}(t)}|w|^{2} \operatorname{div}\left(\varphi^{3}|\nabla \beta|^{2} \nabla \beta\right)+s^{3} \lambda^{3} \int_{0}^{T} \int_{\partial \tilde{\Omega}_{S}(t)} \varphi^{3}|\nabla \beta|^{2}|w|^{2} \nabla \beta \cdot n \\
= & 3 s^{3} \lambda^{4} \int_{0}^{T} \int_{\tilde{\Omega}_{F}(t)} \varphi^{3}|\nabla \beta|^{4}|w|^{2}+s^{3} \lambda^{3} \int_{0}^{T} \int_{\tilde{\Omega}_{F}(t)} \varphi^{3}|w|^{2} \operatorname{div}\left(|\nabla \beta|^{2} \nabla \beta\right) \\
& +s^{3} \lambda^{3} \int_{0}^{T} \int_{\partial \tilde{\Omega}_{S}(t)} \varphi^{3}|\nabla \beta|^{2}|w|^{2} \nabla \beta \cdot n .
\end{aligned}
$$

Therefore,

$$
I_{22} \geq 3 s^{3} \lambda^{4} \int_{0}^{T} \int_{\widetilde{\Omega}_{F}(t)} \varphi^{3}|\nabla \beta|^{4}|w|^{2}-\frac{\widetilde{C}}{\lambda} K_{1}+s^{3} \lambda^{3} \int_{0}^{T} \int_{\partial \tilde{\Omega}_{S}(t)} \varphi^{3}|\nabla \beta|^{2}|w|^{2} \nabla \beta \cdot n .
$$

At last, we have

$$
I_{23}=-2 s^{3} \lambda^{4} \int_{0}^{T} \int_{\tilde{\Omega}_{F}(t)} \varphi^{3}|\nabla \beta|^{4}|w|^{2} .
$$


Reassembling all these inequalities, we conclude that

$$
\begin{gathered}
\left(L_{1}(w), L_{2}(w)\right)_{2} \geq s^{3} \lambda^{4} \int_{0}^{T} \int_{\widetilde{\Omega}_{F}(t)} \varphi^{3}|\nabla \beta|^{4}|w|^{2}+s \lambda^{2} \int_{0}^{T} \int_{\widetilde{\Omega}_{F}(t)} \varphi|\nabla \beta|^{2}|\nabla w|^{2} \\
+s^{3} \lambda^{3} \int_{0}^{T} \int_{\partial \widetilde{\Omega}_{S}(t)} \varphi^{3}|\nabla \beta|^{2}|w|^{2} \nabla \beta \cdot n+s \lambda \int_{0}^{T} \int_{\partial \widetilde{\Omega}_{S}(t)} \varphi(\nabla \beta \cdot n)|\nabla w n|^{2}-\widetilde{C}\left(\frac{1}{s}+\frac{1}{\lambda}\right) K_{1} \\
-\widetilde{C}\left(\frac{1}{s}+\frac{1}{\lambda}\right) K_{2}-s \lambda \int_{0}^{T} \int_{\partial \widetilde{\Omega}_{S}(t)} \mathrm{e}^{-2 s \mathcal{V}^{*}} \varphi(\nabla \beta \cdot n) \gamma^{2} \\
+2 s \lambda^{2} \int_{0}^{T} \int_{\partial \widetilde{\Omega}_{S}(t)} \varphi|\nabla \beta|^{2}(\nabla w n) \cdot w-\int_{0}^{T} \int_{\partial \widetilde{\Omega}_{S}(t)}(\nabla w n) \cdot\left(\partial_{t} w+\left(\tilde{u}_{S} \cdot \nabla\right) w\right) .
\end{gathered}
$$

As we will see later, the last term, which comes from the fluid-structure interaction, will be bounded thanks to the solid equations. The last hypothesis of (1.9) is important at this step of the proof to deduce, from estimates on $w$ on the boundary, estimates on the structure motion. Indeed, we have

$$
\int_{\partial \widetilde{\Omega}_{S}(t)}|w|^{2}=\mathrm{e}^{-2 s \mathcal{V}^{*}} \int_{\partial \widetilde{\Omega}_{S}(t)}\left|\dot{b}+\gamma(x-\tilde{a})^{\perp}\right|^{2} .
$$

Since condition (1.9) is prescribed, if we develop this expression, we notice that the scalar product is equal to 0. Thus, we obtain

$$
\int_{\partial \widetilde{\Omega}_{S}(t)}|w|^{2}=\mathrm{e}^{-2 s \mathcal{V}^{*}} \int_{\partial \widetilde{\Omega}_{S}(t)}|\dot{b}|^{2}+\mathrm{e}^{-2 s \mathcal{V}^{*}} \int_{\partial \widetilde{\Omega}_{S}(t)}\left|\gamma(x-\tilde{a})^{\perp}\right|^{2} .
$$

Moreover, since

$$
\int_{\partial \widetilde{\Omega}_{S}(t)}\left|(x-\tilde{a})^{\perp}\right|^{2}=\int_{\partial \widetilde{\Omega}_{S}(t)}|x-\tilde{a}|^{2}=\int_{\partial \Omega_{S}(0)}\left|y-a_{0}\right|^{2},
$$

we deduce that

$$
\mathrm{e}^{-2 s \mathcal{V}^{*}}\left(|\dot{b}|^{2}+\gamma^{2}\right) \leq C \int_{\partial \widetilde{\Omega}_{S}(t)}|w|^{2}
$$

Therefore,

$$
s \lambda \int_{0}^{T} \int_{\partial \widetilde{\Omega}_{S}(t)} \mathrm{e}^{-2 s \mathcal{V}^{*}} \varphi(\nabla \beta \cdot n) \gamma^{2} \leq C s \lambda \int_{0}^{T} \int_{\partial \widetilde{\Omega}_{S}(t)} \varphi^{3}|\nabla \beta|^{2}|w|^{2} \nabla \beta \cdot n .
$$

Here, we have used that $\nabla \beta \cdot n \geq c_{2}>0$ on $\partial \widetilde{\Omega}_{S}(t)$. Thus, for $s \geq s_{0}$ and $\lambda \geq \lambda_{0}$, where $s_{0}$ and $\lambda_{0}$ depend on $T, \alpha, \beta_{0}$ and the norm of $\tilde{u}$ in $L^{\infty}((0, T) \times \Omega)$, equation $(2.8)$ becomes:

$$
\begin{gathered}
\left\|L_{1}(w)\right\|_{2}^{2}+\left\|L_{2}(w)\right\|_{2}^{2}+2 s^{3} \lambda^{4} \int_{0}^{T} \int_{\widetilde{\Omega}_{F}(t)} \varphi^{3}|\nabla \beta|^{4}|w|^{2}+2 s \lambda^{2} \int_{0}^{T} \int_{\widetilde{\Omega}_{F}(t)} \varphi|\nabla \beta|^{2}|\nabla w|^{2} \\
-\widetilde{C}\left(\frac{1}{s}+\frac{1}{\lambda}\right) K_{1}-\widetilde{C}\left(\frac{1}{s}+\frac{1}{\lambda}\right) K_{2}+s^{3} \lambda^{3} \int_{0}^{T} \int_{\partial \widetilde{\Omega}_{S}(t)} \varphi^{3}|\nabla \beta|^{2}|w|^{2} \nabla \beta \cdot n \\
+2 s \lambda \int_{0}^{T} \int_{\partial \widetilde{\Omega}_{S}(t)} \varphi(\nabla \beta \cdot n)|\nabla w n|^{2}-2 \int_{0}^{T} \int_{\partial \widetilde{\Omega}_{S}(t)}(\nabla w n) \cdot\left(\partial_{t} w+\left(\tilde{u}_{S} \cdot \nabla\right) w\right) \\
\leq\left\|g_{s}\right\|_{2}^{2}-4 s \lambda^{2} \int_{0}^{T} \int_{\partial \widetilde{\Omega}_{S}(t)} \varphi|\nabla \beta|^{2}(\nabla w n) \cdot w .
\end{gathered}
$$


Now, we notice, according to the definition (2.6) of $L_{1}$, that

$$
\int_{0}^{T} \int_{\widetilde{\Omega}_{F}(t)} \frac{1}{s \varphi}|\Delta w|^{2} \leq C \int_{0}^{T} \int_{\widetilde{\Omega}_{F}(t)} \frac{1}{s \varphi}\left|L_{1}(w)\right|^{2}+C s^{3} \lambda^{4} \int_{0}^{T} \int_{\widetilde{\Omega}_{F}(t)} \varphi^{3}|\nabla \beta|^{4}|w|^{2} .
$$

In the same way, according to the definition (2.7) of $L_{2}$, we have

$$
\int_{0}^{T} \int_{\widetilde{\Omega}_{F}(t)} \frac{1}{s \varphi}\left|\partial_{t} w\right|^{2} \leq C \int_{0}^{T} \int_{\widetilde{\Omega}_{F}(t)} \frac{1}{s \varphi}\left|L_{2}(w)\right|^{2}+\frac{\widetilde{C}}{s^{2}} K_{1}+\frac{\widetilde{C}}{s} K_{2}+C s \lambda^{2} \int_{0}^{T} \int_{\widetilde{\Omega}_{F}(t)} \varphi|\nabla \beta|^{2}|\nabla w|^{2} .
$$

Moreover, we can write that

$$
\left\|g_{s}\right\|_{2}^{2} \leq \frac{\widetilde{C}}{s} K_{1}+\frac{\widetilde{C}}{s \lambda^{2}} K_{2}+C \int_{0}^{T} \int_{\widetilde{\Omega}_{F}(t)} \mathrm{e}^{-2 s \mathcal{V}}|\nabla q|^{2}
$$

Indeed, since the definition (1.27) of $\mathcal{V}$ involves $t(T-t)$ to the power 4 at the denominator, we have

$$
\left|\partial_{t} \mathcal{V}+\tilde{u} \cdot \nabla \mathcal{V}\right| \leq \widetilde{C} \lambda \varphi^{5 / 4}
$$

We also notice that

$$
-4 s \lambda^{2} \int_{0}^{T} \int_{\partial \widetilde{\Omega}_{S}(t)} \varphi|\nabla \beta|^{2}(\nabla w n) \cdot w \leq C \int_{0}^{T} \int_{\partial \widetilde{\Omega}_{S}(t)} \lambda|\nabla w n|^{2}+s^{2} \lambda^{3} \varphi^{2}|w|^{2},
$$

and

$$
\int_{0}^{T} \int_{\partial \widetilde{\Omega}_{S}(t)}(\nabla w n) \cdot\left(\partial_{t} w+\left(\tilde{u}_{S} \cdot \nabla\right) w\right) \leq C \int_{0}^{T} \int_{\partial \tilde{\Omega}_{S}(t)}|\nabla w n|^{2}+\left|\partial_{t} w+\left(\tilde{u}_{S} \cdot \nabla\right) w\right|^{2}
$$

At last, according to the property (1.25) satisfied by $\beta$, we have

$$
K_{1} \leq \widetilde{C} s^{3} \lambda^{4} \int_{0}^{T} \int_{\widetilde{\Omega}_{F}(t)} \varphi^{3}|\nabla \beta|^{4}|w|^{2}+s^{3} \lambda^{4} \int_{0}^{T} \int_{\omega_{0}} \varphi^{3}|w|^{2}
$$

and

$$
K_{2} \leq \widetilde{C} s \lambda^{2} \int_{0}^{T} \int_{\widetilde{\Omega}_{F}(t)} \varphi|\nabla \beta|^{2}|\nabla w|^{2}+s \lambda^{2} \int_{0}^{T} \int_{\omega_{0}} \varphi|\nabla w|^{2}
$$

Therefore, we obtain, for $s \geq s_{1}$ and $\lambda \geq \lambda_{1}$ where $s_{1}$ and $\lambda_{1}$ depends on $T, \alpha, \beta_{0}$ and the norm of $\tilde{u}$ in $L^{\infty}((0, T) \times \Omega):$

$$
\begin{gathered}
\int_{0}^{T} \int_{\widetilde{\Omega}_{F}(t)} \frac{1}{s \varphi}|\Delta w|^{2}+\int_{0}^{T} \int_{\widetilde{\Omega}_{F}(t)} \frac{1}{s \varphi}\left|\partial_{t} w\right|^{2}+s^{3} \lambda^{4} \int_{0}^{T} \int_{\widetilde{\Omega}_{F}(t)} \varphi^{3}|w|^{2} \\
+s \lambda^{2} \int_{0}^{T} \int_{\widetilde{\Omega}_{F}(t)} \varphi|\nabla w|^{2}+s^{3} \lambda^{3} \int_{0}^{T} \int_{\partial \widetilde{\Omega}_{S}(t)} \varphi^{3}|w|^{2}+s \lambda \int_{0}^{T} \int_{\partial \widetilde{\Omega}_{S}(t)} \varphi|\nabla w n|^{2} \\
\leq \widetilde{C}\left(\int_{0}^{T} \int_{\partial \widetilde{\Omega}_{S}(t)}\left|\partial_{t} w+\left(\tilde{u}_{S} \cdot \nabla\right) w\right|^{2}+\int_{0}^{T} \int_{\widetilde{\Omega}_{F}(t)} \mathrm{e}^{-2 s \mathcal{V}}|\nabla q|^{2}\right. \\
\left.+\int_{0}^{T} \int_{\omega_{0}}\left(s^{3} \lambda^{4} \varphi^{3}|w|^{2}+s \lambda^{2} \varphi|\nabla w|^{2}\right)\right)
\end{gathered}
$$


Now, we come back to our initial variable $v$. Thanks to (2.4) and (2.5), we obtain the following estimates:

$$
\begin{aligned}
& s \lambda^{2} \int_{0}^{T} \int_{\widetilde{\Omega}_{F}(t)} \mathrm{e}^{-2 s \mathcal{V}} \varphi|\nabla v|^{2} \leq \widetilde{C}\left(s \lambda^{2} \int_{0}^{T} \int_{\widetilde{\Omega}_{F}(t)} \varphi|\nabla w|^{2}+s^{3} \lambda^{4} \int_{0}^{T} \int_{\widetilde{\Omega}_{F}(t)} \varphi^{3}|w|^{2}\right), \\
& \int_{0}^{T} \int_{\widetilde{\Omega}_{F}(t)} \frac{\mathrm{e}^{-2 s \mathcal{V}}}{s \varphi}|\Delta v|^{2} \leq \widetilde{C}\left(\int_{0}^{T} \int_{\widetilde{\Omega}_{F}(t)} \frac{1}{s \varphi}|\Delta w|^{2}+s \lambda^{2} \int_{0}^{T} \int_{\widetilde{\Omega}_{F}(t)} \varphi|\nabla w|^{2}\right. \\
& \\
&\left.\quad+s \lambda^{2} \int_{0}^{T} \int_{\widetilde{\Omega}_{F}(t)} \varphi|w|^{2}+s^{3} \lambda^{4} \int_{0}^{T} \int_{\widetilde{\Omega}_{F}(t)} \varphi^{3}|w|^{2}\right), \\
& \int_{0}^{T} \int_{\widetilde{\Omega}_{F}(t)} \frac{\mathrm{e}^{-2 s \mathcal{V}}}{s \varphi}\left|\partial_{t} v\right|^{2} \leq \widetilde{C}\left(\int_{0}^{T} \int_{\widetilde{\Omega}_{F}(t)} \frac{1}{s \varphi}\left|\partial_{t} w\right|^{2}+s \lambda^{2} \int_{0}^{T} \int_{\widetilde{\Omega}_{F}(t)} \varphi^{3}|w|^{2}\right) \\
& s \lambda \int_{0}^{T} \int_{\partial \widetilde{\Omega}_{S}(t)} \mathrm{e}^{-2 s \mathcal{V}} \varphi|\nabla v n|^{2} \leq C s \lambda \int_{0}^{T} \int_{\partial \widetilde{\Omega}_{S}(t)}|\nabla w n|^{2}+C s^{3} \lambda^{3} \int_{0}^{T} \int_{\partial \widetilde{\Omega}_{S}(t)} \varphi^{3}|w|^{2} .
\end{aligned}
$$

Moreover, since

$$
\partial_{t} w+\left(\tilde{u}_{S} \cdot \nabla\right) w=\mathrm{e}^{-s \mathcal{V}}\left(\partial_{t} v+\left(\tilde{u}_{S} \cdot \nabla\right) v-s\left(\mathrm{e}^{10 \lambda M}-\mathrm{e}^{\lambda(8 M+\beta)}\right) \frac{\mathrm{d}}{\mathrm{d} t}\left(\frac{1}{t^{4}(T-t)^{4}}\right) v\right)
$$

we have

$$
\int_{\partial \widetilde{\Omega}_{S}(t)}\left|\partial_{t} w+\left(\tilde{u}_{S} \cdot \nabla\right) w\right|^{2} \leq C\left(\int_{\partial \widetilde{\Omega}_{S}(t)} \mathrm{e}^{-2 s \mathcal{V}}\left|\partial_{t} v+\left(\tilde{u}_{S} \cdot \nabla\right) v\right|^{2}+s^{2} \int_{\partial \widetilde{\Omega}_{S}(t)} \mathrm{e}^{-2 s \mathcal{V}} \varphi^{3}|v|^{2}\right) .
$$

Finally, we obtain, for $s \geq s_{2}$ and $\lambda \geq \lambda_{2}$ where $s_{2}$ and $\lambda_{2}$ only depend on $T, \alpha, \beta_{0}$ and the norm of $\tilde{u}$ in $L^{\infty}((0, T) \times \Omega)$,

$$
\begin{aligned}
& \int_{0}^{T} \int_{\widetilde{\Omega}_{F}(t)} \mathrm{e}^{-2 s \mathcal{V}}\left(\frac{1}{s \varphi}\left(|\Delta v|^{2}+\left|\partial_{t} v\right|^{2}\right)+s \lambda^{2} \varphi|\nabla v|^{2}+s^{3} \lambda^{4} \varphi^{3}|v|^{2}\right) \\
& +s^{3} \lambda^{3} \int_{0}^{T} \int_{\partial \widetilde{\Omega}_{S}(t)} \mathrm{e}^{-2 s \mathcal{V}} \varphi^{3}|v|^{2}+s \lambda \int_{0}^{T} \int_{\partial \widetilde{\Omega}_{S}(t)} \mathrm{e}^{-2 s \mathcal{V}} \varphi|\nabla v n|^{2} \\
& \leq \widetilde{C}\left(\int_{0}^{T} \int_{\partial \widetilde{\Omega}_{S}(t)} \mathrm{e}^{-2 s \mathcal{V}}\left|\partial_{t} v+\left(\tilde{u}_{S} \cdot \nabla\right) v\right|^{2}+\int_{0}^{T} \int_{\widetilde{\Omega}_{F}(t)} \mathrm{e}^{-2 s \mathcal{V}}|\nabla q|^{2}\right. \\
& \left.+\int_{0}^{T} \int_{\omega_{0}} \mathrm{e}^{-2 s \mathcal{V}}\left(s^{3} \lambda^{4} \varphi^{3}|v|^{2}+s \lambda^{2} \varphi|\nabla v|^{2}\right)\right) .
\end{aligned}
$$

Thanks to the equations of the structure motion, we are able to obtain estimates on the acceleration of the motion. Indeed, we deduce from the second and third equations of system (1.22) that

$$
s \lambda \int_{0}^{T} \mathrm{e}^{-2 s \mathcal{V}^{*}} \varphi^{*}\left(|\ddot{b}|^{2}+|\dot{\gamma}|^{2}\right) \leq s \lambda \int_{0}^{T} \int_{\partial \widetilde{\Omega}_{S}(t)} \mathrm{e}^{-2 s \mathcal{V}} \varphi|\nabla v|^{2}+s \lambda \int_{0}^{T} \int_{\partial \widetilde{\Omega}_{S}(t)} \mathrm{e}^{-2 s \mathcal{V}} \varphi|q|^{2} .
$$


Now, we have

$$
\begin{aligned}
\int_{0}^{T} \int_{\partial \tilde{\Omega}_{S}(t)} \mathrm{e}^{-2 s \mathcal{V}} \varphi|\nabla v|^{2} & \leq \int_{0}^{T} \int_{\partial \widetilde{\Omega}_{S}(t)} \mathrm{e}^{-2 s \mathcal{V}} \varphi\left(|\nabla v n|^{2}+|\nabla v \tau|^{2}\right) \\
& \leq C \int_{0}^{T} \int_{\partial \widetilde{\Omega}_{S}(t)} \mathrm{e}^{-2 s \mathcal{V}} \varphi\left(|\nabla v n|^{2}+|v|^{2}\right)
\end{aligned}
$$

since on $\partial \widetilde{\Omega}_{S}(t), \nabla v \tau=\gamma n$ and since (2.10) is satisfied. This last inequality is very strong and holds in our problem since the fluid velocity has a specific writing. Thus, we obtain for $s \geq s_{3}$ and $\lambda \geq \lambda_{3}$ where $s_{3}$ and $\lambda_{3}$ only depend on $T, \alpha, \beta_{0}$ and the norm of $\tilde{u}$ in $L^{\infty}((0, T) \times \Omega)$,

$$
\begin{aligned}
& \int_{0}^{T} \int_{\widetilde{\Omega}_{F}(t)} \mathrm{e}^{-2 s \mathcal{V}}\left(\frac{1}{s \varphi}\left(|\Delta v|^{2}+\left|\partial_{t} v\right|^{2}\right)+s \lambda^{2} \varphi|\nabla v|^{2}+s^{3} \lambda^{4} \varphi^{3}|v|^{2}\right)+s \lambda \int_{0}^{T} \mathrm{e}^{-2 s \mathcal{V}^{*}} \varphi^{*}|\ddot{b}|^{2} \\
& s \lambda \int_{0}^{T} \mathrm{e}^{-2 s \mathcal{V}^{*}} \varphi^{*}|\dot{\gamma}|^{2}+s^{3} \lambda^{3} \int_{0}^{T} \int_{\partial \widetilde{\Omega}_{S}(t)} \mathrm{e}^{-2 s \mathcal{V}} \varphi^{3}|v|^{2}+s \lambda \int_{0}^{T} \int_{\partial \widetilde{\Omega}_{S}(t)} \mathrm{e}^{-2 s \mathcal{V}} \varphi|\nabla v n|^{2} \\
& \leq \widetilde{C}\left(\int_{0}^{T} \int_{\partial \widetilde{\Omega}_{S}(t)} \mathrm{e}^{-2 s \mathcal{V}}\left|\partial_{t} v+\left(\tilde{u}_{S} \cdot \nabla\right) v\right|^{2}+s \lambda \int_{0}^{T} \int_{\partial \widetilde{\Omega}_{S}(t)} \mathrm{e}^{-2 s \mathcal{V}} \varphi|q|^{2}+\int_{0}^{T} \int_{\widetilde{\Omega}_{F}(t)} \mathrm{e}^{-2 s \mathcal{V}}|\nabla q|^{2}\right. \\
& \left.+\int_{0}^{T} \int_{\omega_{0}} \mathrm{e}^{-2 s \mathcal{V}}\left(s^{3} \lambda^{4} \varphi^{3}|v|^{2}+s \lambda^{2} \varphi|\nabla v|^{2}\right)\right) .
\end{aligned}
$$

Next, we notice that, on $\partial \widetilde{\Omega}_{S}(t)$

$$
\partial_{t} v+\left(\tilde{u}_{S} \cdot \nabla\right) v=\ddot{b}+\dot{\gamma}(x-\tilde{a})^{\perp}-\gamma \tilde{r}(x-\tilde{a}) .
$$

Thus, we finally get that, for $s \geq s_{4}$ and $\lambda \geq \lambda_{4}$ where $s_{4}$ and $\lambda_{4}$ only depend on $T, \alpha, \beta_{0}$ and the norm of $\tilde{u}$ in $L^{\infty}((0, T) \times \Omega)$,

$$
\begin{aligned}
& \int_{0}^{T} \int_{\widetilde{\Omega}_{F}(t)} \mathrm{e}^{-2 s \mathcal{V}}\left(\frac{1}{s \varphi}\left(|\Delta v|^{2}+\left|\partial_{t} v\right|^{2}\right)+s \lambda^{2} \varphi|\nabla v|^{2}+s^{3} \lambda^{4} \varphi^{3}|v|^{2}\right)+s \lambda \int_{0}^{T} \mathrm{e}^{-2 s \mathcal{V}^{*}} \varphi^{*}|\ddot{b}|^{2} \\
& s \lambda \int_{0}^{T} \mathrm{e}^{-2 s \mathcal{V}^{*}} \varphi^{*}|\dot{\gamma}|^{2}+s^{3} \lambda^{3} \int_{0}^{T} \int_{\partial \widetilde{\Omega}_{S}(t)} \mathrm{e}^{-2 s \mathcal{V}} \varphi^{3}|v|^{2}+s \lambda \int_{0}^{T} \int_{\partial \widetilde{\Omega}_{S}(t)} \mathrm{e}^{-2 s \mathcal{V}} \varphi|\nabla v n|^{2} \\
& \leq \widetilde{C}\left(s \lambda \int_{0}^{T} \int_{\partial \widetilde{\Omega}_{S}(t)} \mathrm{e}^{-2 s \mathcal{V}} \varphi|q|^{2}+\int_{0}^{T} \int_{\widetilde{\Omega}_{F}(t)} \mathrm{e}^{-2 s \mathcal{V}}|\nabla q|^{2}\right. \\
& \left.+\int_{0}^{T} \int_{\omega_{0}} \mathrm{e}^{-2 s \mathcal{V}}\left(s^{3} \lambda^{4} \varphi^{3}|v|^{2}+s \lambda^{2} \varphi|\nabla v|^{2}\right)\right) .
\end{aligned}
$$

We recall that, in order to estimate the last term in (2.11), we have used (2.10).

\subsection{Estimate on the pressure}

We now want to obtain a bound on the two integrals in $q$ in the right-hand side of this expression in terms of a local integral of $q$. Applying the divergence operator to the first equation of (1.22), we obtain, for almost every $t \in(0, T)$,

$$
\Delta q(t)=\operatorname{div}((\tilde{u} \cdot \nabla) v)(t) \text { in } \widetilde{\Omega}_{F}(t)
$$


Here, the capital point is to apply to this elliptic problem defined on the regular domain $\widetilde{\Omega}_{F}(t)$ the Carleman inequality obtained in [13]. It allows to assert that, for almost every $t \in(0, T)$, there exists a constant $C$ depending on $\Omega$ and $\omega$, and two real numbers $\bar{\lambda}$ and $\bar{\tau}$ such that, for all $\lambda \geq \bar{\lambda}$ and $\tau \geq \bar{\tau}$, we have

$$
\begin{gathered}
\tau^{2} \lambda^{2} \int_{\widetilde{\Omega}_{F}(t)} \mathrm{e}^{2 \tau \psi(t)} \psi(t)^{2}|q(t)|^{2}+\int_{\widetilde{\Omega}_{F}(t)} \mathrm{e}^{2 \tau \psi(t)}|\nabla q(t)|^{2} \leq C\left(\tau \int_{\widetilde{\Omega}_{F}(t)} \mathrm{e}^{2 \tau \psi(t)} \psi(t)|(\tilde{u} \cdot \nabla) v|^{2}(t)\right. \\
\left.+\sqrt{\tau} \mathrm{e}^{2 \tau}\|q(t)\|_{H^{1 / 2}\left(\partial \Omega \cup \partial \widetilde{\Omega}_{S}(t)\right)}^{2}+\int_{\omega_{0}} \mathrm{e}^{2 \tau \psi(t)}\left(|\nabla q(t)|^{2}+\tau^{2} \lambda^{2} \psi(t)^{2}|q(t)|^{2}\right)\right),
\end{gathered}
$$

where $\psi$ is defined by

$$
\psi(t)=\mathrm{e}^{\lambda \beta(t, \cdot)} \text { in } \widetilde{\Omega}_{F}(t), \text { for all } t \in(0, T) .
$$

Next, proceeding as in [10] (we refer to this paper for complementary explanations and computations), we eliminate the local integral in $\nabla q$ in the right-hand side by integrating by parts several times and using (2.13). If we consider an open set $\omega_{1}$ such that $\omega_{0} \subset \subset \omega_{1} \subset \subset \omega$, we get

$$
\int_{\omega_{0}} \mathrm{e}^{2 \tau \psi(t)}|\nabla q(t)|^{2} \leq C\left(\tau^{2} \lambda^{2} \int_{\omega_{1}} \mathrm{e}^{2 \tau \psi(t)} \psi(t)^{2}|q(t)|^{2}+\int_{\omega_{1}} \mathrm{e}^{2 \tau \psi(t)}|(\tilde{u} \cdot \nabla) v|^{2}(t)\right)
$$

Thus, we have

$$
\begin{gathered}
\tau^{2} \lambda^{2} \int_{\widetilde{\Omega}_{F}(t)} \mathrm{e}^{2 \tau \psi(t)} \psi(t)^{2}|q(t)|^{2}+\int_{\widetilde{\Omega}_{F}(t)} \mathrm{e}^{2 \tau \psi(t)}|\nabla q(t)|^{2} \\
\leq C\left(\tau \int_{\widetilde{\Omega}_{F}(t)} \mathrm{e}^{2 \tau \psi(t)} \psi(t)|(\tilde{u} \cdot \nabla) v|^{2}(t)+\sqrt{\tau} \mathrm{e}^{2 \tau}\|q(t)\|_{H^{1 / 2}\left(\partial \Omega \cup \partial \widetilde{\Omega}_{S}(t)\right)}^{2}\right. \\
\left.+\tau^{2} \lambda^{2} \int_{\omega_{1}} \mathrm{e}^{2 \tau \psi(t)} \psi^{2}|q(t)|^{2}\right)
\end{gathered}
$$

To use this estimate in $(2.12)$, we see that we have to take $\tau=\frac{s \mathrm{e}^{8 \lambda M}}{t^{4}(T-t)^{4}}$. Next, we multiply this equation by

$$
\exp \left(-2 s \frac{\mathrm{e}^{10 \lambda M}}{t^{4}(T-t)^{4}}\right)
$$

and we integrate in time. In this way, we obtain that

$$
\begin{aligned}
& s^{2} \lambda^{2} \int_{0}^{T} \int_{\widetilde{\Omega}_{F}(t)} \mathrm{e}^{-2 s \mathcal{V}} \varphi^{2}|q|^{2}+\int_{0}^{T} \int_{\widetilde{\Omega}_{F}(t)} \mathrm{e}^{-2 s \mathcal{V}}|\nabla q|^{2} \leq C\left(s \int_{0}^{T} \int_{\widetilde{\Omega}_{F}(t)} \mathrm{e}^{-2 s \mathcal{V}} \varphi|(\tilde{u} \cdot \nabla) v|^{2}\right. \\
& \left.\quad+s^{1 / 2} \int_{0}^{T} \mathrm{e}^{-2 s \mathcal{V}^{*}}\left(\varphi^{*}\right)^{1 / 2}\|q(t)\|_{H^{1 / 2}\left(\partial \Omega \cup \partial \widetilde{\Omega}_{S}(t)\right)}^{2}+s^{2} \lambda^{2} \int_{0}^{T} \int_{\omega_{1}} \mathrm{e}^{-2 s \mathcal{V}} \varphi^{2}|q|^{2}\right),
\end{aligned}
$$

where $\varphi^{*}$ and $\mathcal{V}^{*}$ are defined in (1.28) and (1.29). Moreover, since the boundary term satisfies

$$
s \lambda \int_{0}^{T} \int_{\partial \widetilde{\Omega}_{S}(t)} \mathrm{e}^{-2 s \mathcal{V}} \varphi|q|^{2} \leq C \int_{0}^{T} \int_{\widetilde{\Omega}_{S}(t)}\left(s^{2} \lambda^{2} \varphi^{2}\left|\mathrm{e}^{-s \mathcal{V}} q\right|^{2}+\left|\nabla\left(\mathrm{e}^{-s \mathcal{V}} q\right)\right|^{2}\right)
$$

it is bounded by the terms in the right-hand side of (2.14). Therefore, for the two terms in $q$ in the right-hand side of (2.12), we have, for $s \geq s_{5}$ and $\lambda \geq \lambda_{5}$ where $s_{5}$ and $\lambda_{5}$ only depend on $T, \alpha, \beta_{0}$ and the norm of $\tilde{u}$ in 
$L^{\infty}((0, T) \times \Omega)$,

$$
\begin{aligned}
& s \lambda \int_{0}^{T} \int_{\partial \tilde{\Omega}_{S}(t)} \mathrm{e}^{-2 s \mathcal{V}} \varphi|q|^{2}+\int_{0}^{T} \int_{\tilde{\Omega}_{F}(t)} \mathrm{e}^{-2 s \mathcal{V}}|\nabla q|^{2} \leq C\left(s \int_{0}^{T} \int_{\tilde{\Omega}_{F}(t)} \mathrm{e}^{-2 s \mathcal{V}} \varphi|(\tilde{u} \cdot \nabla) v|^{2}\right. \\
& \left.\quad+s^{1 / 2} \int_{0}^{T} \mathrm{e}^{-2 s \mathcal{V}^{*}}\left(\varphi^{*}\right)^{1 / 2}\|q(t)\|_{H^{1 / 2}\left(\partial \Omega \cup \partial \tilde{\Omega}_{S}(t)\right)}^{2}+s^{2} \lambda^{2} \int_{0}^{T} \int_{\omega_{1}} \mathrm{e}^{-2 s \mathcal{V}} \varphi^{2}|q|^{2}\right) .
\end{aligned}
$$

We now have to estimate the trace of the pressure. To do this, we follow the method introduced in [12] and in [10]. We define

$$
v^{*}=s^{1 / 4} \mathrm{e}^{-s \mathcal{V}^{*}}\left(\varphi^{*}\right)^{1 / 4} v, q^{*}=s^{1 / 4} \mathrm{e}^{-s \mathcal{V}^{*}}\left(\varphi^{*}\right)^{1 / 4} q, \dot{b}^{*}=s^{1 / 4} \mathrm{e}^{-s \mathcal{V}^{*}}\left(\varphi^{*}\right)^{1 / 4} \dot{b}, \gamma^{*}=s^{1 / 4} \mathrm{e}^{-s \mathcal{V}^{*}}\left(\varphi^{*}\right)^{1 / 4} \gamma .
$$

Moreover, we impose that $b^{*}(T)=0$. We prove that $\left(v^{*}, q^{*}, \dot{b}^{*}, \gamma^{*}\right)$ is solution of a problem similar to (1.22). Next, according to the regularity result given by Proposition 2 , we know that $q^{*}$ belongs to $L^{2}\left(0, T ; H^{1}\left(\widetilde{\Omega}_{F}(t)\right)\right)$ and its norm in this space is bounded by the norm of the right-hand sides in $L^{2}$. Consequently, after several computations, we obtain that

$$
\int_{0}^{T}\left\|q^{*}(t)\right\|_{H^{1 / 2}\left(\partial \Omega \cup \partial \widetilde{\Omega}_{S}(t)\right)}^{2} \leq \widetilde{C}\left(s^{5 / 2} \int_{0}^{T} \int_{\widetilde{\Omega}_{F}(t)} \mathrm{e}^{-2 s \mathcal{v}} \varphi^{3}|v|^{2}+s^{5 / 2} \int_{0}^{T} \mathrm{e}^{-2 s \mathcal{V}^{*}}\left(\varphi^{*}\right)^{3}\left(|\dot{b}|^{2}+|\gamma|^{2}\right)\right) .
$$

Therefore, inequality (2.12) becomes, thanks to (2.15) and (2.16), for $s \geq s_{6}$ and $\lambda \geq \lambda_{6}$ where $s_{6}$ and $\lambda_{6}$ only depend on $T, \alpha, \beta_{0}$ and the norm of $\tilde{u}$ in $L^{\infty}((0, T) \times \Omega)$,

$$
\begin{gathered}
\int_{0}^{T} \int_{\tilde{\Omega}_{F}(t)} \mathrm{e}^{-2 s \mathcal{V}}\left(\frac{1}{s \varphi}\left(|\Delta v|^{2}+\left|\partial_{t} v\right|^{2}\right)+s \lambda^{2} \varphi|\nabla v|^{2}+s^{3} \lambda^{4} \varphi^{3}|v|^{2}\right)+s \lambda \int_{0}^{T} \mathrm{e}^{-2 s \mathcal{V}^{*}} \varphi^{*}\left(|\ddot{b}|^{2}+|\dot{\gamma}|^{2}\right) \\
\quad+s^{3} \lambda^{3} \int_{0}^{T} \int_{\partial \tilde{\Omega}_{S}(t)} \mathrm{e}^{-2 s \mathcal{V}} \varphi^{3}|v|^{2}+s \lambda \int_{0}^{T} \int_{\partial \tilde{\Omega}_{S}(t)} \mathrm{e}^{-2 s \mathcal{V}} \varphi|\nabla v n|^{2} \\
\leq \widetilde{C}\left(s^{2} \lambda^{2} \int_{0}^{T} \int_{\omega_{1}} \mathrm{e}^{-2 s \mathcal{V}} \varphi^{2}|q|^{2}+\int_{0}^{T} \int_{\omega_{0}} \mathrm{e}^{-2 s \mathcal{V}}\left(s^{3} \lambda^{4} \varphi^{3}|v|^{2}+s \lambda^{2} \varphi|\nabla v|^{2}\right)\right) .
\end{gathered}
$$

\subsection{Estimate on the local integral of the pressure}

We follow the arguments employed in [10]. Until now, we did not need hypotheses on the acceleration of the given fluid and structure motion (i.e. on $\partial_{t} \tilde{u}, \ddot{\tilde{a}}$ and $\dot{\tilde{r}}$ ). It will be necessary in this subsection to obtain estimates on $\partial_{t} v$. First of all, we define the time-dependent weight

$$
\hat{\mu}(t)=s \lambda \mathrm{e}^{-s \hat{v}} \hat{\varphi}
$$

Then, according to (1.28)-(1.29) and to Poincaré-Wirtinger's inequality, we have

$$
s^{2} \lambda^{2} \int_{0}^{T} \int_{\omega_{1}} \mathrm{e}^{-2 s \mathcal{V}} \varphi^{2}|q|^{2} \leq \int_{0}^{T} \int_{\omega_{1}} \hat{\mu}^{2}|q|^{2} \leq C \int_{0}^{T} \int_{\omega_{1}} \hat{\mu}^{2}|\nabla q|^{2},
$$

if we prescribe the condition on $q$ :

$$
\int_{\omega_{1}} q(t)=0
$$


From the first equation of system (1.22), we obtain

$$
\begin{aligned}
s^{2} \lambda^{2} \int_{0}^{T} \int_{\omega_{1}} \mathrm{e}^{-2 s \mathcal{V}} \varphi^{2}|\nabla q|^{2} \leq & C\left(\int_{0}^{T} \int_{\omega_{1}} \hat{\mu}^{2}|\Delta v|^{2}+\int_{0}^{T} \int_{\omega_{1}} \hat{\mu}^{2}\left|\partial_{t} v\right|^{2}\right. \\
& \left.+\|\tilde{u}\|_{L^{\infty}((0, T) \times \Omega)}^{2} \int_{0}^{T} \int_{\omega_{1}} \hat{\mu}^{2}|\nabla v|^{2}\right) .
\end{aligned}
$$

Now, we want to get estimates on the local integrals of $v$ in the right-hand side of this inequality. To begin with, we consider the term in $\Delta v$. Let us define an open set $\omega_{2}$ such that $\omega_{1} \subset \subset \omega_{2} \subset \subset \omega$. We introduce the function

$$
\hat{\mu} \chi_{0} \Delta v(T-t)
$$

where $\chi_{0}$ belongs to $\mathcal{C}_{c}^{2}\left(\omega_{2}\right)$ and $\chi_{0}=1$ in $\omega_{1}$ and we consider the problem satisfied by this function. In this stage, we can exactly use the arguments developed in [10] as if there was no structure. Indeed, the treatment is completely local, and thus the motion of the structure does not interfere. Therefore, we directly give the final estimate:

$$
\int_{0}^{T} \int_{\omega_{1}} \hat{\mu}^{2}|\Delta v|^{2} \leq C\left(\int_{0}^{T} \int_{\omega_{2}}|\dot{\hat{\mu}}|^{2}|v|^{2}+\int_{0}^{T} \int_{\omega_{2}} \hat{\mu}^{2}\left(\|\tilde{u}\|_{L^{\infty}((0, T) \times \Omega)}^{2}|\nabla v|^{2}+|v|^{2}\right)\right) .
$$

The difficult part of the proof lies in obtaining estimates on $\partial_{t} v$. Contrarily to the estimate on $\Delta v$, a local treatment can not be done. Therefore, we have to consider the global fluid-structure problem and the action of the structure on the fluid motion. Let us define a new time-dependent weight $\mu$ :

$$
\mu(t)=s^{15 / 4} \mathrm{e}^{-2 s \hat{\mathcal{V}}+s \mathcal{V}^{*}} \hat{\varphi}^{15 / 4} .
$$

We define $(\hat{v}, \hat{\pi}, \dot{\hat{c}}, \hat{\tau})=(\mu v, \mu q, \mu \dot{b}, \mu \gamma)$ and we suppose that $\hat{c}(T)=0$. We notice that $(\hat{v}, \hat{\pi}, \dot{\hat{c}}, \hat{\tau})$ is solution of the problem, for all $t \in(0, T)$

$$
\left\{\begin{array}{l}
\left(-\partial_{t} \hat{v}-(\tilde{u} \cdot \nabla) \hat{v}\right)(t, x)-\operatorname{div} \sigma(\hat{v}, \hat{\pi})(t, x)=-\dot{\mu}(t) v(t, x), \forall x \in \widetilde{\Omega}_{F}(t), \\
m \ddot{\hat{c}}(t)=-\int_{\partial \widetilde{\Omega}_{S}(t)} \sigma(\hat{v}, \hat{\pi}) n+m \dot{\mu}(t) \dot{b} \\
\dot{J} \dot{\hat{\tau}}(t)=-\int_{\partial \widetilde{\Omega}_{S}(t)}(\sigma(\hat{v}, \hat{\pi}) n) \cdot(x-\tilde{a}(t))^{\perp}+J \dot{\mu}(t) \gamma(t), \\
\operatorname{div} \hat{v}(t, x)=0, \forall x \in \widetilde{\Omega}_{F}(t), \\
\hat{v}(t, x)=0, \forall x \in \partial \Omega \\
\hat{v}(t, x)=\dot{\hat{c}}(t)+\hat{\tau}(t)(x-\tilde{a}(t))^{\perp}, \forall x \in \partial \widetilde{\Omega}_{S}(t), \\
\hat{v}(T, \cdot)=0 \text { in } \widetilde{\Omega}_{F}(T), \hat{c}(T)=0, \dot{\hat{c}}(T)=0, \hat{\tau}(T)=0 .
\end{array}\right.
$$

Now, we come back to the term we want to estimate:

$$
\int_{0}^{T} \int_{\omega_{1}} \hat{\mu}^{2}\left|\partial_{t} v\right|^{2}=\int_{0}^{T} \int_{\omega_{1}} \hat{\mu}^{2} \mu^{-2}\left|\mu \partial_{t} v\right|^{2}=\int_{0}^{T} \int_{\omega_{1}} \hat{\mu}^{2} \mu^{-2}\left|\partial_{t} \hat{v}-\dot{\mu} v\right|^{2} .
$$

By integrating by parts in time, we get

$$
\int_{0}^{T} \int_{\omega_{1}} \hat{\mu}^{2} \mu^{-2}\left|\partial_{t} \hat{v}\right|^{2}=\frac{1}{2} \int_{0}^{T} \int_{\omega_{1}} \frac{\mathrm{d}^{2}}{\mathrm{~d} t^{2}}\left(\hat{\mu}^{2} \mu^{-2}\right)|\hat{v}|^{2}-\int_{0}^{T} \int_{\omega_{1}} \hat{\mu}^{2} \mu^{-2} \partial_{t t} \hat{v} \cdot \hat{v} .
$$


Therefore, since the weight function in the integral in $|\hat{v}|^{2}$ is bounded, we obtain:

$$
\int_{0}^{T} \int_{\omega_{1}} \hat{\mu}^{2} \mu^{-2}\left|\partial_{t} \hat{v}\right|^{2} \leq C \int_{0}^{T} \int_{\omega_{1}}|\hat{v}|^{2}-\lambda^{6} \int_{0}^{T} \int_{\omega_{1}} \mu^{*} \partial_{t t} \hat{v} \cdot \hat{v}
$$

where $\mu^{*}$ is defined by

Moreover, we have

$$
\mu^{*}=\frac{1}{\lambda^{6}} \hat{\mu}^{2} \mu^{-2}=s^{-11 / 2} \lambda^{-4} \mathrm{e}^{-2 s \mathcal{V}^{*}+2 s \hat{\mathcal{V}}} \hat{\varphi}^{-11 / 2} .
$$

Consequently,

$$
-\lambda^{6} \int_{0}^{T} \int_{\omega_{1}} \mu^{*} \partial_{t t} \hat{v} \cdot \hat{v} \leq \frac{1}{2} \int_{0}^{T} \int_{\omega_{1}}\left|\mu^{*}\right|^{2}\left|\partial_{t t} \hat{v}\right|^{2}+\frac{1}{2} \lambda^{12} \int_{0}^{T} \int_{\omega_{1}}|\hat{v}|^{2} .
$$

Thus, relation (2.21) becomes

$$
\int_{0}^{T} \int_{\omega_{1}} \hat{\mu}^{2} \mu^{-2}\left|\partial_{t} \hat{v}\right|^{2} \leq \frac{1}{2} \int_{0}^{T} \int_{\omega_{1}}\left|\mu^{*}\right|^{2}\left|\partial_{t t} \hat{v}\right|^{2}+C \lambda^{12} \int_{0}^{T} \int_{\omega_{1}} \mu^{2}|v|^{2} .
$$

$$
\int_{0}^{T} \int_{\omega_{1}} \hat{\mu}^{2}\left|\partial_{t} v\right|^{2} \leq C \int_{0}^{T} \int_{\omega_{1}}\left(\lambda^{12} \mu^{2}+\dot{\mu}^{2}\right)|v|^{2}+\int_{0}^{T} \int_{\omega_{1}}\left|\mu^{*}\right|^{2}\left|\partial_{t t} \hat{v}\right|^{2}
$$

since

$$
\hat{\mu}^{2} \mu^{-2}=s^{-11 / 2} \lambda^{2} \mathrm{e}^{-2 s \mathcal{V}^{*}+2 s \hat{\mathcal{V}}} \hat{\varphi}^{-11 / 2}
$$

is bounded on $(0, T)$. We will now concentrate on the integral involving $\partial_{t t} \hat{v}$. To obtain an estimate on this term, we use Proposition 3 which is given for a direct problem but holds equivalently for an adjoint system. Considering the problem satisfied by $\left(\mu^{*} \hat{v}, \mu^{*} \hat{\pi}, \mu^{*} \dot{\hat{c}}, \mu^{*} \hat{\tau}\right)$, we have, in particular

$$
\begin{aligned}
& \left\|\mu^{*} \hat{v}\right\|_{H^{2}\left(0, T ; L^{2}\left(\omega_{1}\right)\right)^{2}} \leq\left\|\mu^{*} \hat{v}\right\|_{H^{2}\left(0, T ; L^{2}\left(\tilde{\Omega}_{F}(t)\right)\right)^{2}} \leq C\left(\left\|\mu^{*} \dot{\mu} v\right\|_{H^{1}\left(0, T ; L^{2}\left(\tilde{\Omega}_{F}(t)\right)\right)^{2}}\right. \\
& \left.+\left\|\dot{\mu}^{*} \hat{v}\right\|_{H^{1}\left(0, T ; L^{2}\left(\widetilde{\Omega}_{F}(t)\right)\right)^{2}}+\left\|\mu^{*} \dot{\mu} \dot{b}\right\|_{H^{1}(0, T)^{2}}+\left\|\dot{\mu}^{*} \dot{\hat{c}}\right\|_{H^{1}(0, T)^{2}}+\left\|\mu^{*} \dot{\mu} \gamma\right\|_{H^{1}(0, T)}+\left\|\dot{\mu}^{*} \hat{\tau}\right\|_{H^{1}(0, T)}\right),
\end{aligned}
$$

where the constant $C$ depends on $T, \alpha$, the norm of $\tilde{u}$ in $L^{\infty}\left(0, T ; L^{\infty}\left(\widetilde{\Omega}_{F}(t)\right)\right)^{2}$ and in $W^{1,4}\left(0, T ; L^{4}\left(\widetilde{\Omega}_{F}(t)\right)\right)^{2}$ and the norm of $(\tilde{a}, \tilde{r})$ in $H^{2}(0, T)^{2} \times H^{1}(0, T)$. Thus

$$
\begin{aligned}
& \left\|\mu^{*} \partial_{t t} \hat{v}\right\|_{L^{2}\left((0, T) \times \omega_{1}\right)^{2}} \leq C\left(\left\|\dot{\mu}^{*} \partial_{t} \hat{v}\right\|_{L^{2}\left((0, T) \times \omega_{1}\right)^{2}}+\left\|\ddot{\mu}^{*} \hat{v}\right\|_{L^{2}\left((0, T) \times \omega_{1}\right)^{2}}\right. \\
& +\left\|\mu^{*} \dot{\mu} v\right\|_{H^{1}\left(0, T ; L^{2}\left(\tilde{\Omega}_{F}(t)\right)\right)^{2}}+\left\|\dot{\mu}^{*} \hat{v}\right\|_{H^{1}\left(0, T ; L^{2}\left(\tilde{\Omega}_{F}(t)\right)\right)^{2}}+\left\|\mu^{*} \dot{\mu} \dot{b}\right\|_{H^{1}(0, T)^{2}}+\left\|\dot{\mu}^{*} \dot{\hat{c}}\right\|_{H^{1}(0, T)^{2}} \\
& \left.+\left\|\mu^{*} \dot{\mu} \gamma\right\|_{H^{1}(0, T)}+\left\|\dot{\mu}^{*} \hat{\tau}\right\|_{H^{1}(0, T)}\right) .
\end{aligned}
$$

Now, we come back to the variables $(v, \dot{b}, \gamma)$. We have

$$
\begin{gathered}
\left\|\mu^{*} \partial_{t t} \hat{v}\right\|_{L^{2}\left((0, T) \times \omega_{1}\right)^{2}} \leq C\left(\left\|\dot{\mu}^{*} \mu v\right\|_{H^{1}\left(0, T ; L^{2}\left(\widetilde{\Omega}_{F}(t)\right)\right)^{2}}+\left\|\ddot{\mu}^{*} \mu v\right\|_{L^{2}\left(0, T ; L^{2}\left(\widetilde{\Omega}_{F}(t)\right)\right)^{2}}\right. \\
\left.+\left\|\mu^{*} \dot{\mu} v\right\|_{H^{1}\left(0, T ; L^{2}\left(\widetilde{\Omega}_{F}(t)\right)\right)^{2}}+\left\|\mu^{*} \dot{\mu} \dot{b}\right\|_{H^{1}(0, T)^{2}}+\left\|\dot{\mu}^{*} \mu \dot{b}\right\|_{H^{1}(0, T)^{2}}+\left\|\mu^{*} \dot{\mu} \gamma\right\|_{H^{1}(0, T)}+\left\|\dot{\mu}^{*} \mu \gamma\right\|_{H^{1}(0, T)}\right) .
\end{gathered}
$$

Thus, (2.22) becomes

$$
\begin{aligned}
& \left\|\hat{\mu} \partial_{t} v\right\|_{L^{2}\left((0, T) \times \omega_{1}\right)}^{2} \leq C\left(\lambda^{12}\|\mu v\|_{L^{2}\left((0, T) \times \omega_{1}\right)}^{2}+\|\dot{\mu} v\|_{L^{2}\left((0, T) \times \omega_{1}\right)}^{2}\right. \\
& +\left\|\dot{\mu}^{*} \mu v\right\|_{H^{1}\left(0, T ; L^{2}\left(\tilde{\Omega}_{F}(t)\right)\right)^{2}}^{2}+\left\|\ddot{\mu}^{*} \mu v\right\|_{L^{2}\left(0, T ; L^{2}\left(\tilde{\Omega}_{F}(t)\right)\right)^{2}}^{2}+\left\|\mu^{*} \dot{\mu} v\right\|_{H^{1}\left(0, T ; L^{2}\left(\tilde{\Omega}_{F}(t)\right)\right)^{2}}^{2} \\
& \left.+\left\|\mu^{*} \dot{\mu} \dot{b}\right\|_{H^{1}(0, T)^{2}}^{2}+\left\|\dot{\mu}^{*} \mu \dot{b}\right\|_{H^{1}(0, T)^{2}}^{2}+\left\|\mu^{*} \dot{\mu} \gamma\right\|_{H^{1}(0, T)}^{2}+\left\|\dot{\mu}^{*} \mu \gamma\right\|_{H^{1}(0, T)}^{2}\right) .
\end{aligned}
$$


Therefore, on the one hand, we have local integrals on the velocity $v$ which we will keep in our final Carleman estimate and, on the other hand, we have global integrals in $v, \dot{b}$ and $\gamma$ and their first derivatives with respect to time which will be eliminated thanks to the estimate (2.17). Indeed, we notice that

$$
\left|\mu^{*} \dot{\mu}\right|+\left|\dot{\mu}^{*} \mu\right| \leq C s^{-3 / 4} \lambda^{-4} \mathrm{e}^{-s \mathcal{V}^{*}} \hat{\varphi}^{-1 / 2},\left|\ddot{\mu}^{*} \mu\right|+\left|\dot{\mu}^{*} \dot{\mu}\right|+\left|\mu^{*} \ddot{\mu}\right| \leq C s^{1 / 4} \lambda^{-4} \mathrm{e}^{-s \mathcal{V}^{*}} \hat{\varphi}^{3 / 4} .
$$

Therefore, according to definition (1.10), we get

$$
\begin{gathered}
\left.\int_{0}^{T} \int_{\omega_{1}}\left|\hat{\mu}^{2}\right| \partial_{t} v\right|^{2} \leq C\left(\int_{0}^{T} \int_{\omega_{1}}\left(\lambda^{12} \mu^{2}+\dot{\mu}^{2}\right)|v|^{2}+s^{1 / 2} \lambda^{-8} \int_{0}^{T} \int_{\widetilde{\Omega}_{F}(t)} \mathrm{e}^{-2 s \mathcal{V}^{*}} \hat{\varphi}^{3 / 2}|v|^{2}\right. \\
+s^{1 / 2} \lambda^{-8} \int_{0}^{T} \mathrm{e}^{-2 s \mathcal{V}^{*}} \hat{\varphi}^{3 / 2}\left(|\dot{b}|^{2}+|\gamma|^{2}\right)+s^{-3 / 2} \lambda^{-8} \int_{0}^{T} \int_{\widetilde{\Omega}_{F}(t)} \mathrm{e}^{-2 s \mathcal{V}^{*}} \hat{\varphi}^{-1}\left(\left|\partial_{t} v\right|^{2}+|\nabla v|^{2}\right) \\
\left.+s^{-3 / 2} \lambda^{-8} \int_{0}^{T} \mathrm{e}^{-2 s \mathcal{V}^{*}} \hat{\varphi}^{-1}\left(|\ddot{b}|^{2}+|\dot{\gamma}|^{2}\right)\right)
\end{gathered}
$$

where the constant $C$ depends on $T$, $\alpha$, the norm of $\tilde{u}$ in $L^{\infty}\left(0, T ; L^{\infty}\left(\widetilde{\Omega}_{F}(t)\right)\right)^{2}$ and in $W^{1,4}\left(0, T ; L^{4}\left(\widetilde{\Omega}_{F}(t)\right)\right)^{2}$ and the norm of $(\tilde{a}, \tilde{r})$ in $H^{2}(0, T)^{2} \times H^{1}(0, T)$. It remains to reassemble all these terms to obtain an estimate on the local integral on the pressure. Thanks to (2.19) and the last inequality, (2.18) becomes

$$
\begin{aligned}
& s^{2} \lambda^{2} \int_{0}^{T} \int_{\omega_{1}} \mathrm{e}^{-2 s \mathcal{V}} \varphi^{2}|q|^{2} \leq C\left(\int_{0}^{T} \int_{\omega_{2}} \hat{\mu}^{2}|\nabla v|^{2}+\int_{0}^{T} \int_{\omega_{2}}\left(\lambda^{12} \mu^{2}+|\dot{\mu}|^{2}\right)|v|^{2}\right. \\
& +s^{1 / 2} \lambda^{-8} \int_{0}^{T} \int_{\widetilde{\Omega}_{F}(t)} \mathrm{e}^{-2 s \mathcal{V}^{*}} \hat{\varphi}^{3 / 2}|v|^{2}+s^{1 / 2} \lambda^{-8} \int_{0}^{T} \mathrm{e}^{-2 s \mathcal{V}^{*}} \hat{\varphi}^{3 / 2}\left(|\dot{b}|^{2}+|\gamma|^{2}\right) \\
& \left.+s^{-3 / 2} \lambda^{-8} \int_{0}^{T} \int_{\widetilde{\Omega}_{F}(t)} \mathrm{e}^{-2 s \mathcal{V}^{*}} \hat{\varphi}^{-1}\left(\left|\partial_{t} v\right|^{2}+|\nabla v|^{2}\right)+s^{-3 / 2} \lambda^{-8} \int_{0}^{T} \mathrm{e}^{-2 s \mathcal{V}^{*}} \hat{\varphi}^{-1}\left(|\ddot{b}|^{2}+|\dot{\gamma}|^{2}\right)\right) .
\end{aligned}
$$

We see that terms in $v, b$ and $\gamma$ can be eliminated using the left-hand side of (2.17). Finally inequality (2.17) becomes

$$
\begin{gathered}
\int_{0}^{T} \int_{\widetilde{\Omega}_{F}(t)} \mathrm{e}^{-2 s \mathcal{V}}\left(\frac{1}{s \varphi}\left(|\Delta v|^{2}+\left|\partial_{t} v\right|^{2}\right)+s \lambda^{2} \varphi|\nabla v|^{2}+s^{3} \lambda^{4} \varphi^{3}|v|^{2}\right)+s \lambda \int_{0}^{T} \mathrm{e}^{-2 s \mathcal{V}^{*}} \varphi^{*}\left(|\ddot{b}|^{2}+|\dot{\gamma}|^{2}\right) \\
+s^{3} \lambda^{3} \int_{0}^{T} \int_{\partial \widetilde{\Omega}_{S}(t)} \mathrm{e}^{-2 s \mathcal{V}} \varphi^{3}|v|^{2}+s \lambda \int_{0}^{T} \int_{\partial \widetilde{\Omega}_{S}(t)} \mathrm{e}^{-2 s \mathcal{V}} \varphi|\nabla v n|^{2} \\
\leq \widetilde{C}\left(\int_{0}^{T} \int_{\omega_{2}} \hat{\mu}^{2}|\nabla v|^{2}+\int_{0}^{T} \int_{\omega_{2}}\left(\lambda^{12} \mu^{2}+|\dot{\mu}|^{2}\right)|v|^{2}\right),
\end{gathered}
$$

for $s \geq s_{7}$ and $\lambda \geq \lambda_{7}$ where $s_{7}$ and $\lambda_{7}$ depend on $T, \alpha, \beta_{0}$, the norms of $\tilde{a}$ in $H^{2}(0, T)^{2}, \tilde{r}$ in $H^{1}(0, T)$ and $\tilde{u}$ in $L^{\infty}((0, T) \times \Omega)^{2} \cap W^{1,4}\left(0, T ; L^{4}\left(\widetilde{\Omega}_{F}(t)\right)\right)^{2}$. To conclude the proof, we notice that it is not necessary to have a control both on $v$ and on $\nabla v$. Indeed, if we consider a function $\chi_{1}$ belonging to $\mathcal{C}_{c}^{2}(\omega)$ such that

$$
0 \leq \chi_{1} \leq 1, \chi_{1}=1 \text { in } \omega_{2}
$$


we notice that

$$
\begin{aligned}
\int_{0}^{T} \int_{\omega_{2}} \hat{\mu}^{2}|\nabla v|^{2} & \leq \int_{0}^{T} \int_{\omega} \chi_{1} \hat{\mu}^{2}|\nabla v|^{2}=-\int_{0}^{T} \int_{\omega} \operatorname{div}\left(\chi_{1} \hat{\mu}^{2} \nabla v\right) \cdot v \\
& \leq \frac{1}{2} \int_{0}^{T} \int_{\omega} \hat{\mu}^{2} \Delta \chi_{1}|v|^{2}-\int_{0}^{T} \int_{\omega} \hat{\mu}^{2} \chi_{1} \Delta v \cdot v \\
& \leq C\left(\int_{0}^{T} \int_{\omega} \hat{\mu}^{2}|v|^{2}+\frac{\epsilon}{s \lambda} \int_{0}^{T} \int_{\omega} \mathrm{e}^{-2 s \mathcal{V}^{*}} \frac{1}{\hat{\varphi}}|\Delta v|^{2}+\frac{s \lambda}{\epsilon} \int_{0}^{T} \int_{\omega} \mathrm{e}^{2 s \mathcal{V}^{*}} \hat{\varphi} \hat{\mu}^{4}|v|^{2}\right) \\
& \leq C\left(\frac{\epsilon}{s \lambda} \int_{0}^{T} \int_{\tilde{\Omega}_{F}(t)} \mathrm{e}^{-2 s \mathcal{V}} \frac{1}{\varphi}|\Delta v|^{2}+\frac{s^{5} \lambda^{5}}{\epsilon} \int_{0}^{T} \int_{\omega} \mathrm{e}^{2 s \mathcal{V}^{*}-4 s \hat{\mathcal{V}}} \hat{\varphi}^{5}|v|^{2}\right),
\end{aligned}
$$

for $\epsilon>0$ sufficiently small. Thus, (2.23) becomes, for $s \geq s_{8}, \lambda \geq \lambda_{8}$,

$$
\begin{gathered}
\int_{0}^{T} \int_{\tilde{\Omega}_{F}(t)} \mathrm{e}^{-2 s \mathcal{V}}\left(\frac{1}{s \varphi}\left(|\Delta v|^{2}+\left|\partial_{t} v\right|^{2}\right)+s \lambda^{2} \varphi|\nabla v|^{2}+s^{3} \lambda^{4} \varphi^{3}|v|^{2}\right)+s \lambda \int_{0}^{T} \mathrm{e}^{-2 s \mathcal{V}^{*}} \varphi^{*}\left(|\ddot{b}|^{2}+|\dot{\gamma}|^{2}\right) \\
+s^{3} \lambda^{3} \int_{0}^{T} \int_{\partial \tilde{\Omega}_{S}(t)} \mathrm{e}^{-2 s \mathcal{V}} \varphi^{3}|v|^{2}+s \lambda \int_{0}^{T} \int_{\partial \tilde{\Omega}_{S}(t)} \mathrm{e}^{-2 s \mathcal{V}} \varphi|\nabla v n|^{2} \\
\leq C\left(s^{19 / 2} \lambda^{12} \int_{0}^{T} \int_{\omega} \mathrm{e}^{2 s \mathcal{V}^{*}-4 s \hat{\mathcal{V}}} \hat{\varphi}^{10}|v|^{2}\right) .
\end{gathered}
$$

The constants $C, s_{8}, \lambda_{8}$ depend on $T, \alpha, \beta_{0}$ and the norms of $\tilde{a}$ in $H^{2}(0, T)^{2}, \tilde{r}$ in $H^{1}(0, T)$ and $\tilde{u}$ in $L^{\infty}((0, T) \times$ $\Omega)^{2} \cap W^{1,4}\left(0, T ; L^{4}\left(\widetilde{\Omega}_{F}(t)\right)\right)^{2}$. Thus, we obtain inequality (1.30) with a constant $C$ depending only on $T, \alpha$ and $\beta_{0}$.

\section{Null CONTROllability of the Linear System}

In this section, we will prove Theorem 2. Under the hypotheses given in this theorem, we will prove the existence of a control $f$ such that the solution of (1.21) satisfies

$$
u(T, \cdot)=0 \text { in } \widetilde{\Omega}_{F}(t), a(T)=0, \dot{a}(T)=0, \theta(T)=0, r(T)=0 .
$$

\subsection{An observability inequality}

To begin with, we will deduce from Theorem 3 an observability inequality for the adjoint problem (1.22) associated to the linearized problem.

Proposition 4. We consider $s \geq \hat{s}$ and $\lambda \geq \hat{\lambda}$ such that Carleman inequality (1.30) holds. For any $\tilde{a} \in$ $H^{2}(0, T)^{2}, \tilde{r} \in H^{1}(0, T)$ satisfying (1.17) for some $\alpha>0$ and $\tilde{u}$ satisfying conditions (1.18) to (1.20) such that

$$
\|\tilde{a}\|_{H^{2}(0, T)^{2}}+\|\tilde{r}\|_{H^{1}(0, T)}+\|\tilde{u}\|_{L^{\infty}\left(0, T ; L^{\infty}\left(\tilde{\Omega}_{F}(t)\right)\right)^{2}}+\|\tilde{u}\|_{W^{1,4}\left(0, T ; L^{4}\left(\tilde{\Omega}_{F}(t)\right)\right)^{2}} \leq R,
$$

there exists a constant $C$ depending on $T, \alpha, R, s$ and $\lambda$ such that every solution of (1.22) satisfies

$$
\begin{aligned}
& \int_{\Omega_{F}(0)}|v(0)|^{2}+|\dot{b}(0)|^{2}+|\gamma(0)|^{2}+\int_{0}^{T} \int_{\tilde{\Omega}_{F}(t)} \mathrm{e}^{-2 s \mathcal{V}}\left(\frac{1}{\varphi}\left(|\Delta v|^{2}+\left|\partial_{t} v\right|^{2}\right)+\varphi|\nabla v|^{2}+\varphi^{3}|v|^{2}\right) \\
& +\int_{0}^{T} \int_{\partial \tilde{\Omega}_{S}(t)} \mathrm{e}^{-2 s \mathcal{V}} \varphi^{3}|v|^{2} \leq C \int_{0}^{T} \int_{\omega} \mathrm{e}^{2 s \mathcal{V}^{*}-4 s \hat{\mathcal{V}}} \hat{\varphi}^{10}|v|^{2} .
\end{aligned}
$$


Proof of Proposition 4. Let us define a function $\bar{\eta} \in \mathcal{C}^{1}(0, T)$ such that

$$
\bar{\eta}=1 \text { on }[0, T / 2], \bar{\eta}=0 \text { on }[3 T / 4, T]
$$

Next, we consider $(\bar{v}, \bar{q}, \dot{\bar{b}}, \bar{\gamma})=(\bar{\eta} v, \bar{\eta} q, \bar{\eta} \dot{b}, \bar{\eta} \gamma)$ such that $\bar{b}(T)=0$ where $(v, q, b, s)$ is solution of (1.22). It satisfies the following system: for all $t \in(0, T)$,

$$
\left\{\begin{array}{l}
-\partial_{t} \bar{v}-(\tilde{u} \cdot \nabla) \bar{v}-\operatorname{div} \sigma(\bar{v}, \bar{q})=-\dot{\bar{\eta}} v \text { in } \widetilde{\Omega}_{F}(t), \\
m \ddot{\bar{b}}=-\int_{\partial \widetilde{\Omega}_{S}(t)} \sigma(\bar{v}, \bar{q}) n+m \dot{\bar{\eta}} \dot{b}, \\
J \dot{\bar{\gamma}}=-\int_{\partial \widetilde{\Omega}_{S}(t)}(\sigma(\bar{v}, \bar{q}) n) \cdot(x-\tilde{a})^{\perp}+J \dot{\bar{\eta}} \gamma, \\
\operatorname{div} \bar{v}=0 \text { in } \widetilde{\Omega}_{F}(t), \\
\bar{v}=0 \text { on } \partial \Omega, \\
\bar{v}=\dot{\bar{b}}+\bar{\gamma}(x-\tilde{a})^{\perp} \text { on } \partial \widetilde{\Omega}_{S}(t), \\
\bar{v}(T, \cdot)=0 \text { in } \widetilde{\Omega}_{F}(T), \dot{\bar{b}}(T)=0, \bar{b}(T)=0, \bar{\gamma}(T)=0 .
\end{array}\right.
$$

Thanks to Proposition 2 applied to this adjoint problem, we have

$$
\begin{aligned}
\|\bar{v}\|_{\mathcal{U}\left(0, T ; \widetilde{\Omega}_{F}(t)\right)^{2}}+\|\dot{\bar{b}}\|_{H^{1}(0, T)^{2}}+\|\bar{\gamma}\|_{H^{1}(0, T) \leq} & C\left(\|\dot{\bar{\eta}} v\|_{L^{2}\left(0, T ; L^{2}\left(\widetilde{\Omega}_{F}(t)\right)\right)^{2}}+\|\dot{\bar{\eta}} \dot{b}\|_{L^{2}(0, T)^{2}}\right. \\
& \left.+\|\dot{\bar{\eta}} \gamma\|_{L^{2}(0, T)}\right) .
\end{aligned}
$$

This implies that

$$
\begin{aligned}
\|v\|_{\mathcal{U}\left(0, T / 2 ; \widetilde{\Omega}_{F}(t)\right)^{2}}+\|\dot{b}\|_{H^{1}(0, T / 2)^{2}}+\|\gamma\|_{H^{1}(0, T / 2)} \leq & C\left(\|v\|_{L^{2}\left(T / 2,3 T / 4 ; L^{2}\left(\widetilde{\Omega}_{F}(t)\right)\right)^{2}}\right. \\
& \left.+\|\dot{b}\|_{L^{2}(T / 2,3 T / 4)^{2}}+\|\gamma\|_{L^{2}(T / 2,3 T / 4)}\right) .
\end{aligned}
$$

Therefore, since the weight functions are bounded in $[T / 2,3 T / 4]$, we have

$$
\begin{aligned}
\int_{\Omega_{F}(0)}|v(0)|^{2} & +|\dot{b}(0)|^{2}+|\gamma(0)|^{2} \leq C\left(\int_{T / 2}^{3 T / 4} \int_{\widetilde{\Omega}_{F}(t)} \mathrm{e}^{-2 s \mathcal{V}} \varphi^{3}|v|^{2}\right. \\
& \left.+\int_{T / 2}^{3 T / 4} \mathrm{e}^{-2 s \mathcal{V}^{*}}\left(\varphi^{*}\right)^{3}\left(|\dot{b}|^{2}+|\gamma|^{2}\right)\right)
\end{aligned}
$$

Thus, the Carleman inequality (1.30) allows to conclude the proof of Proposition 4.

\subsection{Control of the displacement}

Arguing as in [6], we can show that the conditions on the displacement $a(T)=0$ and $\theta(T)=0$ required on system (1.21) are equivalent to two linear constraints on the control $f$. Indeed, if we define $\left(v_{1}, q_{1}, b_{1}, \gamma_{1}\right)$ and 
$\left(v_{2}, q_{2}, b_{2}, \gamma_{2}\right)$ as the solutions of the following problems

$$
\left\{\begin{array}{l}
\left(-\partial_{t} v_{1}-(\tilde{u} \cdot \nabla) v_{1}\right)(t, x)-\operatorname{div} \sigma\left(v_{1}, q_{1}\right)(t, x)=0, \forall x \in \widetilde{\Omega}_{F}(t), \\
m\left(\ddot{b}_{1}(t)+1\right)=-\int_{\partial \tilde{\Omega}_{S}(t)} \sigma\left(v_{1}, q_{1}\right) n \\
J \dot{\gamma}_{1}(t)=-\int_{\partial \tilde{\Omega}_{S}(t)}\left(\sigma\left(v_{1}, q_{1}\right) n\right) \cdot(x-\tilde{a}(t))^{\perp}, \\
\operatorname{div} v_{1}(t, x)=0, \forall x \in \widetilde{\Omega}_{F}(t), \\
v_{1}(t, x)=0, \forall x \in \partial \Omega \\
v_{1}(t, x)=\dot{b}_{1}(t)+\gamma_{1}(t)(x-\tilde{a}(t))^{\perp}, \forall x \in \partial \widetilde{\Omega}_{S}(t), \\
v_{1}(T, \cdot)=0 \text { in } \widetilde{\Omega}_{F}(T), b_{1}(T)=0, \dot{b}_{1}(T)=0, \gamma_{1}(T)=0
\end{array}\right.
$$

and

$$
\left\{\begin{array}{l}
\left(-\partial_{t} v_{2}-(\tilde{u} \cdot \nabla) v_{2}\right)(t, x)-\operatorname{div} \sigma\left(v_{2}, q_{2}\right)(t, x)=0, \forall x \in \widetilde{\Omega}_{F}(t), \\
m \ddot{b}_{2}(t)=-\int_{\partial \tilde{\Omega}_{S}(t)} \sigma\left(v_{2}, q_{2}\right) n \\
J\left(\dot{\gamma}_{2}(t)+1\right)=-\int_{\partial \tilde{\Omega}_{S}(t)}\left(\sigma\left(v_{2}, q_{2}\right) n\right) \cdot(x-\tilde{a}(t))^{\perp}, \\
\operatorname{div} v_{2}(t, x)=0, \forall x \in \widetilde{\Omega}_{F}(t) \\
v_{2}(t, x)=0, \forall x \in \partial \Omega \\
v_{2}(t, x)=\dot{b}_{2}(t)+\gamma_{2}(t)(x-\tilde{a}(t))^{\perp}, \forall x \in \partial \widetilde{\Omega}_{S}(t), \\
v_{2}(T, \cdot)=0 \text { in } \widetilde{\Omega}_{F}(T), b_{2}(T)=0, \dot{b}_{2}(T)=0, \gamma_{2}(T)=0
\end{array}\right.
$$

a classical computation leads to

$$
\begin{array}{r}
\int_{0}^{T} \int_{\omega} f \cdot v_{1}=-m \dot{b}_{1}(0) \cdot a_{1}-J \gamma_{1}(0) r_{0}-\int_{\Omega_{F}(0)} v_{1}(0) \cdot u_{0}+m a(T)-m a_{0}, \\
\int_{0}^{T} \int_{\omega} f \cdot v_{2}=-m \dot{b}_{2}(0) \cdot a_{1}-J \gamma_{2}(0) r_{0}-\int_{\Omega_{F}(0)} v_{2}(0) \cdot u_{0}+J \theta(T)-J \theta_{0} .
\end{array}
$$

Consequently, $a(T)=0$ and $\theta(T)=0$ if and only if

$$
\int_{0}^{T} \int_{\omega} f \cdot v_{1}=m_{1}\left(a_{0}, a_{1}, r_{0}, u_{0}\right), \int_{0}^{T} \int_{\omega} f \cdot v_{2}=m_{2}\left(a_{1}, \theta_{0}, r_{0}, u_{0}\right),
$$

where

$$
\begin{aligned}
& m_{1}\left(a_{0}, a_{1}, r_{0}, u_{0}\right)=-m \dot{b}_{1}(0) \cdot a_{1}-J \gamma_{1}(0) r_{0}-\int_{\Omega_{F}(0)} v_{1}(0) \cdot u_{0}-m a_{0}, \\
& m_{2}\left(a_{1}, \theta_{0}, r_{0}, u_{0}\right)=-m \dot{b}_{2}(0) \cdot a_{1}-J \gamma_{2}(0) r_{0}-\int_{\Omega_{F}(0)} v_{2}(0) \cdot u_{0}-J \theta_{0} .
\end{aligned}
$$


We have to check that, for each initial condition $\left(a_{0}, a_{1}, \theta_{0}, r_{0}, u_{0}\right)$ the set of functions $f$ in $L^{2}((0, T) \times \omega)$ satisfying (3.5) is non empty. This will hold if $v_{1}$ and $v_{2}$ are non identically equal to 0 on $(0, T) \times \omega$. To prove this, we need the following unique continuation property on the fluid equations:

Lemma 6. Let $(\tilde{a}, \tilde{\theta}) \in \mathcal{C}(0, T)^{2} \times \mathcal{C}(0, T)$ be given. We define $\widetilde{\Omega}_{S}(t)$ by (1.15) and we suppose that (1.17) is satisfied. We consider the following system

$$
\left\{\begin{array}{l}
\left(-\partial_{t} v-(\tilde{u} \cdot \nabla) v\right)(t, x)-\operatorname{div} \sigma(v, q)(t, x)=0, \forall x \in \widetilde{\Omega}_{F}(t), \forall t \in(0, T), \\
\operatorname{div} v(t, x)=0, \forall x \in \widetilde{\Omega}_{F}(t), \forall t \in(0, T)
\end{array}\right.
$$

where $\widetilde{\Omega}_{F}(t)=\Omega \backslash \overline{\widetilde{\Omega}_{S}(t)}$ and $\tilde{u}$ belongs to $L^{\infty}\left(0, T ; L^{\infty}\left(\widetilde{\Omega}_{F}(t)\right)\right)^{2}$. If

$$
v(t, x)=0, \forall x \in \omega, \forall t \in(0, T),
$$

then

where $q_{0}$ is a constant.

Proof of Lemma 6 . Let $t_{0} \in(0, T)$ be fixed. We consider a given $x_{0} \in \widetilde{\Omega}_{F}\left(t_{0}\right)$. Then, there exists $0<t_{1}<t_{0}<$ $t_{2}<T$ and an open and connected set $\Omega_{1}$ such that $\left(t_{0}, x_{0}\right) \in\left(t_{1}, t_{2}\right) \times \Omega_{1}$ and

$$
\omega \subset \Omega_{1} \subset \widetilde{\Omega}_{F}(t), \forall t \in\left(t_{1}, t_{2}\right) .
$$

This comes from the regularity of $\tilde{a}$ and $\tilde{\theta}$ and from the two last conditions of (1.17). Thus, system (3.6) is satisfied in $\left(t_{1}, t_{2}\right) \times \Omega_{1}$ and we can apply the unique continuation property obtained in [7]. We deduce that $v=0$ in $\left(t_{1}, t_{2}\right) \times \Omega_{1}$ and in particular $v\left(t_{0}, x_{0}\right)=0$. This proves our result.

Thus, if we suppose that, for instance $v_{1}=0$ on $(0, T) \times \omega$, this lemma implies that

$$
v_{1}(t, x)=0, q_{1}(t, x)=q_{0}^{1}, \forall x \in \widetilde{\Omega}_{F}(t), \forall t \in(0, T),
$$

where $q_{0}^{1}$ is a constant and thus, by continuity of the velocities at the interface, $\dot{b}_{1}=0$ and $\gamma_{1}=0$ on $(0, T)$. But according to the equation satisfied by $b_{1}$, this is impossible. Thus $v_{1}$ and, with the same arguments, $v_{2}$ are non identically null on $\omega$.

To obtain a control satisfying the constraints (3.5), we follow the method presented in [15] and used in [6]. We will prove an improved observability inequality. We define the weight $\Theta$ by

$$
\Theta=\mathrm{e}^{4 s \hat{\mathcal{V}}-2 s \mathcal{V}^{*}} \hat{\varphi}^{-10} \text {. }
$$

This weight corresponds to the inverse of the weight function in the right-hand side of inequality (3.2). We denote by $P$ the orthogonal projection operator from $L^{2}((0, T) \times \omega)$ into $\operatorname{span}\left(v_{1}, v_{2}\right)$ where the measure of $L^{2}((0, T) \times \omega)$ is the weighted measure $\Theta^{-1} \mathrm{~d} x \mathrm{~d} t$. Thus, we have

$$
\int_{0}^{T} \int_{\omega} \Theta^{-1}(v-P(v)) \cdot v_{1}=\int_{0}^{T} \int_{\omega} \Theta^{-1}(v-P(v)) \cdot v_{2}=0, \forall v \in L^{2}((0, T) \times \omega) .
$$

We also introduce $P_{1}$ and $P_{2}$ the linear operators from $L^{2}((0, T) \times \omega)$ into $\mathbb{R}$ such that

$$
P(v)=P_{1}(v) v_{1}+P_{2}(v) v_{2}, \forall v \in L^{2}((0, T) \times \omega) .
$$


Proposition 5. Under the assumptions of Proposition 4, every solution of (1.22) satisfies

$$
\begin{aligned}
& \int_{\Omega_{F}(0)}|v(0)|^{2}+|\dot{b}(0)|^{2}+|\gamma(0)|^{2}+\int_{0}^{T} \int_{\widetilde{\Omega}_{F}(t)} \mathrm{e}^{-2 s \mathcal{V}}\left(\frac{1}{\varphi}\left(|\Delta v|^{2}+\left|\partial_{t} v\right|^{2}\right)+\varphi|\nabla v|^{2}+\varphi^{3}|v|^{2}\right) \\
& +\int_{0}^{T} \int_{\partial \widetilde{\Omega}_{S}(t)} \mathrm{e}^{-2 s \mathcal{V}} \varphi^{3}|v|^{2}+\left|P_{1}(v)\right|^{2}+\left|P_{2}(v)\right|^{2} \leq C \int_{0}^{T} \int_{\omega} \mathrm{e}^{2 s \mathcal{V}^{*}-4 s \hat{\mathcal{V}}} \hat{\varphi}^{10}|v-P(v)|^{2} .
\end{aligned}
$$

Proof of Proposition 5. To prove this inequality, we will argue by contradiction. Assume that this inequality does not hold and let us define, for each $n \in \mathbb{N},\left(v_{n}, q_{n}, b_{n}, \gamma_{n}\right)$ a solution of $(1.22)$ such that

$$
\begin{aligned}
1 & =\int_{\Omega_{F}(0)}\left|v_{n}(0)\right|^{2}+\left|\dot{b}_{n}(0)\right|^{2}+\left|\gamma_{n}(0)\right|^{2}+\int_{0}^{T} \int_{\widetilde{\Omega}_{F}(t)} \mathrm{e}^{-2 s \mathcal{V}}\left(\frac{1}{\varphi}\left(\left|\Delta v_{n}\right|^{2}+\left|\partial_{t} v_{n}\right|^{2}\right)+\varphi\left|\nabla v_{n}\right|^{2}\right) \\
+ & \int_{0}^{T} \int_{\widetilde{\Omega}_{F}(t)} \mathrm{e}^{-2 s \mathcal{V}} \varphi^{3}\left|v_{n}\right|^{2}+\int_{0}^{T} \int_{\partial \widetilde{\Omega}_{S}(t)} \mathrm{e}^{-2 s \mathcal{V}} \varphi^{3}\left|v_{n}\right|^{2}+\left|P_{1}\left(v_{n}\right)\right|^{2}+\left|P_{2}\left(v_{n}\right)\right|^{2} \\
& >n \int_{0}^{T} \int_{\omega} \mathrm{e}^{2 s \mathcal{V}^{*}-4 s \hat{\mathcal{V}}^{10}} \hat{\varphi}^{10}\left|v_{n}-P\left(v_{n}\right)\right|^{2} .
\end{aligned}
$$

This implies that $\left(P_{1}\left(v_{n}\right)\right)$ and $\left(P_{2}\left(v_{n}\right)\right)$ are bounded in $\mathbb{R}$ and converge, up to a subsequence, respectively to $\beta_{1}$ and $\beta_{2}$. We also deduce from (3.9) that, for all fixed $\epsilon>0$, the sequence $\left(v_{n}\right)$ is bounded in $L^{2}(\epsilon, T-\epsilon$; $\left.L^{2}\left(\widetilde{\Omega}_{F}(t)\right)\right)^{2}$ and the sequence $\left(v_{n}-P\left(v_{n}\right)\right)$ strongly converges to 0 in $L^{2}((\epsilon, T-\epsilon) \times \omega)^{2}$. Therefore, up to a subsequence, $\left(v_{n}\right)$ weakly converges to $v$ in $L^{2}\left(\epsilon, T-\epsilon ; L^{2}\left(\widetilde{\Omega}_{F}(t)\right)\right)^{2}$ and

$$
v=\beta_{1} v_{1}+\beta_{2} v_{2} \text { in }(0, T) \times \omega .
$$

Moreover, according to (3.9) and to the first equation of system (1.22), we have, up to a subsequence,

$$
v_{n} \rightarrow v \text { in } L^{2}\left(\epsilon, T-\epsilon ; H^{1}\left(\widetilde{\Omega}_{F}(t)\right)\right)^{2}, q_{n} \rightarrow q \text { in } L^{2}\left(\epsilon, T-\epsilon ; L^{2}\left(\widetilde{\Omega}_{F}(t)\right)\right)^{2} .
$$

(3.9) also implies that

$$
\int_{\epsilon}^{T-\epsilon} \int_{\partial \widetilde{\Omega}_{S}(t)}\left|v_{n}\right|^{2}
$$

is bounded, and thus, thanks to the last hypothesis of (1.9), we have, up to a subsequence,

$$
b_{n} \rightarrow b \text { in } H^{1}(\epsilon, T-\epsilon)^{2}, \gamma_{n} \rightarrow \gamma \text { in } L^{2}(\epsilon, T-\epsilon)
$$

We notice that $(v, q, b, \gamma)$ satisfies, for all $t \in(0, T)$

$$
\left\{\begin{array}{l}
\left(-\partial_{t} v-(\tilde{u} \cdot \nabla) v\right)(t, x)-\operatorname{div} \sigma(v, q)(t, x)=0, \forall x \in \widetilde{\Omega}_{F}(t), \\
m \ddot{b}(t)=-\int_{\partial \widetilde{\Omega}_{S}(t)} \sigma(v, q) n \\
J \dot{\gamma}(t)=-\int_{\partial \widetilde{\Omega}_{S}(t)}(\sigma(v, q) n) \cdot(x-\tilde{a}(t))^{\perp} \\
\operatorname{div} v(t, x)=0, \forall x \in \widetilde{\Omega}_{F}(t) \\
v(t, x)=0, \forall x \in \partial \Omega \\
v(t, x)=\dot{b}(t)+\gamma(t)(x-\tilde{a}(t))^{\perp}, \forall x \in \partial \widetilde{\Omega}_{S}(t)
\end{array}\right.
$$


Next, we notice that, if we consider the function $v-\left(\beta_{1} v_{1}+\beta_{2} v_{2}\right)$, we can apply Lemma 6 and deduce that

$$
v(t, x)=\beta_{1} v_{1}(t, x)+\beta_{2} v_{2}(t, x), \forall x \in \widetilde{\Omega}_{F}(t), \forall t \in(0, T)
$$

This implies that

$$
\dot{b}(t)=\beta_{1} \dot{b}_{1}(t)+\beta_{2} \dot{b}_{2}(t), \gamma(t)=\beta_{1} \gamma_{1}(t)+\beta_{2} \gamma_{2}(t), \forall t \in(0, T)
$$

According to the equations satisfied by $b, b_{1}$ and $b_{2}$ on one hand, and $\gamma, \gamma_{1}$ and $\gamma_{2}$ on the other hand, this is only possible for $\beta_{1}=0, \beta_{2}=0$ and thus $v=0$. Moreover, since $v_{n}=\left(v_{n}-P\left(v_{n}\right)\right)+P\left(v_{n}\right)$, $\left(v_{n}\right)$ strongly converges to 0 in $L^{2}((0, T) \times \omega)$ and, in particular,

$$
\int_{0}^{T} \int_{\omega} \mathrm{e}^{2 s \mathcal{V}^{*}-4 s \hat{\mathcal{V}}} \hat{\varphi}^{10}\left|v_{n}\right|^{2} \rightarrow 0
$$

Thus, according to $(3.2)$,

$$
\begin{aligned}
& \int_{\Omega_{F}(0)}\left|v_{n}(0)\right|^{2}+\left|\dot{b}_{n}(0)\right|^{2}+\left|\gamma_{n}(0)\right|^{2}+\int_{0}^{T} \int_{\widetilde{\Omega}_{F}(t)} \mathrm{e}^{-2 s \mathcal{V}}\left(\frac{1}{\varphi}\left(\left|\Delta v_{n}\right|^{2}+\left|\partial_{t} v_{n}\right|^{2}\right)+\varphi\left|\nabla v_{n}\right|^{2}\right) \\
& +\int_{0}^{T} \int_{\widetilde{\Omega}_{F}(t)} \mathrm{e}^{-2 s \mathcal{V}} \varphi^{3}\left|v_{n}\right|^{2}+\int_{0}^{T} \int_{\partial \widetilde{\Omega}_{S}(t)} \mathrm{e}^{-2 s \mathcal{V}} \varphi^{3}\left|v_{n}\right|^{2} \rightarrow 0 .
\end{aligned}
$$

At last, since $P_{1}$ and $P_{2}$ are continuous from $L^{2}((0, T) \times \omega)$ to $\mathbb{R}$ (this can be shown by expressing $P_{1}(v)$ and $P_{2}(v)$ in terms of $v, v_{1}$ and $\left.v_{2}\right)$, we also have

$$
\left|P_{1}\left(v_{n}\right)\right|^{2}+\left|P_{2}\left(v_{n}\right)\right|^{2} \rightarrow 0
$$

These two properties are in contradiction with hypothesis (3.9). Therefore our proposition holds.

\subsection{Null controllability result on the linear system}

We are now able to prove Theorem 2. Adapting the method used in [1], we introduce an extremal problem. For any fixed initial condition $\left(a_{0}, a_{1}, \theta_{0}, r_{0}, u_{0}\right)$ and any $\epsilon>0$, we consider the functional

$$
J_{\epsilon}(f, u, p, a, r)=\frac{1}{2 \epsilon}\left(\int_{\widetilde{\Omega}_{F}(T)}|u(T)|^{2} \mathrm{~d} x+|\dot{a}(T)|^{2}+|r(T)|^{2}\right)+\frac{1}{2} \int_{0}^{T} \int_{\omega} \Theta|f|^{2},
$$

where $(u, p, a, r)$ is the solution of (1.21) associated to $f$ and we want to minimize this functional with respect to $f$ in $L^{2}((0, T) \times \omega)^{2}$ such that $(3.5)$ holds. The set of functions satisfying these constraints is non empty thanks to Lemma 6 . For each $\epsilon>0, J_{\epsilon}$ is continuous and strictly convex. Moreover, arguing as in [8], we can prove that it is also coercive. Thus, this minimization problem admits a unique solution $\left(f_{\epsilon}, u_{\epsilon}, p_{\epsilon}, a_{\epsilon}, r_{\epsilon}\right)$. We will apply Lagrange's principle to this problem. We formally explain how we apply it. First of all, we can compute the derivative of $J_{\epsilon}$ at a point $(f, u, p, a, r)$

$$
\begin{aligned}
D_{(f, u, p, a, r)} J_{\epsilon}(F, U, P, A, R)= & \frac{1}{\epsilon}\left(\int_{\widetilde{\Omega}_{F}(T)} u(T) \cdot U(T) d x+\dot{a}(T) \cdot A(T)+r(T) R(T)\right) \\
& +\int_{0}^{T} \int_{\omega} \Theta f \cdot F .
\end{aligned}
$$


Next, we define the functional

$$
\begin{aligned}
L(f, u, p, a, r)= & \left(\partial_{t} u+(\tilde{u} \cdot \nabla) u-\operatorname{div} \sigma(u, p)-f 1_{\omega}, \operatorname{div} u, \ddot{a}-\frac{1}{m} \int_{\partial \widetilde{\Omega}_{S}(t)} \sigma(u, p) n,\right. \\
& \left.\dot{r}-\frac{1}{J} \int_{\partial \widetilde{\Omega}_{S}(t)}(\sigma(u, p) n) \cdot(x-\tilde{a})^{\perp}, \int_{0}^{T} \int_{\omega} \Theta f \cdot v_{1}, \int_{0}^{T} \int_{\omega} \Theta f \cdot v_{2}\right) .
\end{aligned}
$$

The constraints on $(f, u, p, a, r)$ can be expressed by the following equality

$$
L(f, u, p, a, r)=\left(\mathcal{O}, \mathcal{O}, \vec{O}_{\mathbb{R}^{2}}, 0, m_{1}\left(a_{0}, a_{1}, r_{0}, u_{0}\right), m_{2}\left(a_{1}, \theta_{0}, r_{0}, u_{0}\right)\right)
$$

where $\mathcal{O}$ is the null function defined in $L^{2}\left(0, T ; L^{2}\left(\widetilde{\Omega}_{F}(t)\right)\right)$. According to Lagrange's principle, there exist dual variables $\left(\hat{u}_{\epsilon}, \hat{p}_{\epsilon}, \hat{a}_{\epsilon}, \hat{r}_{\epsilon}\right), \hat{\alpha}_{\epsilon} \in \mathbb{R}$ and $\hat{\beta}_{\epsilon} \in \mathbb{R}$ such that

$$
\hat{u}_{\epsilon}=0 \text { on } \partial \Omega, \hat{u}_{\epsilon}=\dot{\hat{a}}_{\epsilon}+\hat{r}_{\epsilon}(x-\tilde{a})^{\perp} \text { on } \partial \widetilde{\Omega}_{S}(t)
$$

and for all $(F, U, P, A, R)$ such that $U=0$ on $\partial \Omega$ and $U=\dot{A}+R(x-\tilde{a})^{\perp}$ on $\partial \widetilde{\Omega}_{S}(t)$,

$$
D_{\left(f_{\epsilon}, u_{\epsilon}, p_{\epsilon}, a_{\epsilon}, r_{\epsilon}\right)} J_{\epsilon}(F, U, P, A, R)+\left\langle\left(\hat{u}_{\epsilon}, \hat{p}_{\epsilon}, \dot{\hat{a}}_{\epsilon}, \hat{r}_{\epsilon}, \hat{\alpha}_{\epsilon}, \hat{\beta}_{\epsilon}\right), L(F, U, P, A, R)\right\rangle=0
$$

Thus, we obtain that $\left(\hat{u}_{\epsilon}, \hat{p}_{\epsilon}, \hat{a}_{\epsilon}, \hat{r}_{\epsilon}\right)$ satisfies

$$
\left\{\begin{array}{l}
-\partial_{t} \hat{u}_{\epsilon}-(\tilde{u} \cdot \nabla) \hat{u}_{\epsilon}-\operatorname{div} \sigma\left(\hat{u}_{\epsilon}, \hat{p}_{\epsilon}\right)=0 \text { in } \widetilde{\Omega}_{F}(t), \\
m \ddot{\hat{a}}_{\epsilon}=-\int_{\partial \widetilde{\Omega}_{S}(t)} \sigma\left(\hat{u}_{\epsilon}, \hat{p}_{\epsilon}\right) n \\
J \dot{\hat{r}}_{\epsilon}=-\int_{\partial \widetilde{\Omega}_{S}(t)}\left(\sigma\left(\hat{u}_{\epsilon}, \hat{p}_{\epsilon}\right) n\right) \cdot(x-\tilde{a})^{\perp}, \\
\Theta f_{\epsilon}=\hat{u}_{\epsilon}-\hat{\alpha}_{\epsilon} v_{1}-\hat{\beta}_{\epsilon} v_{2} \text { in } \omega \\
\operatorname{div} \hat{u}_{\epsilon}=0 \text { in } \widetilde{\Omega}_{F}(t) \\
\hat{u}_{\epsilon}=0 \text { on } \partial \Omega \\
\hat{u}_{\epsilon}=\dot{\hat{a}}_{\epsilon}+\hat{r}_{\epsilon}(x-\tilde{a})^{\perp} \text { on } \partial \widetilde{\Omega}_{S}(t), \\
\hat{u}_{\epsilon}(T)=-\frac{1}{\epsilon} u_{\epsilon}(T) \text { in } \widetilde{\Omega}_{F}(T), \dot{\hat{a}}_{\epsilon}(T)=-\frac{1}{\epsilon} \dot{a}_{\epsilon}(T), \hat{r}_{\epsilon}(T)=-\frac{1}{\epsilon} r_{\epsilon}(T) .
\end{array}\right.
$$

Moreover, we can always suppose that $\hat{a}_{\epsilon}(T)=0$. Multiplying the first equation of this system by $u_{\epsilon}$, we obtain that

$\int_{0}^{T} \int_{\omega} f_{\epsilon} \cdot \hat{u}_{\epsilon}+\frac{1}{\epsilon} \int_{\widetilde{\Omega}_{F}(T)}\left|u_{\epsilon}(T)\right|^{2}+\frac{m}{\epsilon}\left|\dot{a}_{\epsilon}(T)\right|^{2}+\frac{J}{\epsilon}\left|r_{\epsilon}(T)\right|^{2}=-\int_{\Omega_{F}(0)} u_{0} \cdot \hat{u}_{\epsilon}(0)-m a_{1} \cdot \dot{\hat{a}}_{\epsilon}(0)-J r_{0} \hat{r}_{\epsilon}(0)$. 
Thus, we have

$$
\begin{aligned}
& \int_{0}^{T} \int_{\omega} \Theta\left|f_{\epsilon}\right|^{2}+\frac{1}{\epsilon} \int_{\widetilde{\Omega}_{F}(T)}\left|u_{\epsilon}(T)\right|^{2}+\frac{m}{\epsilon}\left|\dot{a}_{\epsilon}(T)\right|^{2}+\frac{J}{\epsilon}\left|r_{\epsilon}(T)\right|^{2} \\
& \leq-\int_{\Omega_{F}(0)} u_{0} \cdot \hat{u}_{\epsilon}(0)-m a_{1} \cdot \dot{\hat{a}}_{\epsilon}(0)-J r_{0} \hat{r}_{\epsilon}(0)-\hat{\alpha}_{\epsilon} \int_{0}^{T} \int_{\omega} f_{\epsilon} \cdot v_{1}-\hat{\beta}_{\epsilon} \int_{0}^{T} \int_{\omega} f_{\epsilon} \cdot v_{2} \\
& \leq C_{0}\left(\left\|\hat{u}_{\epsilon}(0)\right\|_{L^{2}\left(\Omega_{F}(0)\right)}+\left|\dot{\hat{a}}_{\epsilon}(0)\right|+\left|\hat{r}_{\epsilon}(0)\right|+\left|\hat{\alpha}_{\epsilon}\right|+\left|\hat{\beta}_{\epsilon}\right|\right),
\end{aligned}
$$

since $f_{\epsilon}$ satisfies (3.5). Here and in the following of this subsection, the constant $C_{0}$ linearly depends on $\left|a_{0}\right|$, $\left|a_{1}\right|,\left|\theta_{0}\right|,\left|r_{0}\right|$ and $\left\|u_{0}\right\|_{L^{2}\left(\Omega_{F}(0)\right)^{2}}$. From the observability inequality (3.8), we deduce that

$$
\begin{aligned}
& \left\|\hat{u}_{\epsilon}(0)\right\|_{L^{2}\left(\Omega_{F}(0)\right)}+\left|\dot{\hat{a}}_{\epsilon}(0)\right|+\left|\hat{r}_{\epsilon}(0)\right|+\left|\hat{\alpha}_{\epsilon}\right|+\left|\hat{\beta}_{\epsilon}\right| \\
& \leq C\left(\int_{0}^{T} \int_{\omega} \Theta^{-1}\left|\hat{u}_{\epsilon}-P\left(\hat{u}_{\epsilon}\right)\right|^{2}\right)^{1 / 2}+\left|\hat{\alpha}_{\epsilon}-P_{1}\left(\hat{u}_{\epsilon}\right)\right|+\left|\hat{\beta}_{\epsilon}-P_{2}\left(\hat{u}_{\epsilon}\right)\right| \\
& \leq C\left(\left(\int_{0}^{T} \int_{\omega} \Theta\left|f_{\epsilon}\right|^{2}\right)^{1 / 2}+\left|\hat{\alpha}_{\epsilon}-P_{1}\left(\hat{u}_{\epsilon}\right)\right|+\left|\hat{\beta}_{\epsilon}-P_{2}\left(\hat{u}_{\epsilon}\right)\right|\right),
\end{aligned}
$$

where $C$ depends on $T, \alpha$ and $R$. Since $f_{\epsilon}$ satisfies $(3.5)$ and $P\left(\hat{u}_{\epsilon}\right)$ satisfies $(3.7)$, we have

$$
\begin{gathered}
\int_{0}^{T} \int_{\omega} \Theta^{-1}\left(\left(P_{1}\left(\hat{u}_{\epsilon}\right)-\hat{\alpha}_{\epsilon}\right) v_{1}+\left(P_{2}\left(\hat{u}_{\epsilon}\right)-\hat{\beta}_{\epsilon}\right) v_{2}\right) \cdot v_{1}=m_{1}\left(a_{0}, a_{1}, r_{0}, u_{0}\right) \\
\int_{0}^{T} \int_{\omega} \Theta^{-1}\left(\left(P_{1}\left(\hat{u}_{\epsilon}\right)-\hat{\alpha}_{\epsilon}\right) v_{1}+\left(P_{2}\left(\hat{u}_{\epsilon}\right)-\hat{\beta}_{\epsilon}\right) v_{2}\right) \cdot v_{2}=m_{2}\left(a_{1}, \theta_{0}, r_{0}, u_{0}\right) .
\end{gathered}
$$

This allows to obtain the following estimate

$$
\left|\hat{\alpha}_{\epsilon}-P_{1}\left(\hat{u}_{\epsilon}\right)\right|+\left|\hat{\beta}_{\epsilon}-P_{2}\left(\hat{u}_{\epsilon}\right)\right| \leq C_{0}
$$

Thus, from (3.10), we obtain

$$
\int_{0}^{T} \int_{\omega} \Theta\left|f_{\epsilon}\right|^{2}+\frac{1}{\epsilon} \int_{\widetilde{\Omega}_{F}(T)}\left|u_{\epsilon}(T)\right|^{2}+\frac{m}{\epsilon}\left|\dot{a}_{\epsilon}(T)\right|^{2}+\frac{J}{\epsilon}\left|r_{\epsilon}(T)\right|^{2} \leq C C_{0}^{2},
$$

where $C$ depends on $T, \alpha$ and $R$. Moreover, according to the observability inequality (3.8), we also have

$$
\begin{aligned}
\int_{0}^{T} \int_{\widetilde{\Omega}_{F}(t)} \mathrm{e}^{-2 s \mathcal{V}}\left(\frac{1}{\varphi}\left|\partial_{t} \hat{u}_{\epsilon}\right|^{2}+\varphi^{3}\left|\hat{u}_{\epsilon}\right|^{2}\right)+\left|P_{1}\left(\hat{u}_{\epsilon}\right)\right|^{2}+\left|P_{2}\left(\hat{u}_{\epsilon}\right)\right|^{2} & \leq C \int_{0}^{T} \int_{\omega} \Theta^{-1}\left|\hat{u}_{\epsilon}-P\left(\hat{u}_{\epsilon}\right)\right|^{2} \\
& \leq C \int_{0}^{T} \int_{\omega} \Theta\left|f_{\epsilon}\right|^{2}+C C_{0}^{2}
\end{aligned}
$$

Thus, we get,

$$
\int_{0}^{T} \int_{\omega} \mathrm{e}^{-4 s \mathcal{V}^{*}+8 s \hat{\mathcal{V}}-2 s \mathcal{V}} \hat{\varphi}^{-20} \varphi^{-1}\left|\partial_{t} f_{\epsilon}\right|^{2} \leq C\left(\left|a_{0}\right|^{2}+\left|a_{1}\right|^{2}+\left|\theta_{0}\right|^{2}+\left|r_{0}\right|^{2}+\left\|u_{0}\right\|_{L^{2}\left(\Omega_{F}(0)\right)^{2}}^{2}\right),
$$

and

$$
\left\|f_{\epsilon}\right\|_{H^{1}\left(0, T ; L^{2}(\omega)\right)^{2}} \leq C\left(\left|a_{0}\right|+\left|a_{1}\right|+\left|\theta_{0}\right|+\left|r_{0}\right|+\left\|u_{0}\right\|_{L^{2}\left(\Omega_{F}(0)\right)^{2}}\right),
$$


where $C$ is a constant which depends on $T, \alpha$ and $R$. In particular, there exists a function $f \in H^{1}\left(0, T ; L^{2}(\omega)\right)^{2}$ such that, for a subsequence of $\left(f_{\epsilon}\right)$,

$$
f_{\epsilon} \rightarrow f \text { in } H^{1}\left(0, T ; L^{2}(\omega)\right)^{2} .
$$

Thanks to Proposition 2, $\left(u_{\epsilon}\right)$ weakly converges to $u$ in $L^{2}\left(0, T ; H^{2}\left(\widetilde{\Omega}_{F}(t)\right)\right)^{2} \cap H^{1}\left(0, T ; L^{2}\left(\widetilde{\Omega}_{F}(t)\right)\right)^{2},\left(p_{\epsilon}\right)$ weakly converges to $p$ in $L^{2}\left(0, T ; H^{1}\left(\widetilde{\Omega}_{F}(t)\right)\right),\left(a_{\epsilon}\right)$ weakly converges to $a$ in $H^{2}(0, T)^{2}$ and $\left(r_{\epsilon}\right)$ weakly converges to $r$ in $H^{1}(0, T)$ where $(u, p, a, r)$ is the solution of (1.21) together with the control $f$. Moreover, by passing to the limit in (3.11), we get

$$
u(T, \cdot)=0 \text { in } \widetilde{\Omega}_{F}(T), \dot{a}(T)=0, r(T)=0 .
$$

At last, since $f$ satisfies (3.5), we also have that $a(T)=0$ and $\theta(T)=0$. Thus, we have proved Theorem 2 .

Proposition 6. We suppose that $u_{0}, a_{0}, a_{1}, \theta_{0}$ and $r_{0}$ satisfy (1.13) and we consider an initial structure domain $\Omega_{S}(0)$ such that (1.9) is satisfied. We consider $\tilde{a} \in H^{2}(0, T)^{2}, \tilde{r} \in H^{1}(0, T)$ which satisfy (1.17) for some $\alpha>0$ and $\tilde{u}$ which satisfies conditions (1.18) to (1.20) and such that

$$
\|\tilde{a}\|_{H^{2}(0, T)^{2}}+\|\tilde{r}\|_{H^{1}(0, T)}+\|\tilde{u}\|_{L^{\infty}\left(0, T ; L^{\infty}\left(\tilde{\Omega}_{F}(t)\right)\right)^{2}}+\|\tilde{u}\|_{W^{1,4}\left(0, T ; L^{4}\left(\tilde{\Omega}_{F}(t)\right)\right)^{2}} \leq R .
$$

Then, our system is null controllable in the sense of Definition 2. Moreover, the control $f$ belongs to $H^{1}\left(0, T ; L^{2}(\omega)\right)^{2}$ satisfies $f(t=0)=0$ and

$$
\|f\|_{H^{1}\left(0, T ; L^{2}(\omega)\right)^{2}} \leq C_{3}\left(\left|a_{0}\right|+\left|a_{1}\right|+\left|\theta_{0}\right|+\left|r_{0}\right|+\left\|u_{0}\right\|_{L^{2}\left(\Omega_{F}(0)\right)^{2}}\right),
$$

where $C_{3}$ is a constant which depends on $T, \alpha$ and $R$ and the solution $(u, p, a, r)$ has the following regularity:

$$
\begin{aligned}
& u \in H^{1}\left(0, T ; H^{2}\left(\widetilde{\Omega}_{F}(t)\right)\right)^{2} \cap H^{2}\left(0, T ; L^{2}\left(\widetilde{\Omega}_{F}(t)\right)\right)^{2} \cap W^{1, \infty}\left(0, T ; H^{1}\left(\widetilde{\Omega}_{F}(t)\right)\right)^{2}, \\
& p \in H^{1}\left(0, T ; H^{1}\left(\widetilde{\Omega}_{F}(t)\right)\right), a \in H^{3}(0, T)^{2}, r \in H^{2}(0, T),
\end{aligned}
$$

and the norms of $(u, p, a, r)$ in these spaces are bounded by

$$
\|f\|_{H^{1}\left(0, T ; L^{2}(\omega)\right)^{2}}+\left\|u_{0}\right\|_{H^{3}\left(\Omega_{F}(0)\right)^{2}}+\left|a_{0}\right|+\left|a_{1}\right|+\left|\theta_{0}\right|+\left|r_{0}\right| .
$$

Proof of Proposition 6. The properties satisfied by $\Theta$ imply that $f_{\epsilon}(t=0)=0$ and thus

$$
f(t=0)=0 \text { in } \omega .
$$

Then, the proposition results from the regularity result given by Proposition 3.

\section{LOCAL NULL CONTROLLABiLity}

We are now able to prove Theorem 1. We will prove this theorem using a fixed point argument. Formally, we want to prove that the application which maps $(\tilde{u}, \tilde{a}, \tilde{r})$ on $(u, a, r)$ the controlled solution given by Proposition 6 admits a fixed point if initial conditions are small enough. This fixed point will be the controlled solution of the nonlinear problem. But the space where $(\tilde{u}, \tilde{a}, \tilde{r})$ is given depends on $\tilde{a}$ and $\tilde{r}$ themselves; indeed $(\tilde{u}, \tilde{a}, \tilde{r})$ has to satisfy conditions (1.18) and (1.19) where the spaces $\widetilde{\Omega}_{S}(t)$ and $\widetilde{\Omega}_{F}(t)$ are given by $\tilde{a}$ and $\tilde{r}$. Thus, we are not able to find a fixed point on this kind of spaces. Consequently, we will first construct $(\tilde{u}, \tilde{a}, \tilde{r})$ from uncoupled velocities $(\tilde{u}, \tilde{a}, \tilde{r})$ given on the initial domains.

We define

According to (1.9), $\alpha_{0}>0$.

$$
\alpha_{0}=\frac{1}{2} \mathrm{~d}\left(\overline{\Omega_{S}(0)}, \partial(\Omega \backslash \omega)\right) .
$$


Lemma 7. If $\tilde{a}$ and $\tilde{\theta}$ satisfy

$$
\left\|\tilde{a}-a_{0}\right\|_{L^{\infty}(0, T)^{2}} \leq \frac{\alpha_{0}}{2},\left\|R_{\tilde{\theta}-\theta_{0}}-I d\right\|_{L^{\infty}\left(0, T ; \mathcal{M}_{2 \times 2}(\mathcal{R})\right)} \leq \frac{\alpha_{0}}{2}\left(\sup _{y \in \Omega_{S}(0)}\left|y-a_{0}\right|\right)^{-1},
$$

then

$$
d\left(\widetilde{\widetilde{\Omega}_{S}(t)}, \partial(\Omega \backslash \omega)\right) \geq \alpha_{0}
$$

Proof of Lemma 7. We have

$$
\mathrm{d}\left(\widetilde{\widetilde{\Omega}_{S}(t)}, \partial(\Omega \backslash \omega)\right)=\inf _{x \in \tilde{\Omega}_{S}(t), z \in \partial(\Omega \backslash \omega)}|x-z|=\inf _{y \in \Omega_{S}(0), z \in \partial(\Omega \backslash \omega)}\left|\tilde{a}(t)+R_{\tilde{\theta}(t)-\theta_{0}}\left(y-a_{0}\right)-z\right|,
$$

and, for all $y \in \Omega_{S}(0), z \in \partial(\Omega \backslash \omega)$,

$$
\begin{aligned}
\left|\tilde{a}(t)+R_{\tilde{\theta}(t)-\theta_{0}}\left(y-a_{0}\right)-z\right| & \geq|y-z|-\left|\tilde{a}(t)+R_{\tilde{\theta}(t)-\theta_{0}}\left(y-a_{0}\right)-y\right| \\
& \geq \mathrm{d}\left(\overline{\Omega_{S}(0)}, \partial(\Omega \backslash \omega)\right)-\left|\tilde{a}(t)+R_{\tilde{\theta}(t)-\theta_{0}}\left(y-a_{0}\right)-y\right| .
\end{aligned}
$$

Moreover, we have

$$
\left|\tilde{a}(t)+R_{\tilde{\theta}(t)-\theta_{0}}\left(y-a_{0}\right)-y\right| \leq\left|\tilde{a}(t)-a_{0}\right|+\left|\left(R_{\tilde{\theta}(t)-\theta_{0}}-I d\right)\left(y-a_{0}\right)\right| \leq \alpha_{0} .
$$

This allows to obtain Lemma 7.

We consider the following spaces

$$
\begin{aligned}
Y & =L^{\infty}\left((0, T) \times \Omega_{F}(0)\right)^{2} \cap W^{1,4}\left(0, T ; L^{4}\left(\Omega_{F}(0)\right)\right)^{2} \cap L^{\infty}\left(0, T ; H_{0}^{1}\left(\Omega_{F}(0)\right)\right)^{2} \\
Z & =\left\{(\tilde{w}, \tilde{a}, \tilde{r}) \in Y \times W^{2,4}(0, T)^{2} \times W^{1,4}(0, T) / \operatorname{div} \tilde{w}=0 \text { in } \Omega_{F}(0)\right\} .
\end{aligned}
$$

We consider $(\tilde{w}, \tilde{a}, \tilde{r}) \in Z$ and $\tilde{\theta}$ such that

$$
\tilde{a}(0)=a_{0}, \dot{\tilde{a}}(0)=a_{1}, \tilde{\theta}(0)=\theta_{0}, \tilde{r}(0)=r_{0},
$$

where $\tilde{\theta}$ is the angle associated to the angular velocity $\tilde{r}$. We define an odd and nondecreasing function $\phi \in \mathcal{C}^{2}(\mathbb{R})$ such that

$$
\phi(x)= \begin{cases}x & \text { in }[0,3 / 4], \\ 1 & \text { in }[1, \infty[,\end{cases}
$$

and we introduce the family of functions defined for $K>0, h \in \mathbb{R}$ by

$$
\mathcal{T}_{K, h}(x)=h+K \phi((x-h) / K), \forall x \in \mathbb{R} .
$$

Thus,

$$
\mathcal{T}_{K, h}(x)= \begin{cases}x & \text { if }|x-h| \leq 3 K / 4, \\ h+K & \text { if }|x-h| \geq K .\end{cases}
$$

Since, for all $x \in \mathbb{R},\left|\mathcal{T}_{K, h}(x)-h\right| \leq K$, we can find $b$ and $c$ (small enough) depending only on $\alpha_{0}$ and $\Omega_{S}(0)$ such that $\left(\mathcal{T}_{b, a_{0}^{1}}\left(\tilde{a}^{1}\right), \mathcal{T}_{b, a_{0}^{2}}\left(\tilde{a}^{2}\right)\right)$ and $\mathcal{T}_{c, \theta_{0}}(\widetilde{\theta})$ satisfy $(4.1)$ where $a_{0}=\left(a_{0}^{1}, a_{0}^{2}\right)$ and $\tilde{a}=\left(\tilde{a}^{1}, \tilde{a}^{2}\right)$. We denote $\mathcal{T}_{b, a_{0}}(\tilde{a})=\left(\mathcal{T}_{b, a_{0}^{1}}\left(\tilde{a}^{1}\right), \mathcal{T}_{b, a_{0}^{2}}\left(\tilde{a}^{2}\right)\right)$. Thus, we can extend the flow $\tilde{X}$ defined on $\Omega_{S}(0)$ by

$$
\widetilde{X}(t, y)=\mathcal{T}_{b, a_{0}}(\tilde{a})+R_{\mathcal{T}_{c, \theta_{0}}(\tilde{\theta})-\theta_{0}}\left(y-a_{0}\right)
$$


and its inverse $\widetilde{Y}$ as it is done in Lemma 3. The displacements $\mathcal{T}_{b, a_{0}}(\tilde{a}), \mathcal{T}_{c, \theta_{0}}(\widetilde{\theta})$ and the moving domains associated to $\widetilde{X}$ satisfy condition (1.17) with $\alpha=\alpha_{0}$. We denote $\tilde{u}_{S}$ the velocity associated to $\widetilde{X}$. Now, we define on $(0, T) \times \Omega$,

$$
\tilde{u}(t, x)=\tilde{u}_{S}(t, x)+\nabla \tilde{X}(t, \tilde{Y}(t, x)) \tilde{w}(t, \tilde{Y}(t, x)) .
$$

This velocity combined with the rigid motion $\mathcal{T}_{b, a_{0}}(\tilde{a})$ and $\mathcal{T}_{c, \theta_{0}}(\widetilde{\theta})$ satisfies (1.18) and (1.19). We denote $u_{S}^{0}$ the velocity which extends $a_{1}+r_{0}\left(x-a_{0}\right)^{\perp}$ thanks to Lemma 2 and we define

$$
w_{0}=u_{0}-u_{S}^{0} .
$$

We see that, if $\tilde{w}(t=0)=w_{0}$ and $(\tilde{a}, \tilde{r})$ satisfies (4.2), then $\tilde{u}$ satisfies (1.20). Next, we introduce $Z_{R}$ a subset of $Z$ by

$$
\begin{gathered}
Z_{R}=\left\{(\tilde{w}, \tilde{a}, \tilde{r}) \in Z /\|\tilde{w}\|_{Y}+\|\tilde{a}\|_{W^{2,4}(0, T)^{2}}+\|\tilde{r}\|_{W^{1,4}(0, T)} \leq R, \tilde{w}(t=0)=w_{0} \text { in } \Omega_{F}(0),\right. \\
\left.\tilde{a}(0)=a_{0}, \dot{\tilde{a}}(0)=a_{1}, \tilde{\theta}(0)=\theta_{0}, \tilde{r}(0)=r_{0}\right\}
\end{gathered}
$$

Let us take $(\tilde{w}, \tilde{a}, \tilde{r}) \in Z_{R}$. We can apply Proposition 6 which associates to $\left(\tilde{u}, \mathcal{T}_{b, a_{0}}(\tilde{a}), \mathcal{T}_{c, \theta_{0}}(\widetilde{\theta})\right)$ a control $f \in H^{1}\left(0, T ; L^{2}(\omega)\right)^{2}$ and an associated state $(u, p, a, r)$ solution of (1.21) such that

$$
u(T, \cdot)=0 \text { in } \Omega, a(T)=0, \theta(T)=0,
$$

and

$$
f(t=0)=0 \text { in } \omega,\|f\|_{H^{1}\left(0, T ; L^{2}(\omega)\right)^{2}} \leq C_{4}\left(\left|a_{0}\right|+\left|a_{1}\right|+\left|\theta_{0}\right|+\left|r_{0}\right|+\left\|u_{0}\right\|_{L^{2}\left(\Omega_{F}(0)\right)^{2}}\right),
$$

where $C_{4}$ depends on $T, \alpha_{0}$ and $R$. Indeed, since $(\tilde{w}, \tilde{a}, \tilde{r})$ belongs to $Z_{R}$,

$$
\|\tilde{a}\|_{H^{2}(0, T)^{2}}+\|\tilde{r}\|_{H^{1}(0, T)}+\|\tilde{u}\|_{L^{\infty}\left(0, T ; L^{\infty}\left(\widetilde{\Omega}_{F}(t)\right)\right)^{2}}+\|\tilde{u}\|_{W^{1,4}\left(0, T ; L^{4}\left(\widetilde{\Omega}_{F}(t)\right)\right)^{2}} \leq C R,
$$

where $C$ depends on $T$ and $\alpha_{0}$. by

According to proposition $6,(a, r)$ belongs to $H^{3}(0, T)^{2} \times H^{2}(0, T)$. We consider the velocity defined on $\widetilde{\Omega}_{S}(t)$

$$
\dot{a}+r(x-\tilde{a})^{\perp}
$$

and we extend this velocity on $\Omega$ by a velocity $u_{S}$ which has the same properties as $\tilde{u}_{S}$ given by Lemma 2 . Then, we define $w$ by

$$
w(t, y)=\nabla \widetilde{Y}(t, \widetilde{X}(t, y))\left(u-u_{S}\right)(t, \widetilde{X}(t, y)), \forall y \in \Omega_{F}(0) .
$$

The velocity $u_{S}$ belongs to $H^{2}\left(0, T ; \mathcal{C}^{2}(\Omega)\right)^{2}$ and

$$
\left\|u_{S}\right\|_{H^{2}\left(0, T ; \mathcal{C}^{2}(\Omega)\right)^{2}} \leq C\left(\|a\|_{H^{3}(0, T)^{2}}+\|r\|_{H^{2}(0, T)}\right) .
$$

We easily check that $(w, a, r)$ belongs to $Z$ according to Proposition 6 and

$$
\|w\|_{Y}+\|a\|_{W^{2,4}(0, T)^{2}}+\|r\|_{W^{1,4}(0, T)} \leq C_{5}\left(\left|a_{0}\right|+\left|a_{1}\right|+\left|\theta_{0}\right|+\left|r_{0}\right|+\left\|u_{0}\right\|_{H^{3}\left(\Omega_{F}(0)\right)^{2}}\right),
$$

where the constant $C_{5}$ depends on $T, \alpha_{0}$ and $R$. Moreover, we also have

$$
w(T, \cdot)=0 \text { in } \Omega_{F}(0), a(T)=0, \theta(T)=0, \dot{a}(T)=0, r(T)=0 .
$$

For $(\tilde{w}, \tilde{a}, \tilde{r}) \in Z_{R}$, let us define the set $\Lambda(\tilde{w}, \tilde{a}, \tilde{r})$ by

$\Lambda(\tilde{w}, \tilde{a}, \tilde{r})=\left\{(w, a, r) \in Z\right.$ satisfying (4.4), (4.5) with $f \in H^{1}\left(0, T ; L^{2}(\omega)\right)^{2}$ satisfying (4.3) $\}$, 
and let us consider the set-valued mapping $\Lambda: Z_{R} \mapsto Z$. We will apply Kakutani's theorem to this mapping. First of all, according to what precedes, $\Lambda(\tilde{w}, \tilde{a}, \tilde{r})$ is always a nonempty subset of $Z$. Moreover, it is easy to see that it is a closed convex subset of $Z$. Next, since the control $f$ belongs to $H^{1}\left(0, T ; L^{2}(\omega)\right)^{2}$, we can apply Proposition 3 and deduce that

$$
\begin{aligned}
& w \in H^{1}\left(0, T ; H^{2}\left(\Omega_{F}(0)\right)\right)^{2} \cap H^{2}\left(0, T ; L^{2}\left(\Omega_{F}(0)\right)\right)^{2} \cap W^{1, \infty}\left(0, T ; H^{1}\left(\Omega_{F}(0)\right)\right)^{2}, \\
& a \in H^{3}(0, T)^{2}, r \in H^{2}(0, T) .
\end{aligned}
$$

Consequently, for each $(\tilde{w}, \tilde{a}, \tilde{r}) \in Z_{R}, \Lambda(\tilde{w}, \tilde{a}, \tilde{r}) \hookrightarrow K$ where $K$ is a compact subset of $Z$. We also have to prove that $\Lambda$ is upper hemicontinuous in $Z$. This will be true if, for all $\nu \in \mathbb{R}$ and for all $(v, b, s) \in Z^{\prime}$

$$
B(\nu, v, b, s)=\left\{(\tilde{w}, \tilde{a}, \tilde{r}) \in Z / \sup _{(w, a, r) \in \Lambda(\tilde{w}, \tilde{a}, \tilde{r})}\langle(v, b, s),(w, a, r)\rangle \geq \nu\right\}
$$

is a closed subset of $Z$.

We consider a sequence $\left(\tilde{w}_{n}, \tilde{a}_{n}, \tilde{r}_{n}\right)$ of $B(\nu, v, b, s)$ such that

$$
\left(\tilde{w}_{n}, \tilde{a}_{n}, \tilde{r}_{n}\right) \rightarrow(\tilde{w}, \tilde{a}, \tilde{r}) \text { in } Z
$$

and we want to prove that $(\tilde{w}, \tilde{a}, \tilde{r})$ belongs to $B(\nu, v, b, s)$. Since $\Lambda(\tilde{w}, \tilde{a}, \tilde{r})$ is compact, for every $n \in \mathbb{N}$, there exists $\left(w_{n}, a_{n}, r_{n}\right) \in \Lambda\left(\tilde{w}_{n}, \tilde{a}_{n}, \tilde{r}_{n}\right)$ such that

$$
\sup _{(w, a, r) \in \Lambda\left(\tilde{w}_{n}, \tilde{a}_{n}, \tilde{r}_{n}\right)}\langle(v, b, s),(w, a, r)\rangle=\left\langle(v, b, s),\left(w_{n}, a_{n}, r_{n}\right)\right\rangle .
$$

This sequence $\left(w_{n}, a_{n}, r_{n}\right)$ satisfies (4.4) and belongs to the compact subset $K$. Thus, it strongly converges to a limit $(w, a, r)$ in $Z$. In the same way, the sequence of controls $\left(f_{n}\right)$ associated to $\left(w_{n}, a_{n}, r_{n}\right)$ is bounded in $H^{1}\left(0, T ; L^{2}(\omega)\right)^{2}$ and weakly converges to $f$ in $H^{1}\left(0, T ; L^{2}(\omega)\right)^{2}$. Now, since $(w, a, r)$ belongs to $\Lambda(\tilde{w}, \tilde{a}, \tilde{r})$, we obtain that

$$
\sup _{(w, a, r) \in \Lambda(\tilde{w}, \tilde{a}, \tilde{r})}\langle(v, b, s),(w, a, r)\rangle \geq\langle(v, b, s),(w, a, r)\rangle=\lim _{n \rightarrow \infty}\left\langle(v, b, s),\left(w_{n}, a_{n}, r_{n}\right)\right\rangle \geq \nu .
$$

This proves that $\Lambda$ is upper hemicontinuous.

At last, according to (4.4), if

$$
\left|a_{0}\right|+\left|a_{1}\right|+\left|\theta_{0}\right|+\left|r_{0}\right|+\left\|u_{0}\right\|_{H^{3}\left(\Omega_{F}(0)\right)^{2}} \leq \frac{R}{C_{5}}
$$

$(w, a, r)$ belongs to $Z_{R}$. Thus, we consider initial data which satisfy (4.6) and we can apply Kakutani's fixed point theorem to the set-valued mapping $\Lambda: Z_{R} \mapsto Z_{R}$. Therefore, if initial data satisfy (4.6), we have the existence of a solution $(w, a, r)$ associated to a control $f \in H^{1}\left(0, T ; L^{2}(\omega)\right)^{2}$ which satisfies (4.4). The associated velocity $u$ together with $a, r$ and the pressure $p$ is solution of a nonlinear system where the domains are given by the flow

From (4.4), we deduce

$$
X(t, y)=\mathcal{T}_{b, a_{0}}(a)+R_{\mathcal{T}_{c, \theta_{0}}(\theta)-\theta_{0}}\left(y-a_{0}\right)
$$

$$
\|a\|_{W^{1, \infty}(0, T)^{2}}+\|r\|_{L^{\infty}(0, T)} \leq C_{6}\left(\left|a_{0}\right|+\left|a_{1}\right|+\left|\theta_{0}\right|+\left|r_{0}\right|+\left\|u_{0}\right\|_{H^{3}\left(\Omega_{F}(0)\right)^{2}}\right),
$$

where $C_{6}$ depends on $T, \alpha_{0}$ and $R$. Thus, for initial conditions such that

$$
\left|a_{0}\right|+\left|a_{1}\right|+\left|\theta_{0}\right|+\left|r_{0}\right|+\left\|u_{0}\right\|_{H^{3}\left(\Omega_{F}(0)\right)^{2}} \leq \frac{3}{4 T C_{6}} \min (b, c),
$$


we have $\mathcal{T}_{b, a_{0}}(a)=a$ and $\mathcal{T}_{c, \theta_{0}}(\theta)=\theta$. It implies that $(u, p, a, r)$ is solution of the problem (1.1) to (1.6) and satisfies (1.14). Therefore, for $\left(a_{0}, a_{1}, \theta_{0}, r_{0}, u_{0}\right)$ satisfying (4.6) and (4.7), we obtain Theorem 1.

Acknowledgements. The authors want to thank Jean-Pierre Puel and Sergio Guerrero for very fruitful remarks and discussions. This collaboration was partially supported by ECOS-Conicyt grants CO1E02 and CO4E08.

The second author acknowledges FONDECYT 1030808 grant and the support of the CNRS for a three month stay at the Jacques-Louis Lions Laboratory, University Pierre et Marie Curie - Paris 6, France in 2005, since a part of this joint work was possible thanks to this stay.

\section{REFERENCES}

[1] S. Anita and V. Barbu, Null controllability of nonlinear convective heat equations. ESAIM: COCV 5 (2000) 157-173.

[2] M. Boulakia and A. Osses, Local null controllability of a two-dimensional fluid-structure interaction problem. Prépublication 139, UVSQ (octobre 2005).

[3] C. Conca, J. San Martin and M. Tucsnak, Existence of solutions for the equations modelling the motion of a rigid body in a viscous fluid. Comm. Partial Differential Equations 25 (2000) 1019-1042.

[4] J.M. Coron and S. Guerrero, Singular optimal control: A linear 1-D parabolic-hyperbolic example. Asymptot. Anal. 44 (2005) $237-257$.

[5] B. Desjardins and M.J. Esteban, Existence of weak solutions for the motion of rigid bodies in a viscous fluid. Arch. Ration. Mech. Anal. 146 (1999) 59-71.

[6] A. Doubova and E. Fernandez-Cara, Some control results for simplified one-dimensional models of fluid-solid interaction. Math. Models Methods Appl. Sci. 15 (2005) 783-824.

[7] C. Fabre and G. Lebeau, Prolongement unique des solutions de l'équation de Stokes. Comm. Partial Diff. Equations 21 (1996) 573-596.

[8] C. Fabre, J.-P. Puel and E. Zuazua, Approximate controllability of the semilinear heat equation. Proc. Royal Soc. Edinburgh 125A (1995) 31-61.

[9] E. Fernandez-Cara and E. Zuazua, The cost of approximate controllability for heat equations: the linear case. Adv. Differential Equations 5 (2000) 465-514.

[10] E. Fernandez-Cara, S. Guerrero, O. Yu. Imanuvilov and J.-P. Puel, Local exact controllability of the Navier-Stokes system. J. Math. Pures Appl. 83 (2004) 1501-1542.

[11] A.V. Fursikov and O.Yu. Imanuvilov, Controllability of Evolution Equations, Lecture Notes Series 34, Seoul National University, Research Institute of Mathematics, Global Analysis Research Center, Seoul (1996).

[12] O.Yu. Imanuvilov, Remarks on exact controllability for the Navier-Stokes equations. ESAIM: COCV 6 (2001) 39-72.

[13] O.Yu. Imanuvilov and J.-P. Puel, Global Carleman estimates for weak solutions of elliptic nonhomogeneous Dirichlet problems. Internat. Math. Res. Notices 16 (2003) 883-913.

[14] O.Yu. Imanuvilov and T. Takahashi, Exact controllability of a fluid-rigid body system. Prépublication IECN (novembre 2005).

[15] O. Nakoulima, Contrôlabilité à zéro avec contraintes sur le contrôle. C. R. Acad. Sci. Paris Ser. I 339 (2004) $405-410$.

[16] A. Osses and J.P. Puel, Approximate controllability for a linear model of fluid structure interaction. ESAIM: COCV 4 (1999) 497-513.

[17] J.P. Raymond and M. Vanninathan, Exact controllability in fluid-solid structure: the Helmholtz model. ESAIM: COCV 11 (2005) 180-203.

[18] J. San Martin, V. Starovoitov and M. Tucsnak, Global weak solutions for the two dimensional motion of several rigid bodies in an incompressible viscous fluid. Arch. Rational Mech. Anal. 161 (2002) 113-147.

[19] T. Takahashi, Analysis of strong solutions for the equations modeling the motion of a rigid-fluid system in a bounded domain, Adv. Differential Equations 8 (2003) 1499-1532.

[20] R. Temam, Behaviour at time $t=0$ of the solutions of semi-linear evolution equations. J. Diff. Equations 43 (1982) 73-92.

[21] J.L. Vázquez, E. Zuazua, Large time behavior for a simplified 1D model of fluid-solid interaction. Comm. Partial Differential Equations 28 (2003) 1705-1738.

[22] J.L. Vázquez and E. Zuazua, Lack of collision in a simplified 1-dimensional model for fluid-solid interaction. Math. Models Methods Apll. Sci., M3AS 16 (2006) 637-678.

[23] X. Zhang and E. Zuazua, Polynomial decay and control of a 1-d hyperbolic-parabolic coupled system. J. Differential Equations 204 (2004) 380-438. 